\title{
Herschel-PACS photometry of the five major moons of Uranus ${ }^{\star}$
}

\author{
Ö. H. Detre ${ }^{1}$, T. G. Müller ${ }^{2}$, U. Klaas ${ }^{1}$, G. Marton ${ }^{3,4}$, H. Linz ${ }^{1}$, and Z. Balog ${ }^{1,5}$ \\ ${ }^{1}$ Max-Planck-Institut für Astronomie (MPIA), Königstuhl 17, 69117 Heidelberg, Germany \\ e-mail: detre@mpia.de \\ 2 Max-Planck-Institut für extraterrestrische Physik (MPE), PO Box 1312, Giessenbachstraße, 85741 Garching, Germany \\ ${ }^{3}$ Konkoly Observatory, Research Centre for Astronomy and Earth Sciences, Konkoly Thege-Miklós 15-17, 1121 Budapest, \\ Hungary \\ ${ }^{4}$ ELTE Eötvös Loránd University, Institute of Physics, Pázmány Péter 1/A, 1171 Budapest, Hungary \\ 5 Astronomisches Recheninstitut des Zentrums für Astronomie, Mönchhofstrasse 12-14, 69120 Heidelberg, Germany
}

Received 30 January 2020 / Accepted 9 June 2020

\begin{abstract}
Aims. We aim to determine far-infrared fluxes at 70,100, and $160 \mu \mathrm{m}$ for the five major Uranus satellites, Titania, Oberon, Umbriel, Ariel, and Miranda. Our study is based on the available calibration observations at wavelengths taken with the PACS photometer aboard the Herschel Space Observatory.

Methods. The bright image of Uranus was subtracted using a scaled Uranus point spread function (PSF) reference established from all maps of each wavelength in an iterative process removing the superimposed moons. The photometry of the satellites was performed using PSF photometry. Thermophysical models of the icy moons were fitted to the photometry of each measurement epoch and auxiliary data at shorter wavelengths.

Results. The best-fit thermophysical models provide constraints for important properties of the moons, such as surface roughness and thermal inertia. We present the first thermal infrared radiometry longward of $50 \mu \mathrm{m}$ for the four largest Uranian moons, Titania, Oberon, Umbriel, and Ariel, at epochs with equator-on illumination. Due to this inclination geometry, heat transport took place to the night side so that thermal inertia played a role, allowing us to constrain that parameter. Also, we found some indication for differences in the thermal properties of leading and trailing hemispheres. The total combined flux contribution of the four major moons relative to Uranus is $5.7 \times 10^{-3}, 4.8 \times 10^{-3}$, and $3.4 \times 10^{-3}$ at 70,100 , and $160 \mu \mathrm{m}$, respectively. We therefore precisely specify the systematic error of the Uranus flux by its moons when Uranus is used as a far-infrared prime flux calibrator. Miranda is considerably fainter and always close to Uranus, impeding reliable photometry.

Conclusions. We successfully demonstrate an image processing technique for PACS photometer data that allows us to remove a bright central source and reconstruct point source fluxes on the order of $10^{-3}$ of the central source as close as $\approx 3 \times$ the half width at half maximum of the PSF. We established improved thermophysical models of the five major Uranus satellites. Our derived thermal inertia values resemble those of trans-neptunian object (TNO) dwarf planets, Pluto and Haumea, more than those of smaller TNOs and Centaurs at heliocentric distances of about $30 \mathrm{AU}$.
\end{abstract}

Key words. space vehicles: instruments - techniques: image processing - techniques: photometric - infrared: planetary systems radiation mechanisms: thermal

\section{Introduction}

The planet Uranus is a well suited primary flux standard at the upper end of the accessible flux range for a number of contemporary far-infrared space and airborne photometers, such as ISOPHOT (Lemke et al. 1996), Herschel-PACS (Poglitsch et al. 2010), and HAWC+ (Harper et al. 2018). Uranus is also an important flux/amplitude calibrator for submm/mm/cm ground-based observatories, such as IRAM (Kramer et al. 2008) or JCMT (SCUBA-2; Chapin et al. 2013).

Uranus was routinely observed during the Herschel mission (Pilbratt et al. 2010) as part of the PACS photometer 70, 100 , and $160 \mu \mathrm{m}$ filter flux calibration program, in particular for a quantitative verification of the flux non-linearity correction for PACS (Müller et al. 2016).

Due to its flux density of $>500 \mathrm{Jy}$, Uranus exhibits an extended intensity profile in the PACS maps which reaches out to radii $>1^{\prime}$, and overwhelms the emission from its moons. An

\footnotetext{
${ }^{\star}$ Herschel is an ESA space observatory with science instruments provided by European-led Principal Investigator consortia and with important participation from NASA.
}

example is the Uranus image shown in the left panel of Fig. 1. Nevertheless, with a detailed comparison of the Uranus image with a PACS reference point spread function (PSF; Fig. 1 middle), it is possible to trace extra features on top of the Uranus PSF. That is how we recognised the two largest and most distant of the five major Uranian moons, Titania and Oberon, in the PACS maps. Titania and Oberon were discovered by the name patron of the Herschel Space Observatory, William (Wilhelm) Herschel, himself. In the following sections, we describe the method used to generate the Uranus reference PSF and subtract it from the maps in order to extract FIR fluxes for all five major moons of Uranus. This photometry will be compared with the thermophysical modelling of the moons.

\section{Data reduction}

\subsection{Input maps for Uranus PSF reference}

The key to a good PSF subtraction is to have a good reference PSF. Since Uranus is a slightly extended source $\left(\approx 3^{\prime \prime} .5\right)$, the standard PACS PSF references based on maps of the asteroids Ceres 

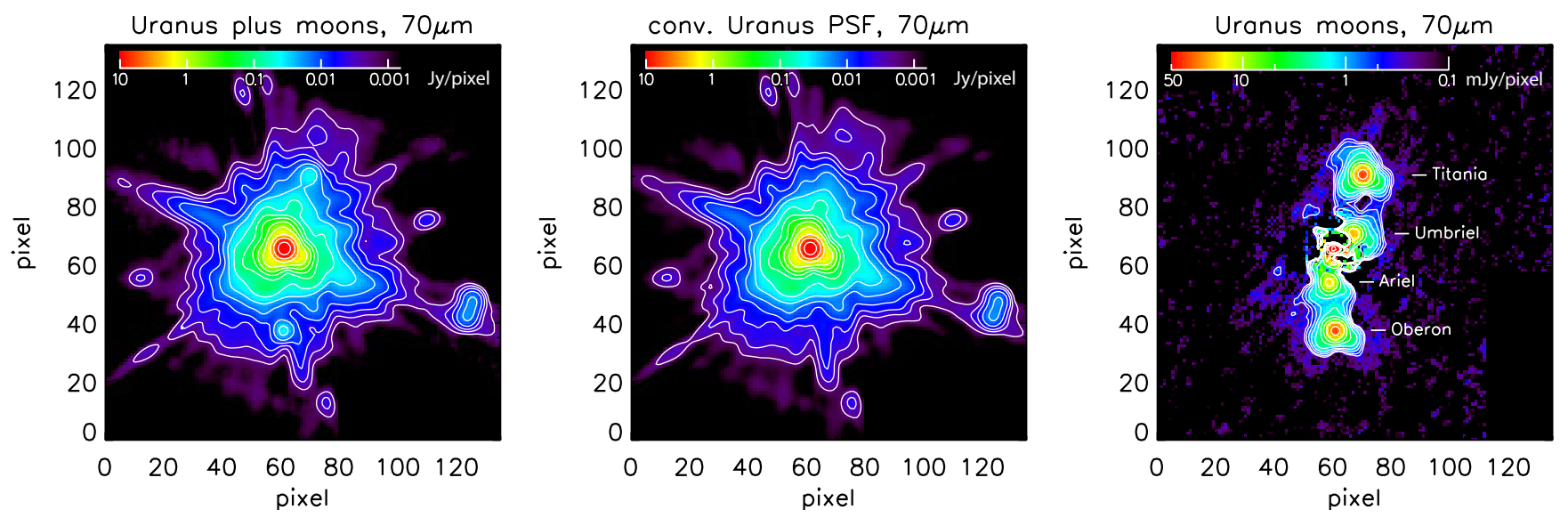

Fig. 1. PACS $70 \mu \mathrm{m}$ scan map of Uranus (OBSIDs 1342223982+83) from OD 789 (2011-07-12T01:21:57). Pixel scale is 1'. 1. Left: original map generated by high-pass filtering and co-addition of scan and cross-scan containing Uranus plus its moons. This map was actually generated as an average of the nine different map parameter data sets per scan direction Middle: convolved Uranus PSF for OD 789 generated from the Uranus reference PSF by PSF matching, cf. Sect. 2.3. It shows a number of pronounced PSF features, while the absence of Titania and Oberon is clearly visible. Right: residual map after subtraction of the convolved Uranus PSF map from the original map. The four Uranus moons Titania, Umbriel, Ariel, and Oberon become clearly visible. For better visualisation different flux scales were used for the individual images.

and Vesta (Lutz 2015) ${ }^{1}$ did not provide adequate PSF subtraction results. We therefore decided to construct a Uranus reference PSF (Ref PSF from now on) out of the individual Uranus maps in each PACS filter.

The Herschel Science Archive contains twenty individual scan map measurements of Uranus, taken over the entire course of the mission at five distinct epochs (cf. Table A.1). Within each of those five epochs, four scan map observations were taken approximately $6 \mathrm{~min}$ apart from each other. The PACS photometer was able to take data simultaneously in the $160 \mu \mathrm{m}$ filter as well as either the 70 or $100 \mu \mathrm{m}$ filter. The starting point of our PSF analysis were, therefore, the ten 70 and $100 \mu \mathrm{m}$ and the twenty $160 \mu \mathrm{m}$, high-pass filtered and flux-calibrated level 2 scan maps produced for the Uranus photometry as published in Müller et al. (2016). The data reduction and calibration performed in HIPE $^{2}$ (Ott 2010) up to this level is described in Balog et al. (2014). A general description of PACS high-pass filter processing is given in the PACS Handbook (Exter et al. 2018). In order to determine any dependence of our PSF photometry on the data reduction, we re-processed the maps with a variety of map parameter combinations for HPF radius and pixfrac, as listed in Table 1. The variation of the results among the nine different created maps of the same observation identifier (OBSID) is one component in our photometric uncertainty assessment. The related uncertainty is listed under $\sigma_{\text {red }}$ in Tables A.1-A.6.

\subsection{Establishment of the Uranus reference PSFS}

As a first step the WCS (world coordinate system) astrometries of the images were corrected by finding the centre of Uranus. This was crucial to correct the majority of astrometric uncertainties of the images. In addition to the standard flux calibration in HIPE a final flux calibration step was done by removing the dependence of the detector response on the telescope background, a calibration feature which is described in Balog et al. (2014).

\footnotetext{
1 https://www.cosmos.esa.int/documents/12133/996891/ PACS+photometer+point+spread+function, Fig. 7.

2 HIPE is a joint development by the Herschel Science Ground Segment Consortium, consisting of ESA, the NASA Herschel Science Center, and the HIFI, PACS and SPIRE consortia.
}

Table 1. Applied scan map parameters for the input maps of the PSF fitting step.

\begin{tabular}{rcccc}
\hline \hline $\begin{array}{r}\text { Filter } \\
(\mu \mathrm{m})\end{array}$ & $\begin{array}{c}F W H M_{\mathrm{PSF}} \\
\left({ }^{\prime \prime}\right)\end{array}$ & $\begin{array}{c}\text { outpix } \\
\left({ }^{\prime \prime}\right)\end{array}$ & HPF radius ${ }^{(a)}$ & pixfrac \\
\hline 70 & 5.6 & 1.1 & $15,20,35$ & $0.1,0.5,1.0$ \\
100 & 6.8 & 1.4 & $15,20,35$ & $0.1,0.5,1.0$ \\
160 & 10.7 & 2.1 & $30,40,70$ & $0.1,0.5,1.0$ \\
\hline
\end{tabular}

Notes. $F W H M_{\mathrm{PSF}}$ is the average full width at half maximum of the PSF for a point-like source in the corresponding filter. "Outpix" marks the output pixel size in the final map. This was kept constant, which means a sampling of the PSF FWHM by 5 pixels in each filter. "HPF" is the abbreviation for the high-pass filter, "pixfrac" is the ratio of drop size to input pixel size used for the drizzling algorithm (Fruchter \& Hook 2002) within the photProject() mapper. ${ }^{(a)}$ This parameter determines the elementary section of a scan over which the high-pass filter algorithm computes a running median value. Its unit is "number of read-outs". The spatial interval between two readouts is $\alpha_{\mathrm{ro}}=\frac{v_{\mathrm{scan}}}{\gamma_{\mathrm{re}}}$. For the standard $v_{\mathrm{ro}}=10 \mathrm{~Hz}$ read-out scheme in PACS prime mode, and a scan speed $v_{\text {scan }}=20 " \mathrm{~s}^{-1}$, the spatial interval $\alpha_{\text {ro }}$ between two read-outs corresponds to $2 "$. The entire width of the HPF window (") $=[(2 \times$ HPF radius) +1$] \times \alpha_{\text {ro }}$.

The relation of detector responsivity with telescope background could be established from the Uranus observations themselves with a very high signal-to-noise-ratio $(\mathrm{S} / \mathrm{N})$. All images were then flux-normalised to a mean Uranus-to-Herschel distance and rotated to the same reference angle. The distance correction was on the order of $6 \%$, while the detector response correction with telescope background was on the order of $1 \%$. Details of these flux corrections are detailed in Appendix B. Following these corrections, the uncertainty of Uranus flux was within a remarkable $0.19-0.27 \%$ depending on the filter, proving the outstanding flux stability of the PACS instrument. This was important because flux variation could have a negative effect on the creation of the median image for the Ref PSF in the next steps. On the other hand, an arbitrary normalisation compensating for the flux differences would render any later photometry unreliable. 


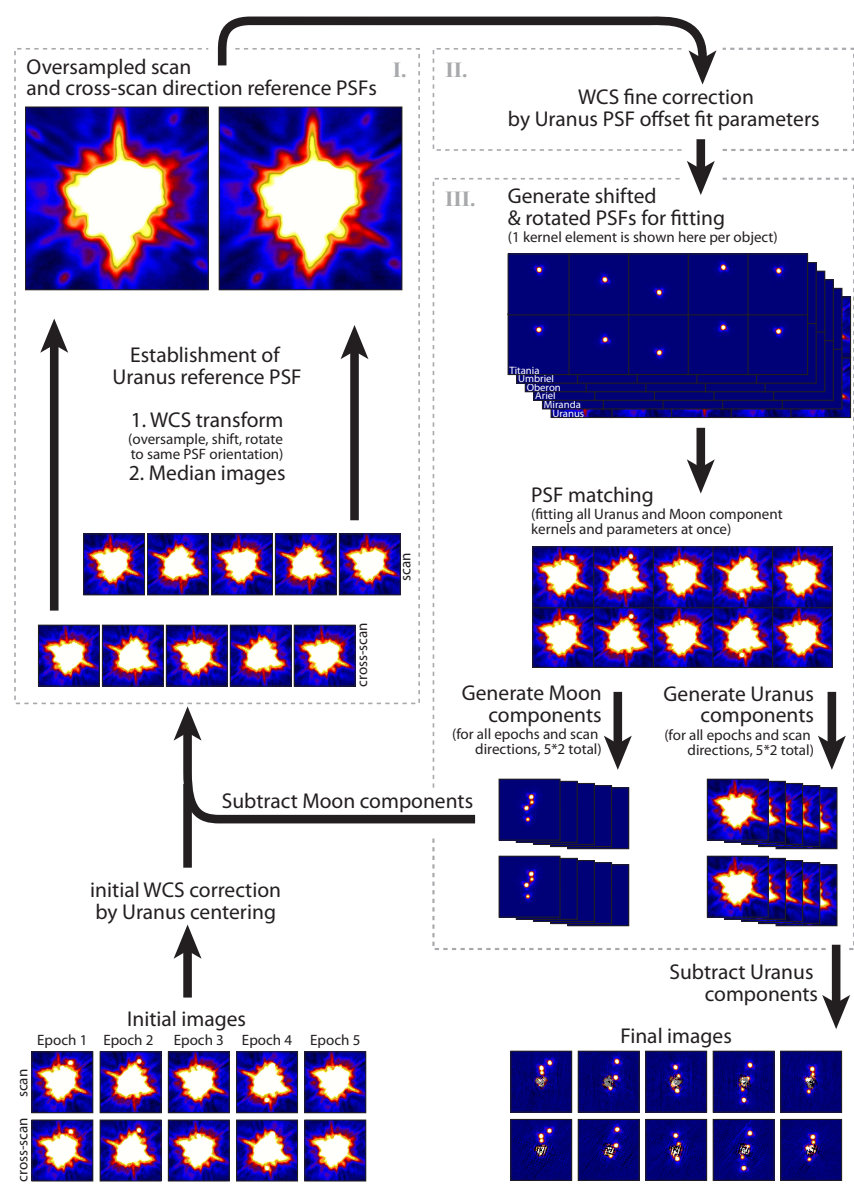

Fig. 2. Flowchart for the iteration cycle. The dashed boxes show the three main parts of the iteration loop. The calculation starts at bottom left by initial WCS correction of the raw images. The iteration cycle is stopped when the fit parameters do not change significantly. The 25 iteration cycles were needed for each dataset with different HPF and pixfrac values and, of course, each filter. Finally, Uranus-subtracted images are at the bottom right.

Four-time oversampling was used for the Ref PSF (FWHM was sampled by 20 pixels) to mitigate the information loss by the re-sampling of the data back and forth. A separate Ref PSF was generated for each of the two scan directions due to minor differences between them. The very first Ref PSF was generated by a simple median over the individual images on each pixel. The median removed the orbiting moons for most of the pixels around the PSF centre. However, for some areas of the Ref PSF the moons were overlapping multiple times. To remove the remnants of the moons at these spots the generation of the Ref PSF was done in an iteration loop. The iteration loop also corrected small distortions and flux differences between the images (called PSF matching, see Sect. 2.3) and further enhanced the astrometry of the images. The iteration loop is shown in Fig. 2. Its three main parts are: (1) generating a Ref PSF. The improved Ref PSF was generated from moon-cleaned individual images, calculated in the previous loop. (2) Improving the astrometry (RA and Dec) of Uranus and the moons. (3) Decomposing the individual images into matched PSFs at the position of Uranus and its five major moons. These are called the Uranus component and the Moon component (including all five moons) of a given image.

The iteration loop stopped when no significant change was found for the Ref PSF, nor any flux change for the moons.

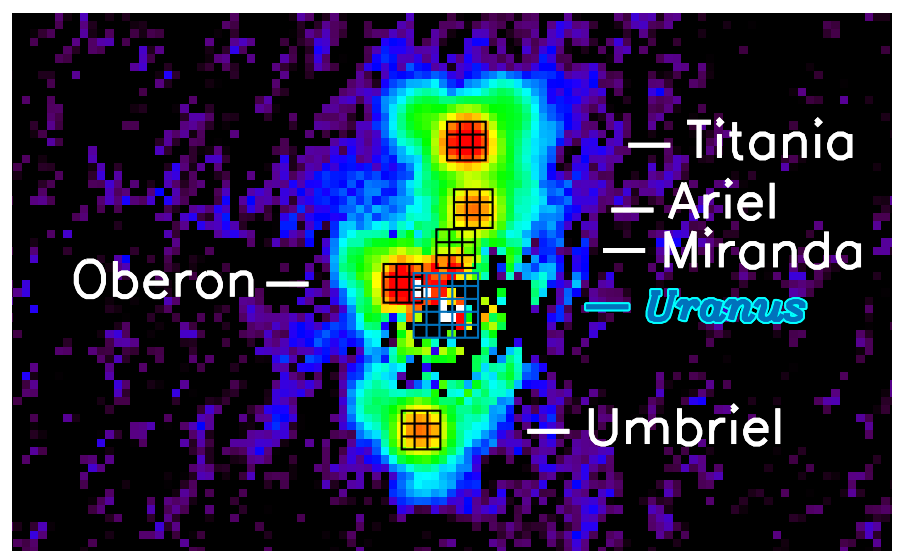

Fig. 3. Kernel positions of Uranus $(5 \times 5$, in blue $)$ and its moons $(3 \times 3$, in black) shown on the Uranus subtracted product of OBSID 1342211117+18. Overlapping kernel elements (Uranus and Oberon on this given example here) caused redundancy in the fit of these kernel elements. Rotational symmetries were introduced into the kernels to eliminate this issue. The flux scale of the image is the same as in Fig. 4.

\subsection{PSF matching}

Given the Uranus Ref PSF was generated from the measurements themselves, we obtained already good results using the simplest way to generate the Uranus component, namely, by using the Ref PSF from the previous iteration loop, multiplied by a simple relative flux parameter. This parameter was fitted for each measurement to take into account the flux changes of Uranus. Similarly, five flux parameters were used for the Moon component, fitted for each moon to take into account the relative flux difference of Uranus and its moons.

The Uranus PSF shape was changing slightly between images. To adjust these individual differences, we convolved the Ref PSF with normalised kernel matrices. Fitting $5 \times 5$ normalised kernel elements to the individual images improved the Uranus PSF subtraction near the centre of the PSF, making even the inner moons visible in some cases.

The PSF difference between the Uranus and its moons were clearly visible by leaving doughnut artefacts at the residual images of the moons. The use of a simple $3 \times 3$ sharpening kernel for the moon PSFs completely eliminated this issue, which had clearly originated from differences in their PSF size. The moons as well as Uranus had the same small distortions on the same image, therefore, we applied the sharpening kernel to the (already PSF-matched) Uranus component of a given image instead of the Ref PSF. The Moon component of an image was generated by shifting the Moon PSF to the moon positions at a given epoch and multiplied by the relative flux parameter of each moon.

The optimal sizes of the kernels change with wavelength. In order to have the same number of free parameters and constraints for all wavelengths, we implemented a spatial scale factor for the kernels. In this scaled kernel image convolution, the kernel values were used to weight the $-2 \mathrm{~d},-1 \mathrm{~d}, 0 \mathrm{~d}, 1 \mathrm{~d}, 2 \mathrm{~d}$ distance units shifted Ref PSF instances around Uranus in $X$ and $Y$-direction. Where the $\mathrm{d}$ units were $\mathrm{d}_{70}=1.5, \mathrm{~d}_{100}=1.25$ and $\mathrm{d}_{160}=1$ map pixels for the 70, 100, and $160 \mu \mathrm{m}$ images, respectively. Finally, all shifted elements were added together and multiplied by a relative flux parameter. We note here that this scaled kernel image convolution becomes a traditional image convolution with $\mathrm{d}=1$ pixel shift distance unit.

Figure 3 shows an example of the kernels. The $5 \times 5$ Uranus kernel is in blue and the $3 \times 3$ moon kernels are in black. An 

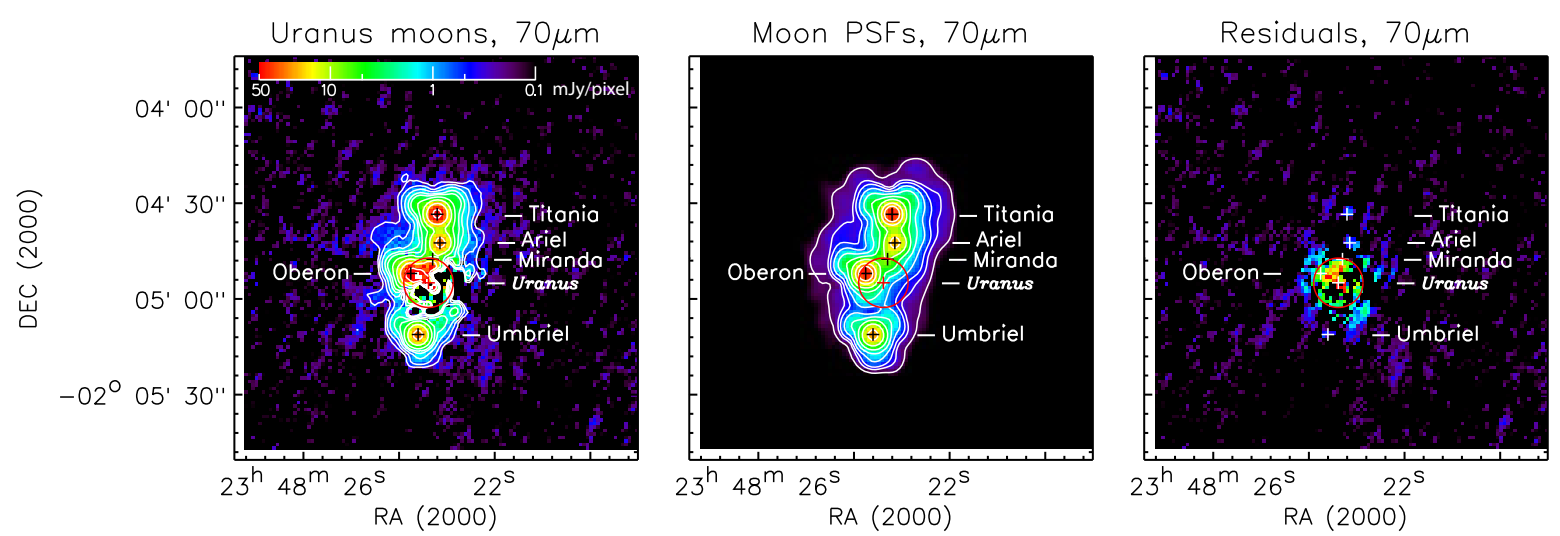

Fig. 4. $70 \mu \mathrm{m}$ PSF photometry of Uranian moons for OBSIDs 1342211117+18 on OD 579. Left: actual moon map after subtraction of the convolved Uranus PSF. The centres of the five moons are marked by black crosses and are labelled. The red cross inside the red circle indicates the approximate centre of the Uranus PSF. The circle has a radius of 7". 8 and circumscribes an area of significant PSF subtraction residuals (see right figure) inside which the photometric S/Ns are degraded (cf. Fig. 5). Middle: moon component of the image done by fitting a sharpening kernel and relative flux parameter for the reference PSF at the position of each moon (indicated by black crosses). This Moon component map recovers also intensity inside the circle area where the PSFs are disturbed in the map with the Uranus PSF subtracted. The moon PSF map is displayed with a larger dynamic range than the moon map. Right: residuals map (Moon map minus Moon PSF map) providing a judgement of the quality of the fit. The centres of the five moons and Uranus are marked here by white crosses and are labelled. For comparability, we used the same flux scale for all three images.

example of a fitted Moon component can be seen in the middle and residual image at the right in Fig. 4.

The major step in the iteration loop was to fit these kernels and flux parameters to each individual image. For the fitting parameters, the crucial point was to find a good balance between constraints and free parameters. The constraints were:

(1) until the very last iteration loop the flux of each moon was set constant for all observation epochs. This was crucial, because with this constraint the flux of a given moon was fitted dominantly to those epochs where it was farther away from the centre of Uranus due to the higher $\mathrm{S} / \mathrm{N}$ of the image at those pixels. The noise estimate was taken from the associated standard deviation map of the image product.

(2) Although the optimal kernels were not symmetric for all individual images, it was crucial to impose symmetry on the kernels. The PSFs of the nearby moons were overlapping with some image convolution elements, making the fit redundant for their kernel elements. For example, Fig. 3 displays where the kernels of Uranus and Oberon are overlapping. This redundancy would incorrectly elevate some of the kernel components of Oberon, reducing the Uranus kernel values proportionally. Implementing rotational symmetry for the kernels solved these redundancies. The Uranus kernel therefore was an average of two $5 \times 5$ kernels with $180^{\circ}$ and $120^{\circ}$ rotation symmetric elements.

(3) The more point-like Moon PSF was generated by a convolution of the Uranus component with the simplest (twoparameter) $90^{\circ}$ rotation-symmetric $3 \times 3$ normalised sharpening kernel. These fitted kernel elements were constant for all the epochs and the same for all moons as the relative diameter ratios of Uranus and its moons can be considered as constant.

(4) The last free parameters to be fitted were the $X$ and $Y$ spatial offsets of the images to improve the relative positions of the individual PSFs. The PSF subtraction is very sensitive to any offset. An uncertainty of $\approx 100$ mas for the Uranus centre would result in a quite significant residual pattern.

(5) In the last iteration loop, all previously fitted parameters were fixed, but the constant moon flux constraint was released. This last fit showed the variability of the moon fluxes from their averages for each epoch.

\subsection{PSF subtraction}

After fitting of all parameters to all individual images at the same time, two intermediate outputs were generated. First is the Uranus component-subtracted images. Second is the Moon component-subtracted images for Ref PSF generation at the beginning of the next iteration loop. This ensures that remnants of the moons on the Ref PSF are gradually removed with each iteration.

After the last iteration loop, the Uranus and Moon components were saved into the FITS files of the final moon map products. Subtracting both the Uranus and Moon components give the residual image. The residual image seen at the right of Fig. 4 clearly proves the correctness of the fit parameters and the correct balance of free fit parameters and constraints.

\section{Maps of the Uranian moons}

All data products with the PSF subtracted maps and including the convolved Uranus PSF and the moon PSFs in additional extensions will be available in FITS format as Herschel Highly Processed Data Products (HPDPs) ${ }^{3}$ in the Herschel Science Archive.

Figures A.1 to A.2 show the final actual maps of the Uranian moon constellations with the Uranus PSF subtracted for the five observation epochs. The corresponding scan and cross-scan maps have been averaged. It is clear that there is an inner area where the PSF subtraction does not work perfectly. This area is quantified by the results illustrated in Fig. 5 .

\section{Photometry of the Uranian moons}

The PSF photometry of the moons is a side product of our PSF subtraction itself, as we have to fit and subtract the moons to get a moon-cleared image for the Ref PSF generation. In comparison with aperture photometry, the constraint of knowing the exact PSF shape provides extra information to the PSF photometry, giving better results in crowded fields for overlapping sources.

\footnotetext{
https://www. cosmos. esa.int/web/herschel/

highly-processed-data-products
} 

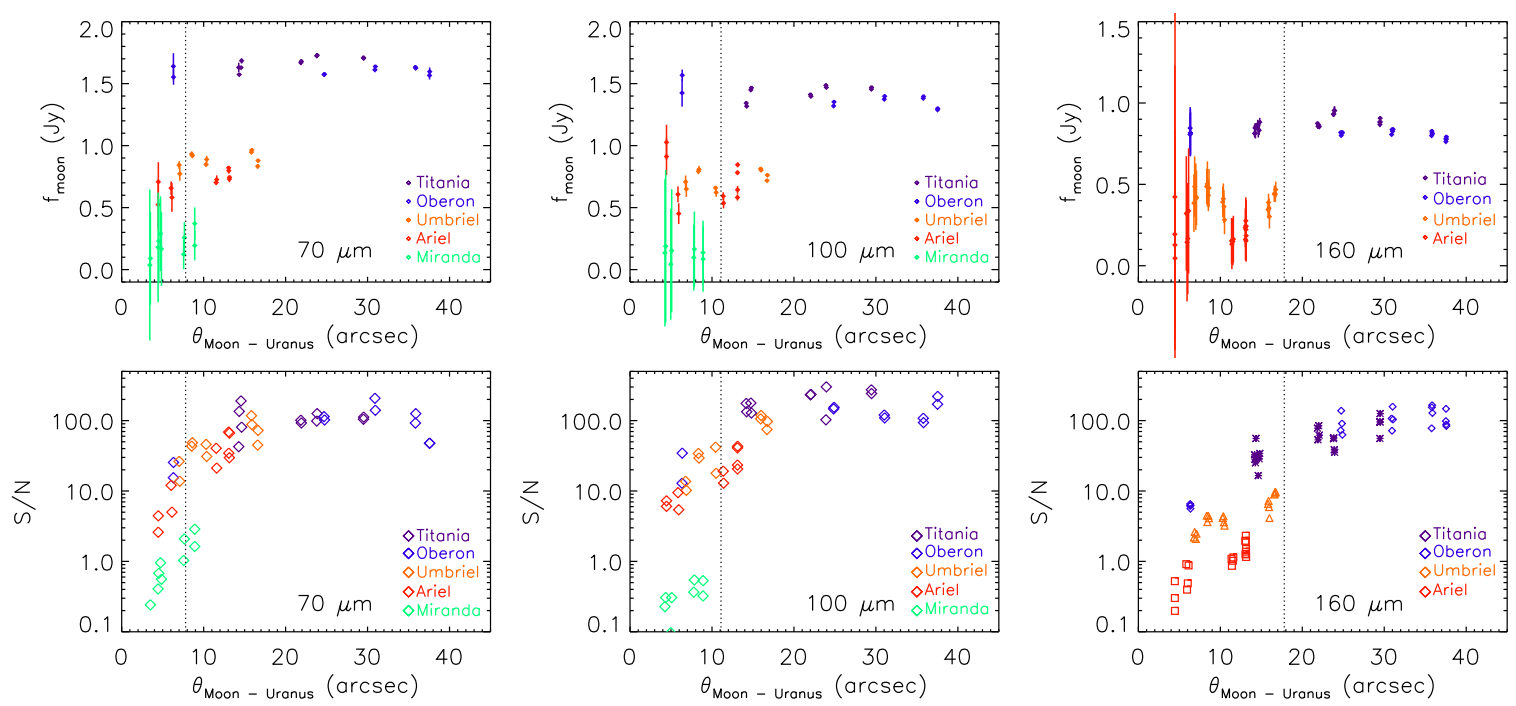

Fig. 5. Upper panel: derived fluxes $f_{\text {moon }}$ from PSF photometry and their uncertainties $\sigma_{\text {tot }}$ depending on the distance of the Uranian moon from the Uranus position for the 70,100 , and $160 \mu \mathrm{m}$ filter, respectively. Lower panel: corresponding signal-to-noise $\operatorname{ratios}\left(S / N=\frac{f_{\text {moon }}}{\sigma_{\text {tot }}}\right)$. The dashed vertical line at $\approx 7^{\prime \prime} 8,11^{\prime \prime} 1$, and $17^{\prime \prime} 8$, respectively (scaling with $\lambda_{\mathrm{c}}$ of the filter) indicates a radius inside which the uncertainty increases and the $\mathrm{S} / \mathrm{N}$ degrades noticeably due to PSF residuals.

To get additional confidence in our PSF photometry, we also performed standard aperture photometry whenever any moon was well-separated from Uranus.

\subsection{PSF photometry}

An example of PSF photometry fit results is shown in Fig. 4 for the combined scan and cross-scan map of OBSIDs $1342211117+18$, from which $70 \mu \mathrm{m}$ photometry for all five moons can be obtained. The PSF images of Oberon and Miranda are disturbed in the residual map due to imperfect Uranus PSF subtraction in this central area, nevertheless a significant fraction of the moon PSF is available to recover the total flux and reconstruct the intensity distribution. As already mentioned earlier in this paper, the fitting algorithm weights the pixels with their sigma value using the associated standard deviation map of the image product. In the case of Miranda, the PSF is fitted dominantly to this outer part of the Uranus PSF, where the S/N of the pixels is higher than the ones closer to the Uranus centre. Of course the uncertainty of the PSF fit worsens if only part of the PSF is available.

The unitless PSF flux fit parameters were relative fluxes used to weight the Ref PSF. To get the flux in Jy from these weights, they have to be multiplied with the aperture photometry of the Ref PSF, in other words, the average flux of Uranus over the measurements. The flux uncertainties were calculated the same way from the unitless 1-sigma parameter error values of the PSF fit parameters. This is the second component in our photometric uncertainty assessment. The related value is listed under $\sigma_{\mathrm{par}}$.

\subsection{Aperture photometry}

Based on the Uranus subtracted products, we also performed standard aperture photometry, as described in the PACS Handbook (Exter et al. 2018), Sect. 7.5.2. Subtracting all other moons from the product (except the one we were measuring) clearly enhanced the aperture photometry results. Still, it was possible when a given moon was well separated from Uranus at a given epoch. This is mainly the case for Oberon and Titania, while
Table 2. Comparison of PSF photometry with standard aperture photometry for a number of measurements, $n$, when an Uranian moon was far enough off Uranus and the other moons to allow relatively undisturbed aperture measurements.

\begin{tabular}{rcc}
\hline \hline Filter & $n$ & $\frac{f^{\mathrm{PSF}}}{f^{\text {aper }}}$ \\
\hline 70 & 26 & $1.030 \pm 0.003$ \\
100 & 22 & $1.032 \pm 0.005$ \\
160 & 28 & $1.036 \pm 0.004$ \\
\hline
\end{tabular}

unfortunately the number of comparison cases for Umbriel and Ariel is quite limited, in particular at $160 \mu \mathrm{m}(70 \mu \mathrm{m}: 8$ cases, $100 \mu \mathrm{m}$ : 4 cases, $160 \mu \mathrm{m}$ : 0 cases).

The detailed comparison of PSF photometry with aperture photometry has been compiled in Table A.7. A statistical overview is given in Table 2. From this, it can be seen that the consistency of the two photometric methods is very good (within 3-4\%), thus confirming the principal quality of our PSF photometry procedure. This does not, however, exclude that individual fits may be unreliable or even fail, particularly in areas with high PSF residuals or confusion from close sources. The uncertainty of the fit gives then already good advice on the reliability.

The aperture photometry shows on average a systematic $3-4 \%$ negative flux offset with regard to the PSF photometry. This flux loss was a result of the small apertures and sky radii to achieve good residual rejection.

\subsection{Photometry results}

In Fig. 5, we plotted the PSF photometry fluxes and their uncertainties and the corresponding $\mathrm{S} / \mathrm{N}$ of the individual measurements depending on the distance of the Uranian moon from the Uranus position for each filter. As a general feature, we note that the uncertainties increase and, hence, the $\mathrm{S} / \mathrm{N}$ degrades noticeably inside a certain radius, which is $\approx 77^{\prime \prime} 8,11^{\prime \prime}$. 1 , and $177^{\prime \prime} 8$ for 70,100 , and $160 \mu \mathrm{m}$, respectively (these radii scale with $\lambda_{\mathrm{c}}$ of the filter). This is due to PSF residuals as seen in Figs. A.1-A.3. 
Table 3. Mean fluxes of the Uranian moons (Eq. (3)) calculated from a weighted mean moon-to-Uranus flux ratio (Eq. (1)) and a mean Uranus flux (Eq. (2)) over the Herschel observation campaign.

\begin{tabular}{|c|c|c|c|c|c|c|c|c|c|}
\hline Object & $n_{70}$ & $\begin{array}{c}\left(\frac{f_{\text {moon }}}{f_{\text {Uranus }}}\right)_{70}^{\text {mean }} \\
\left(10^{-3}\right)\end{array}$ & $\begin{array}{c}f_{\text {object }, 70}^{\text {mean }} \\
\text { (Jy) }\end{array}$ & $n_{100}$ & $\begin{array}{c}\left(\frac{f_{\text {moon }}}{f_{\text {Uranus }}}\right)_{100}^{\text {mean }} \\
\left(10^{-3}\right)\end{array}$ & $\begin{array}{c}f_{\text {object, } 100}^{\text {meen }} \\
(\mathrm{Jy})\end{array}$ & $n_{160}$ & $\begin{array}{c}\left(\frac{f_{\text {moon }}}{f_{\text {Uranus }}}\right)_{160}^{\text {mean }} \\
\left(10^{-3}\right)\end{array}$ & $\begin{array}{c}f_{\text {object, } 160}^{\text {meen }} \\
(\mathrm{Jy})\end{array}$ \\
\hline $\mathrm{T}$ & 10 & $1.931 \pm$ & 2 & 10 & $1.619 \pm$ & 123 & 20 & $1.317 \pm$ & $0.873=$ \\
\hline Oberon & 10 & $1.847 \pm 0.0067$ & .006 & 10 & $1.537 \pm 0.0074$ & $1.351=$ & 20 & $1.217 \pm 0.0$ & 0.003 \\
\hline Umbriel & 10 & $1.055 \pm 0.0140$ & 12 & 10 & $0.869 \pm$ & 0.764 & 20 & $0.613 \pm 0.0187$ & $0.406=$ \\
\hline Ariel & 10 & $0.876 \pm 0.0150$ & $0.754 \pm 0.013$ & 10 & $0.799 \pm 0.0428$ & $0.702 \pm 0.038$ & 20 & $0.294 \pm 0.0171$ & $0.195 \pm 0.011$ \\
\hline Miranda & 10 & $0.261 \pm 0.0349$ & $0.225 \pm 0.030$ & 8 & $0.135 \pm 0.0164$ & $0.119 \pm 0.016$ & - & - & - \\
\hline Uranus & 10 & & $861.287 \pm 0.535$ & 10 & & $879.061 \pm 0.488$ & 20 & & $663.011 \pm 0.4$ \\
\hline
\end{tabular}

Notes. $\sigma_{\text {tot }}$ of the individual moon photometry was used as weight. The applied mean distance (20.024 AU) normalised Uranus flux is given in the last line. $n_{70}, n_{100}$ and $n_{160}$ give the number of reliable measurements used in the determination of $\frac{f_{\text {moon }}}{f_{\text {Uranus }}}$.

It should be noted that negative fluxes and, hence, the negative S/Ns do not occur since the PSF fit produces either positive fluxes or fails. For the photometry of the individual moons, the following can be concluded:

- the S/Ns of all Titania measurements are $>10$, so that all measurements should be very reliable;

- the S/Ns of the Oberon measurements for epochs 2-5 are all $>10$, so that all these measurements should be very reliable. Regarding the measurements of the first epoch, the moon is inside the critical radius. Nevertheless $\mathrm{S} / \mathrm{N}$ at 70 and $100 \mu \mathrm{m}$ are still $\gtrsim 10$, so that their quality should be medium. At $160 \mu \mathrm{m}$ the $\mathrm{S} / \mathrm{Ns}$ are $<10$, so that this photometry is less reliable;

- for Umbriel, the S/Ns of the 70 and $100 \mu \mathrm{m}$ measurements of epochs 1 and 5, which are outside the critical radius, are of very high quality. The corresponding $160 \mu \mathrm{m}$ fluxes have $S / N \mathrm{~s}$ $\lesssim 10$, so that they are less reliable. The S/Ns for the 70 and $100 \mu \mathrm{m}$ measurements of epochs $2-4$ are between $10-50$, so that their quality should be still medium. However, the corresponding $160 \mu$ m fluxes have $\mathrm{S} / \mathrm{Ns}$ between $2-5$, so that this photometry is less reliable;

- for Ariel, the S/Ns of the 70 and $100 \mu \mathrm{m}$ measurements of epochs 1 to 3 have medium to high quality $(\gtrsim 10-<100)$. The S/Ns of the 70 and $100 \mu \mathrm{m}$ measurements of epochs 4 and 5 are $\lesssim 10$, so that they are less reliable. The S/Ns of all $160 \mu \mathrm{m}$ measurements are $\lesssim 3$, so that they are likely to be quite inaccurate;

- for Miranda, which is considerably fainter than the other four moons and always close to Uranus, the S/Ns of the $70 \mu \mathrm{m}$ measurements of epochs 1 and 3 are in the range between 1 and 3. These measurements indicate the order of flux, but they are not very reliable. All other measurements at 70 and $100 \mu \mathrm{m}$ have $S / N$ s $\lesssim 1$, so that individual measurements are not reliable at all. At $160 \mu \mathrm{m} S / N \mathrm{~s}$ are $\ll 1$.

Small S/Ns indicate that there are some restriction in the subsequent analysis. It is important to bear in mind that this is not a deficiency of the observational design since the original design with just one modular mini scan map was meant to observe Uranus, so that the $\mathrm{S} / \mathrm{N}$ for the moons is, naturally, not optimal.

The results of photometry from the individual scan maps are given in Tables A.2-A.6. For completeness we compile the Uranus photometry in Table A.1. This table gives both the actually measured flux $f_{i, \text { mranus }}$ and a flux normalised to a reference distance $f_{\mathrm{i}, \text { Uranus }}^{\text {distancecrected }}$ which is needed in generating the PSF reference. The determination of the distance corrected Uranus flux is described below.
Table 3 provides an overview of the Uranian moon photometry with mean fluxes. For it a weighted mean moon-to-Uranus flux ratio was calculated from the individual photometry results listed in Tables A.1-A.6,

$\left(\frac{f_{\text {moon }}}{f_{\text {Uranus }}}\right)_{\lambda}^{\text {mean }}=\frac{\sum_{i=1}^{n}\left(\frac{f_{\mathrm{i} \text {,moon }}^{\text {measured }}}{f_{\mathrm{i}, \text { Uranusu }}^{\text {meanu }}}\right)_{\lambda}\left(\frac{1}{\sigma_{\mathrm{i}, \text { tot }}}\right)^{2}}{\sum_{i=1}^{n}\left(\frac{1}{\sigma_{\mathrm{i}, \text { tot }}}\right)^{2}}$,

using the $\sigma_{\mathrm{i}, \text { tot }}$ of the moon photometry as weights. For the calculation of the mean moon fluxes, a weighted mean Uranus flux at a mean distance of all Herschel observations is used. The mean Uranus distance is derived from the $\Delta_{\text {obs,i }}$ of the 20 individual observations $\left(\Delta_{\mathrm{obs} \text {,mean }}=\frac{\sum_{i=1}^{20} \Delta_{\mathrm{obs}, \mathrm{i}}}{20}=20.024 \mathrm{AU}\right.$; for the $\Delta_{\text {obs,i }}$ cf. Table A.1). Individual distance corrected Uranus fluxes $f_{\mathrm{i}, \text { Uranus }}^{\text {distancecorrected }}$ are determined by scaling the measured Uranus flux $f_{\mathrm{i}, \text { Uranus }}^{\text {measured }}$ with the correction factor $c_{\text {dist }}=\left(\frac{\Delta_{\mathrm{obs}, \mathrm{i}}}{\Delta_{\mathrm{obs} \text {,mean }}}\right)^{2}$ (see also Table B.1 for values of $c_{\text {dist }}$ per observation epoch). The weighted mean Uranus flux is then calculated as

$f_{\text {Uranus }, \lambda}^{\text {mean }}=\frac{\sum_{i=1}^{n} \frac{f_{\mathrm{i}, \text { Uranus }}^{\text {fistancecrected }}}{\sigma_{\mathrm{i}, \mathrm{tot}}^{2}}}{\sum_{i=1}^{n} \frac{1}{\sigma_{\mathrm{i}, \text { tot }}}}$

using the $\sigma_{i, \text { tot }}$ of the individual Uranus measurements as weights. These mean distance corrected fluxes of Uranus are listed in Table 3, too. The mean moon flux is then calculated as

$f_{\text {moon }, \lambda}^{\text {mean }}=\left(\frac{f_{\text {moon }}}{f_{\text {Uranus }}}\right)_{\lambda}^{\text {mean }} \times f_{\text {Uranus }, \lambda}^{\text {mean }}$.

The combined flux contribution of the four largest moons relative to the Uranus flux is $5.7 \times 10^{-3}, 4.8 \times 10^{-3}$, and $3.4 \times 10^{-3}$ at 70,100 , and $160 \mu \mathrm{m}$, respectively. Hence, earlier published photometry of Uranus (Müller et al. 2016) not subtracting the moon contribution is not invalidated by our new results. Rather, we specify more precisely the systematic error of the Uranus flux by its moons when using Uranus as a far-infrared prime flux calibrator. The fluxes in column $f_{\text {total }}$ of Table A.1 are very consistent with those in Table B.1, column "Flux; in Müller et al. (2016) $\left(\frac{f_{\text {tot }}^{\text {thispaper }}}{f_{\text {Urueller2016 }}^{\text {Muras }}}=1.005 \pm 0.005,1.009 \pm 0.003\right.$, and $1.014 \pm 0.004$ at 70 , 100 , and $160 \mu \mathrm{m}$, respectively). 

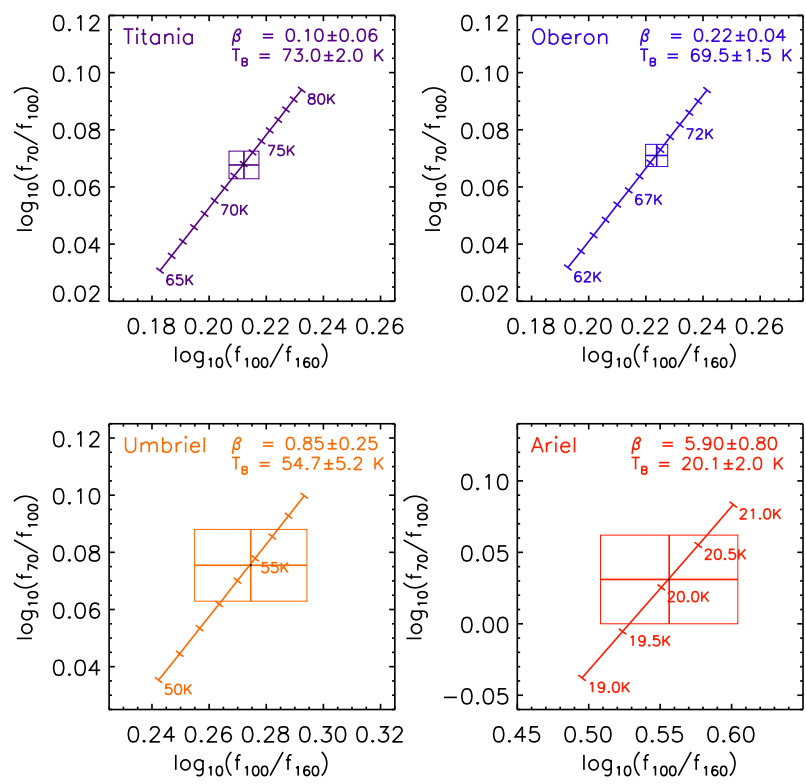

Fig. 6. PACS two-colour diagrams for the Uranian moons. The PACS fluxes are not colour corrected and refer to the PACS standard photometric reference SED $v \times f_{v}=$ const. The boxes with the central cross indicate the uncertainty range of the measured colours (determined by $\log _{10}\left(\frac{f_{\lambda_{1}}+\sigma_{\lambda_{1}}}{f_{\lambda_{2}}-\sigma_{\lambda_{2}}}\right)$ and $\log _{10}\left(\frac{f_{\lambda_{1}}-\sigma_{\lambda_{1}}}{f_{\lambda_{2}}+\sigma_{\lambda_{2}}}\right)$, respectively.) The straight lines with the temperature tick marks represent the colours of modified blackbodies calculated according to Eq. (4) for the displayed temperature range and with $\beta$ as indicated in the upper right corner of each panel. Derived $\beta$ and $\mathrm{T}_{\mathrm{b}}$ are used for a plausibility check of the PACS photometry.

No dependence of the moon fluxes on the distance to Uranus is expected since all the moons have orbits with small eccentricity. The variation of the angular separation of the moons to Uranus stands as a pure projection effect that is due to the inclination of the Uranian system.

Another plausibility check of the PACS photometry can be obtained from FIR two-colour diagrams. In Fig. 6, we show the individual two-colour diagrams for the Uranian moons. The PACS fluxes are not colour corrected and refer to the PACS standard photometric reference SED $v \times f_{v}=$ const. Modified blackbody functions $\frac{v^{\beta}}{v_{0}^{\beta}} \mathrm{B}_{v}\left(\mathrm{~T}_{\mathrm{b}}\right)$ are good first order approximations for dust emission. Emission from the surface regolith of satellites is usually well approximated by pure blackbody emission, that is, $\beta$ should be zero or small. We calculated the two PACS colours of modified blackbody emission as:

$\log _{10}\left(\frac{\lambda_{1}^{(2-\beta)} \times B_{\lambda}\left(\lambda_{1}, T\right) \times c c_{\lambda_{1}}}{\lambda_{2}^{(2-\beta)} \times B_{\lambda}\left(\lambda_{2}, T\right) \times c c_{\lambda_{2}}}\right)$.

The modified blackbody fluxes have been colour corrected $\left(\mathrm{cc}_{\lambda}\right)$ to the PACS photometric reference SED for a homogeneous comparison with the moon colours (cf. PACS Handbook, Exter et al. 2018, formula 7.20 for the calculation for any SED shape). We checked which combination of $\beta$ and $\mathrm{T}_{\mathrm{b}}$ best match the measured colours. Figure 6 shows the line for the best matching $\beta$ value and a range of $T_{\mathrm{b}}$ which crosses the measured combination of colours. For Titania and Oberon, the approximation of the measured colours by pure blackbodies is quite good since the match yields $\beta=0.10 \pm 0.06, T_{\mathrm{b}}=73.0 \mathrm{~K} \pm 2.0 \mathrm{~K}$ and $\beta=$ $0.22 \pm 0.04, T_{\mathrm{b}}=69.5 \mathrm{~K} \pm 1.5 \mathrm{~K}$, respectively. For Umbriel, we find $\beta=0.85 \pm 0.25, T_{\mathrm{b}}=54.7 \mathrm{~K} \pm 5.2 \mathrm{~K}$, which shows that the $160 \mu \mathrm{m}$ flux is somewhat too low, so that the $\log \left(\frac{f_{100}}{f_{160}}\right)$ value is too high, thus requiring higher $\beta$ values. For Ariel, the fit gives $\beta=$ $5.9 \pm 0.8, T_{\mathrm{b}}=20.1 \mathrm{~K} \pm 2.0 \mathrm{~K}$, which is a completely unphysical spectral energy distribution solution for this moon. We conclude that the mean $160 \mu \mathrm{m}$ flux is far too low (about a factor of 2) and unreliable, as suggested by the $\mathrm{S} / \mathrm{N}$ analysis above. On the other hand, the mean 70 and $100 \mu \mathrm{m}$ photometry appears to be adequate for all four moons, since the $\log \left(\frac{f_{70}}{f_{100}}\right)$ values are all similar. Because of the partial deficiency or incompleteness of the measured SEDs, we derived colour correction factors for the PACS photometry from the best fitting models (see Table 7).

\section{Auxiliary thermal data}

In addition to the new PACS measurements, we searched in the literature to find more thermal data for the Uranian satellites. Brown et al. (1982) presented standard broad-band $Q$ filter measurements taken by the $3-\mathrm{m} \mathrm{IRTF}^{4}$ telescope. We re-calibrated the $Q$-band magnitudes (after applying the listed monochromatic correction factors and taking the specified -3.32 mag for $\alpha$ Boo) with the template flux of $185.611 \mathrm{Jy}$ at $20.0 \mu \mathrm{m}$ (Cohen et al. 1996). The resulting flux densities are given in Table 4.

An important set of measurements was taken by SpitzerIRS $(14-37 \mu \mathrm{m})$ (Houck et al. 2004). We took the reduced and calibrated low-resolution spectra (Lebouteiller et al. 2011) and high-resolution spectra (Lebouteiller et al. 2015) from CASSIS $^{5}$, all related to the Spitzer Program ID $71^{6}$. The programme includes thermal emission spectroscopy between 10 and $40 \mu \mathrm{m}$ for Uranus' synchronous satellites (among other objects), observing the leading and trailing hemispheres at large separations from the planet. These observations were taken under aspect angles between $96.8^{\circ}$ and $104.6^{\circ}$, that is, close to an equator-on view of Uranus and its four satellites. An overview of these observations is given in Table 5.

The "optimal" extraction of the spectra from the CASSIS data assumes a perfect point-like source which is certainly the case for the Uranian satellites at 18-20 AU distance from the $0.85 \mathrm{~m}$ Spitzer Space Telescope. We also looked into the highresolution scans, but they only cover the longer wavelength range $(19.5-36.9 \mu \mathrm{m})$ and from the comparison with the low-resolution spectra we concluded that they do not add any new information. At longer wavelengths $(>22 \mu \mathrm{m}$ for Umbriel and $>30 \mu \mathrm{m}$ for Oberon), the CASSIS spectra (both, low- and high-resolution ones) show significant additional fluxes, which probably originate from the Uranus PSF (cf. Figs. 9 and 7, respectively). The Ariel spectra have fluxes which are at least a factor of 2-3 too high and it seems the data are still affected by the influence of Uranus (cf. Fig. 10). We eliminated those parts which show a strong deviation from a typical satellite thermal emission spectrum. We rebinned the spectra down to 10-15 wavelength points and added $10 \%$ to the measurement errors to account for absolute flux calibration uncertainties in the close proximity of a very bright source.

Cartwright et al. (2015) presented IRTF/SpeX ( 0.81$2.42 \mu \mathrm{m})$ and Spitzer/IRAC $(3.6,4.5,5.8$, and $8.0 \mu \mathrm{m})$ measurements. But even at $8 \mu \mathrm{m}$, the measured fluxes are dominated by reflected sunlight. In the most favourable case, the thermal contribution was still well below $10 \%$. We therefore excluded these measurements from our radiometric studies.

Hanel et al. (1986) studied the Uranian system with infrared observations obtained by the infrared interferometer

\footnotetext{
4 Infrared Telescope Facility on Mauna Kea, Hawaii.

5 https://cassis.sirtf.com/atlas/welcome.shtml

6 ID 71: Observations of Outer Solar System Satellites and Planets; PI: J. R. Houck.
} 
Table 4. Flux densities and uncertainties at $20.0 \mu \mathrm{m}$ based on measured $Q$-band magnitudes from Brown et al. (1982) and re-calibrated via the reference standard star $\alpha$ Boo.

\begin{tabular}{lcccccc}
\hline \hline Object & MJD & $\begin{array}{c}r_{\text {helio }} \\
(\mathrm{AU})\end{array}$ & $\begin{array}{c}\Delta_{\text {obs }} \\
(\mathrm{AU})\end{array}$ & $\begin{array}{c}\alpha \\
(\mathrm{deg})\end{array}$ & $\begin{array}{c}\text { ang-sep } \\
\left({ }^{\prime \prime}\right)\end{array}$ & $\begin{array}{c}f_{20} \\
(\mathrm{Jy})\end{array}$ \\
\hline Ariel (UI) [701] & 45111.50 & 18.879 & 17.867 & 0.09 & 14.65 & $0.142 \pm 0.026$ \\
Umbriel (UII) [702] & 45109.33 & 18.879 & 17.869 & 0.21 & 19.86 & $0.131 \pm 0.018$ \\
Titania (UIII) [703] & 45109.50 & 18.879 & 17.868 & 0.20 & 33.31 & $0.250 \pm 0.024$ \\
Oberon (UIV) [704] & 45108.40 & 18.880 & 17.871 & 0.26 & 43.23 & $0.280 \pm 0.038$ \\
\hline
\end{tabular}

Notes. Data were taken in May 1982 with the IRTF (Miranda was not part of the study). $r_{\text {helio }}$ is the light-time corrected heliocentric range, $\Delta_{\text {obs }}$ is the range of target centre wrt. the observer, i.e. IRTF, $\alpha$ is the phase angle and "ang-sep" is the apparent angular separation from Uranus. The aspect angle during the measurements was around $163.5^{\circ}$ which means that IRTF saw mainly the South-pole region of Uranus and the four satellites.

Table 5. Overview of the Spitzer-IRS CASSIS spectra.

\begin{tabular}{|c|c|c|c|c|c|c|c|c|}
\hline Object & MJD & $\begin{array}{l}r_{\text {helio }} \\
\text { (AU) }\end{array}$ & $\begin{array}{c}\Delta_{\text {obs }} \\
(\mathrm{AU})\end{array}$ & $\begin{array}{c}\alpha \\
(\operatorname{deg})\end{array}$ & $\begin{array}{c}\text { ang-sep } \\
\left({ }^{\prime \prime}\right)\end{array}$ & AORkey & hemisp. & $\begin{array}{l}\text { UsefulSpectrum } \\
(\mu \mathrm{m})\end{array}$ \\
\hline \multirow[t]{2}{*}{ Ariel (UI) [701] } & 53563.98055 & 20.067 & 19.547 & 2.54 & 13.45 & 4521984 & $\mathrm{~L}$ & - \\
\hline & 53323.25416 & 20.056 & 19.724 & 2.76 & 13.33 & 4522240 & $\mathrm{~T}$ & - \\
\hline \multirow[t]{2}{*}{ Umbriel (UII) [702] } & 53695.76250 & 20.073 & 19.673 & 2.69 & 18.63 & 4522496 & $\mathrm{~L}$ & $14.1-22.0$ \\
\hline & 53183.96250 & 20.049 & 19.582 & 2.62 & 18.72 & 4522752 & $\mathrm{~T}$ & $14.1-22.0$ \\
\hline \multirow[t]{2}{*}{ Titania (UIII) [703] } & 53181.21458 & 20.048 & 19.624 & 2.67 & 30.61 & 4523008 & $\mathrm{~L}$ & $14.1-37.3$ \\
\hline & 53716.59166 & 20.074 & 20.017 & 2.89 & 30.08 & 4523264 & $\mathrm{~T}$ & $14.1-37.3$ \\
\hline \multirow[t]{2}{*}{ Oberon (UIV) [704] } & 53184.28680 & 20.049 & 19.577 & 2.61 & 41.09 & 4523520 & $\mathrm{~L}$ & $14.1-30.0$ \\
\hline & 53325.65486 & 20.056 & 19.763 & 2.80 & 40.70 & 4523776 & $\mathrm{~T}$ & $14.1-30.0$ \\
\hline
\end{tabular}

Notes. $r_{\text {helio }}$ is the light-time corrected heliocentric range, $\Delta_{\text {obs }}$ is the range of target centre wrt. the observer, i.e. Spitzer, $\alpha$ is the phase angle and "ang-sep" is the apparent angular separation from Uranus. The observations were designed to observe either the leading or the trailing hemisphere, which is indicated in column "hemisp". "UsefulSpectrum" indicates the wavelength range not affected by Uranus stray-light; in the case of Ariel the whole spectrum is affected.

Table 6. TPM input parameters for the radiometric calculations and the interpretation of the obtained/available mid-/far-IR flux densities.

\begin{tabular}{lcccccc}
\hline \hline Object & $\begin{array}{c}H_{\mathrm{V}} \\
(\mathrm{mag})\end{array}$ & $A$ & $q$ & $p_{\mathrm{V}}$ & $\begin{array}{c}D_{\text {eff }} \\
(\mathrm{km})\end{array}$ & $\begin{array}{c}\text { Orbital period } \\
(\text { days })\end{array}$ \\
\hline Ariel (UI) [701] & 0.99 & $0.230 \pm 0.025$ & $0.43 \pm 0.05$ & 0.53 & 1159.0 & 2.520 \\
Umbriel (UII) [702] & 1.76 & $0.100 \pm 0.010$ & $0.39 \pm 0.04$ & 0.26 & 1170.0 & 4.144 \\
Titania (UIII) [703] & 0.78 & $0.170 \pm 0.015$ & $0.46 \pm 0.05$ & 0.37 & 1578.0 & 8.706 \\
Oberon (UIV) [704] & 0.99 & $0.140 \pm 0.015$ & $0.44 \pm 0.05$ & 0.31 & 1522.0 & 13.463 \\
Miranda (UV) [705] & 3.08 & $0.200 \pm 0.030$ & $0.44 \pm 0.07$ & 0.45 & 474.0 & 1.413 \\
\hline
\end{tabular}

Notes. The numbers are taken from Karkoschka (2001): $H_{\mathrm{V}}$ are the mean values between $V_{\max }$ and $V_{\min }$ with an uncertainty of 0.04 mag, $D_{\text {eff }}$ was calculated from the specified radii (both from Table IV). The phase integral $q$ and the Bond albedo $A$ are from Table VII $\left(q\right.$ and $q I_{0} / F$, respectively). The geometric albedo $p_{V}$ was calculated via $A=p q$, with $p / p_{V} \approx 1.0$ (Morrison \& Lebofsky 1979).

spectrometer (IRIS) on Voyager 2. The measurements were taken for Miranda and Ariel and cover the range between 200 and $500 \mathrm{~cm}^{-1}(20-50 \mu \mathrm{m})$. The South polar region was seen for both targets (under phase angles of $38^{\circ}$ for Miranda and $31^{\circ}$ for Ariel). They measured a maximum brightness temperature near the subsolar point, $T_{\mathrm{SS}}$, of $86 \pm 1 \mathrm{~K}$ and $84 \pm 1 \mathrm{~K}$ for Miranda and Ariel, respectively. We tested our final model solutions against these two brightness temperatures.

\section{Thermophysical modelling of the Uranian moons}

For the interpretation of the available thermal IR fluxes, we used the thermophysical model (TPM) by Lagerros (1996, 1997, 1998) and Müller \& Lagerros (1998, 2002). The calculations are based on the true observer-centric illumination and observing geometry for each data point (topocentric for IRTF, HerschelSpitzer-centric). The model considers a one-dimensional heat conduction into the surface, controlled by the thermal inertia. The surface roughness is implemented via segmented hemispherical craters where the effective rms of the surface slopes is controlled by the crater depth-to-radius ratio and the surface coverage of the craters (Lagerros 1998). Additional input parameters are the object's thermal mid-/far-IR emissivity (assumed to be 0.9 ), the absolute $V$-band magnitudes $H_{\mathrm{V}}$ of the Uranian satellites, the phase integrals $q$, the measured sizes $D_{\text {eff }}$ and albedos $\mathrm{p}_{V}$. The $H_{\mathrm{V}}$ is only relevant in cases where we solve for radiometric size-albedo solutions. In cases where we keep the size fixed, $H_{\mathrm{V}}$ is not used. Table 6 summarises these values. For the satellites' rotation properties, we assume a spin-axis orientation perpendicular to Uranus' equator (orbital inclinations are below 
$0.5^{\circ}$, only for Miranda it is $4.2^{\circ}$ ), and a (presumed) synchronous rotation.

Using the above properties (and their uncertainties) allows us now to determine the moons' thermal properties. We vary the surface roughness from very smooth (rms of surface slopes $<0.1$ ) up to very rough surfaces (rms of surface slopes $>0.7$ ). In addition, low-conductivity surfaces can have very small thermal inertias (here, we use a lower limit of $0.1 \mathrm{~J} \mathrm{~m}^{-2} \mathrm{~s}^{-0.5} \mathrm{~K}^{-1}$ ) and compact solid surfaces have high conductivities (we consider thermal inertias up to $100 \mathrm{~J} \mathrm{~m}^{-2} \mathrm{~s}^{-0.5} \mathrm{~K}^{-1}$ ).

One problem of radiometric studies in general is related to objects seen pole-on, or very close to pole-on (especially for distant objects where the Sun and observer face the same part of the surface). In these cases, there is no significant heat transfer to the night side and it is much more difficult to constrain the object's thermal properties. A pole-on geometry is connected to an aspect angle of $0^{\circ}$ (north pole) or $180^{\circ}$ (south pole), while an equator-on geometry has $90^{\circ}$. During the 1980 s (including the IRTF measurements, but also the time of the Voyager 2 flyby) mainly the south pole region (of Uranus and also the synchronous satellites) was visible, the 2004/2005 Spitzer measurements were taken at aspect angles between about $97^{\circ}$ and $105^{\circ}$, the 2010-2012 Herschel observations saw the Uranus system under aspect angles between about $70^{\circ}$ and $81^{\circ}$, meaning that both were close to an equator-on view. The phase angles are typically small (below $3^{\circ}$ ) and the measured signals are in all cases related to almost fully illuminated objects.

Representative examples of our thermophysical models for the epoch 2011-07-12 (period 2) covering the wavelength range between 5-300 $\mu \mathrm{m}$ are shown for Oberon, Titania, Umbriel, and Ariel in Figs. 7-10, including the corresponding surface temperature maps. All derived (and approved) flux densities for the 5 satellites, as well as the corresponding best TPM SEDs will be made available by the Herschel Science Centre through 'User Provided Data Products' in the Herschel Science Archive ${ }^{7}$. Our Herschel flux densities and the auxiliary photometry shall also be imported into the 'Small Bodies: Near and Far' (SBNAF) data base $^{8}$ for thermal infrared observations of Solar System's small bodies (Szakáts et al. 2020).

At the time of Voyager flyby, when the south pole of the moons was facing the Sun, maximum surface temperatures reached or exceeded $85 \mathrm{~K}$, but nighttime polar temperatures are predicted to drop to 20 or $30 \mathrm{~K}$, because each pole spends about $40 \mathrm{yr}$ in darkness (Veverka et al. 1991). This means that under an illumination geometry close to pole-on the satellite surface is hotter than under an illumination geometry close to equator-on when heat transport to the night side results in a colder surface temperature. Therefore, a simple scaling of photometric measurements taken under different illumination geometry by just correcting for different ranges of target centre with regard to the observer will not allow a direct comparison. This has to be kept in mind for Figs. 7-10, where there seems to be some flux inconsistency between the IRTF fluxes and the thermophysical model fluxes matching the PACS observations. When taking the illumination geometry at the time of the IRTF measurements into account for the TPM, the consistency is very good, that is, for Titania $\frac{f_{\mathrm{IRTF}}}{f_{\mathrm{TPM}(\mathrm{t}(\mathrm{RTF})}}=\frac{0.250 \mathrm{Jy}}{0.233 \mathrm{Jy}}$.

As part of the analysis we also looked into differences between the leading (LH) and trailing hemispheres (TH) of the satellites. The tidally-locked and large satellites display stronger $\mathrm{H}_{2} \mathrm{O}$ ice bands on the leading hemispheres, but this

\footnotetext{
http://archives.esac. esa.int/hsa/whsa/

8 https://ird.konkoly.hu/
}

effect decreases with distance from Uranus. In addition, Titania and Oberon show spectrally red material on their leading hemisphere. Cartwright et al. (2018) discuss the possible origin of the hemispherical differences and speculate that inwardmigrating dust from the irregular satellites might be the cause of the observed $\mathrm{H}_{2} \mathrm{O}$ ice bands and red material differences in the two hemispheres. Since the IRTF measurements viewed only the south pole regions, the measured fluxes did not allow such a separation. The Spitzer-IRS measurements were aiming for epochs were either the leading or trailing faces were seen. The measurements were timed for maximum elongation from Uranus, which are close to the epochs of the minimum and maximum heliocentric range-rate values. This was possible since the Uranus system was seen almost equator-on. The Herschel measurements were not timed to catch the objects at their rangerate maxima. Therefore, we consider Herschel observations as leading and trailing cases, if the apparent (heliocentric) rangerates were larger than $2 / 3$ of the maximum possible. In all other cases, the observed signals are attributed to both hemispheres (labelled LH, TH, or BH in column $\frac{\dot{m}_{\text {helio }}}{\left|\dot{r}_{\text {helio }}^{\text {max }}\right|}$ of Tables A.2-A.6).

\subsection{Oberon}

A standard radiometric analysis of the combined HerschelPACS, IRTF, and Spitzer-IRS measurements leads to a range of size-albedo-thermal solutions with reduced $\chi^{2}$-values close to or below 1.0. However, the optimum solutions resulted in an effective diameter which is about $3-5 \%$ above the object's true size (and a geometric albedo of 0.29 ), connected to a thermal inertia in the range $20-40 \mathrm{~J} \mathrm{~m}^{-2} \mathrm{~s}^{-0.5} \mathrm{~K}^{-1}$.

When we keep the diameter fixed to $1522 \mathrm{~km}$ (with $\mathrm{p}_{V}=0.31$ ) we can still find acceptable solutions (with reduced $\chi^{2}$-values close to 1): for an intermediate level of surface roughness (rms of surface slopes between 0.3 and 0.7 ) and thermal inertias between 9 and $33 \mathrm{~J} \mathrm{~m}^{-2} \mathrm{~s}^{-0.5} \mathrm{~K}^{-1}$ (higher thermal inertias are connected to higher levels of surface roughness and vice versa).

The best solution is found for a thermal inertia of around $20 \mathrm{~J} \mathrm{~m}^{-2} \mathrm{~s}^{-0.5} \mathrm{~K}^{-1}$ and an intermediate level of surface roughness $(\mathrm{rms}=0.5)$. We confirmed the solution by using a modified input data set where the close-proximity PACS data (at only 6 "apparent separation from Uranus) were eliminated.

The leading (PACS 2nd epoch, IRS-1)/trailing (PACS 4th and 5th epoch, IRS-2) analysis did not show any clear differences: both data subsets led to the same thermal properties (thermal inertia of $20 \mathrm{~J} \mathrm{~m}^{-2} \mathrm{~s}^{-0.5} \mathrm{~K}^{-1}$ ) with very similar reduced $\chi^{2}$ values. From the available measurements, we cannot distinguish the leading and trailing hemispheres. The IRS spectra confirm this finding: both spectra (in comparison with the corresponding optimum TPM prediction) agree within 5\%, except at the shortest end below $16 \mu \mathrm{m}$ where the difference is about $10 \%$.

The overall consistency $\left(\frac{f_{\text {moon,cc }}}{f_{\text {model }}}\right)$ of the models with colour corrected PACS fluxes for all five periods is $0.95 \pm 0.02$, $0.94 \pm 0.06$, and $1.00 \pm 0.02$ at 70,100 , and $160 \mu \mathrm{m}$, respectively. Excluding the first epoch photometry, where Oberon was at an only 6" apparent separation from Uranus, gives ratios of $0.95 \pm 0.01,0.96 \pm 0.01$, and $1.00 \pm 0.02$. An illustrated comparison for epoch 2 is shown in Fig. 7.

\subsection{Titania}

The standard thermal analysis (PACS, IRTF, IRS) led to reduced $\chi^{2}$ values close to 1 for a radiometric size which is again $2-4 \%$ larger than the true value, and a thermal inertia of 9$31 \mathrm{~J} \mathrm{~m}^{-2} \mathrm{~s}^{-0.5} \mathrm{~K}^{-1}$. All data are taken at sufficient separation from 


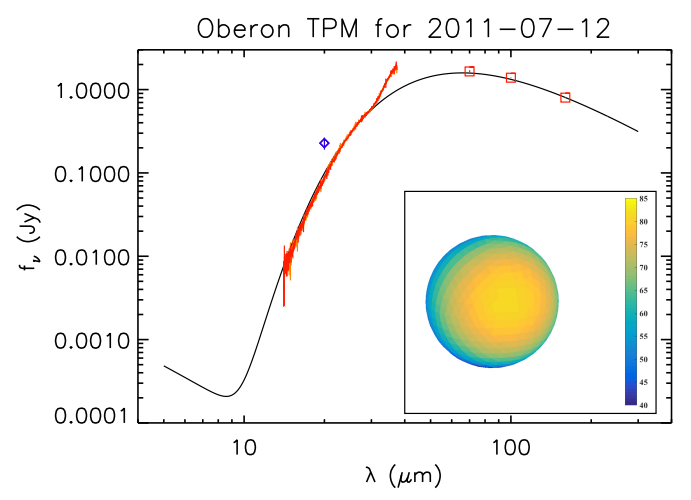

Fig. 7. Thermophysical model of Oberon between 5 and $300 \mu \mathrm{m}$ (black line) for the second epoch (2011-07-12). Photometric measurements are PACS observations (red boxes), the IRTF observation (blue diamond), and the Spitzer-IRS CASSIS data (orange spectrum: leading hemisphere observation; red spectrum: trailing hemisphere data). The CASSIS spectra suffer from Uranus stray light longward of $30 \mu \mathrm{m}$. The IRTF and IRS data were re-scaled to the model epoch with $\left(\frac{\Delta_{\mathrm{obs}, \text { IRS,IRTF }}}{\Delta_{\mathrm{obs}, 2011-07-12}}\right)^{2}$ Nevertheless, the IRTF flux appears to be too high with regard to the model, because the IRTF measurement was done under close to pole-on illumination, when the moon was hotter, while the model reflects more a viewing geometry close to equator-on, when the moon was colder due to heat transport to the night side. The insert shows the resulting TPM surface temperature map of Oberon for the range $40-85 \mathrm{~K}$.

Uranus ( $\left.>14^{\prime \prime}\right)$, but the IRS spectra still seem to be contaminated around $30 \mu \mathrm{m}$. Adding the constraints from Titania's known size and albedo (see Table 6), leads to thermal inertia values of 5$15 \mathrm{~J} \mathrm{~m}^{-2} \mathrm{~s}^{-0.5} \mathrm{~K}^{-1}$, with optimum values of 7-11 $\mathrm{J} \mathrm{m}^{-2} \mathrm{~s}^{-0.5} \mathrm{~K}^{-1}$, again for an intermediate level of surface roughness $(\mathrm{rms}=$ 0.4 ). We explicitly tested also other solutions for the thermal inertia, but a value of $20 \mathrm{~J} \mathrm{~m}^{-2} \mathrm{~s}^{-0.5} \mathrm{~K}^{-1}$, as found for Oberon, caused already severe problems in fitting our thermal measurements. The TPM predictions for the PACS measurements would decrease by $5-15 \%$ and the match to the observations would not be acceptable (outside 3- $\sigma$ ). The higher thermal inertia predictions would fit the IRS spectrum at short wavelength below $22 \mu \mathrm{m}$ and beyond $35 \mu \mathrm{m}$, but not in between. Overall, we can exclude a thermal inertia larger than about $15 \mathrm{~J} \mathrm{~m}^{-2} \mathrm{~s}^{-0.5} \mathrm{~K}^{-1}$ and smaller than about $5 \mathrm{~J} \mathrm{~m}^{-2} \mathrm{~s}^{-0.5} \mathrm{~K}^{-1}$ for Titania.

The Herschel-PACS measurements of Titania cover mainly the trailing hemisphere (1st, 2nd, and 5nd epoch) and a clean leading or trailing analysis is not possible. However, we ran our analysis on these trailing hemisphere measurements (three PACS epochs and IRS-2) and compared the results with the leading hemisphere IRS-1 measurement. The trailing data give a very consistent (reduced $\chi^{2}$ of 0.7 ) solution with a thermal inertia between 5 and $9 \mathrm{~J} \mathrm{~m}^{-2} \mathrm{~s}^{-0.5} \mathrm{~K}^{-1}$. But this solution overestimates the fluxes from the IRS-1 spectrum. A higher thermal inertia of 9-15 $\mathrm{J} \mathrm{m}^{-2} \mathrm{~s}^{-0.5} \mathrm{~K}^{-1}$ is needed to explain the leading hemisphere data. There are no PACS data to confirm this finding and due to the reduction and stray light residuals in the IRS spectra so close to Uranus; thus, this can only be considered an indication of differences between both hemispheres.

The overall consistency $\left(\frac{f_{\text {moon,ce }}}{f_{\text {model }}}\right)$ of the models with colour corrected PACS fluxes for all five epochs are $0.97 \pm 0.02$, $0.97 \pm 0.03$, and $0.99 \pm 0.03$ at 70,100 , and $160 \mu \mathrm{m}$, respectively. An illustrated comparison for epoch 2 is shown in Fig. 8.

\subsection{Umbriel}

The standard radiometric search for the object's best size, albedo, and thermal properties led to an unrealistically small thermal

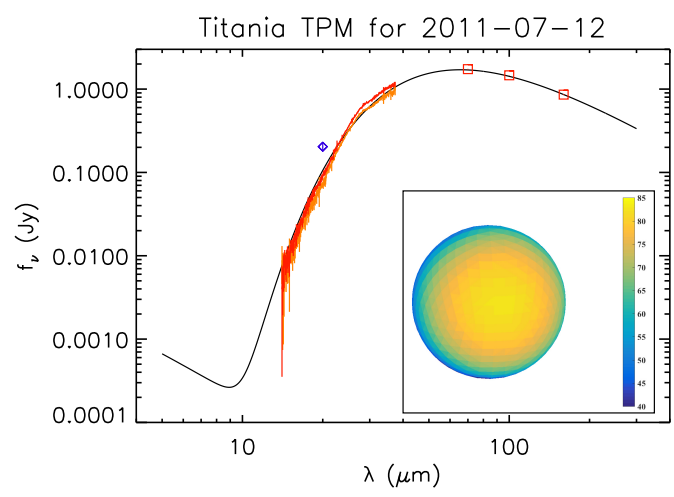

Fig. 8. Thermophysical model of Titania between 5 and $300 \mu \mathrm{m}$ (black line) for the second epoch (2011-07-12). Photometric measurements are PACS observations (red boxes), the IRTF observation (blue diamond) and the Spitzer-IRS CASSIS data (orange spectrum: leading hemisphere observation; red spectrum: trailing hemisphere data). For an explanation of the IRTF and IRS data re-scale, see text and caption of Fig. 7. The insert shows the resulting TPM surface temperature map of Titania for the range $40-85 \mathrm{~K}$.

inertia below $5 \mathrm{~J} \mathrm{~m}^{-2} \mathrm{~s}^{-0.5} \mathrm{~K}^{-1}$ and a diameter of just below $1100 \mathrm{~km}\left(p_{\mathrm{V}} \approx 0.30\right)$, with reduced $\chi^{2}$ values close to 1.0 . The size and albedo values are in clear contradiction to the published values of $1170 \mathrm{~km}$ and $p_{\mathrm{V}}=0.26$ (Karkoschka 2001). Taking the larger size requires a higher thermal inertia to fit all observed fluxes. Intermediate levels of surface roughness, combined with thermal inertias between 5 and $15 \mathrm{~J} \mathrm{~m}^{-2} \mathrm{~s}^{-0.5} \mathrm{~K}^{-1}$ seem to fit best (reduced $\chi^{2}$ values just below the 1.7 threshold).

However, if we look at the observation-to-model ratios we can identify a few observations which suffer from low signal-tonoise ratios (all five PACS measurements at $160 \mu \mathrm{m}$ and both IRS $15 \mu \mathrm{m}$ spectral parts have $S / N \leq 3$ ), but our radiometric weighted solutions handle correctly the proper flux errors. More problematic are the long-wavelengths fluxes when Umbriel had only a small apparent separation (below 7") from Uranus: the PACS 100 and $160 \mu \mathrm{m}$ measurements from 26-Dec.-2011 and also the long-wavelength parts of both IRS spectra beyond about $22 \mu \mathrm{m}$ seem to be affected by residual Uranus PSF features. Excluding these problematic measurements, we obtained reduced $\chi^{2}$-values close to 1.0 (for the fixed size of $1170 \mathrm{~km}$ ), with a preference for a lower surface roughness (around 0.3) than for Oberon and Titania, and a thermal inertia in the range between 5 and $12 \mathrm{~J} \mathrm{~m}^{-2} \mathrm{~s}^{-0.5} \mathrm{~K}^{-1}$.

The Umbriel data have a well-balanced coverage of the leading (PACS 1st epoch, IRS-1) and trailing (PACS 5th epoch, IRS-2) hemispheres. Separate fits to the data for the two hemispheres led to the following results: the fits to the trailing hemisphere data are excellent (reduced $\chi^{2}$ well below 1.0) with a thermal inertia at the lower end (around $5 \mathrm{~J} \mathrm{~m}^{-2} \mathrm{~s}^{-0.5} \mathrm{~K}^{-1}$ ). The leading hemisphere data show an indication for a slightly higher thermal inertia closer to $10 \mathrm{~J} \mathrm{~m}^{-2} \mathrm{~s}^{-0.5} \mathrm{~K}^{-1}$. However, within the error bars, both sets can be fit with an intermediate solution.

The overall consistency $\left(\frac{f_{\text {moon,ce }}}{f_{\text {model }}}\right)$ of the models with colour corrected PACS fluxes for all five epochs is $1.02 \pm 0.05$, $1.05 \pm 0.08$, and $1.16 \pm 0.12$ at 70,100 , and $160 \mu \mathrm{m}$, respectively. Excluding the second and third epoch, where Umbriel is at less than $9^{\prime \prime}$ apparent separation from Uranus, slightly improves the $70 \mu \mathrm{m}$ ratio $(1.01 \pm 0.04)$, but not the 100 and $160 \mu \mathrm{m}$ ratios $(1.05 \pm 0.08$ and $1.23 \pm 0.13$, respectively). An illustrated comparison for epoch 2 is shown in Fig. 9. 


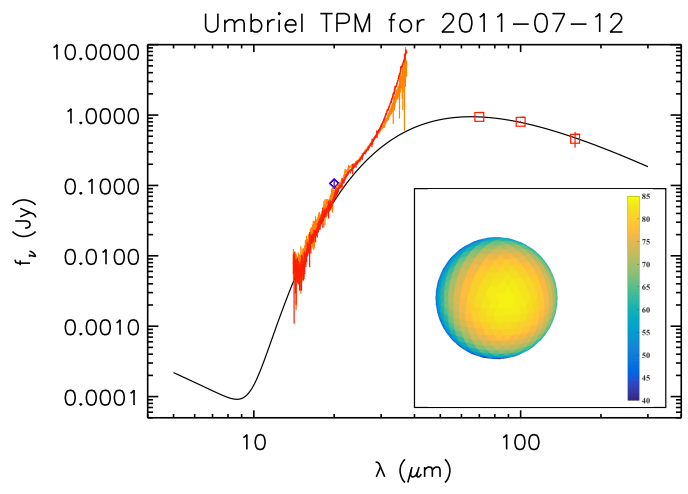

Fig. 9. Thermophysical model of Umbriel between 5 and $300 \mu \mathrm{m}$ (black line) for the second epoch (2011-07-12). Photometric measurements are PACS observations (red boxes), the IRTF observation (blue diamond), and the Spitzer-IRS CASSIS data (orange spectrum: leading hemisphere observation; red spectrum: trailing hemisphere data). The CASSIS spectra suffer from Uranus stray light longward of $22 \mu \mathrm{m}$. For an explanation of the IRTF and IRS data re-scale, see text and caption of Fig. 7. The insert shows the resulting TPM surface temperature map of Umbriel for the range 40-85 K.

\subsection{Ariel}

Ariel was also seen by IRTF, Spitzer-IRS (leading and trailing), and Herschel-PACS. However, the thermal IR fluxes are even lower than for Umbriel and the apparent distances to Uranus are smaller. Two PACS measurement sequences (08-Jun.-2012 and 14-Dec.-2012) were taken with Ariel below 6" separation and had to be skipped. None of the IRS spectra are usable: the fluxes are too high by factors of 3-45 (cf. Fig. 10).

A first radiometric analysis (just PACS $70 / 100 \mu \mathrm{m}$ fluxes and the IRTF flux) produced sizes between about 1100 and $1400 \mathrm{~km}$ and only a very weak constraint on the thermal inertia (values below $100 \mathrm{~J} \mathrm{~m}^{-2} \mathrm{~s}^{-0.5} \mathrm{~K}^{-1}$ ). Using the size constraint of $1159 \mathrm{~km}(a \times b: 581 \mathrm{~km} \times 578 \mathrm{~km}$, Karkoschka 2001) requires a thermal inertia between 6 and $25 \mathrm{~J} \mathrm{~m}^{-2} \mathrm{~s}^{-0.5} \mathrm{~K}^{-1}$ for an intermediate surface roughness. However, the reduced $\chi^{2}$ is larger than 2.0 and a closer inspection shows a clear separation in the fits to the leading and trailing hemispheres. Taking the PACS measurements for the leading hemisphere (2011-Jul.-12) and the trailing hemisphere (2010-Dec.-13, and 2011-Dec.-26) separately gives much better fits (reduced $\chi^{2}$ close to 1.0), indicating a lower thermal inertia $\left(5-13 \mathrm{~J} \mathrm{~m}^{-2} \mathrm{~s}^{-0.5} \mathrm{~K}^{-1}\right)$ for the leading hemisphere, and a higher thermal inertia $\left(13-40 \mathrm{~J} \mathrm{~m}^{-2} \mathrm{~s}^{-0.5} \mathrm{~K}^{-1}\right.$ ) for the trailing hemisphere. Although the IRS spectra cannot be used for the radiometric studies, the flux levels for the leading hemisphere are about $5-10 \%$ higher. This also points to a lower thermal inertia for the leading side compared to the trailing side. With our final solution, we calculated a maximum brightness temperature of about $86 \mathrm{~K}$ for the South-pole viewing geometry in early 1986. This compares very well with the maximum brightness temperature of $84 \pm 1 \mathrm{~K}$ seen by Voyager-2/IRIS (Hanel et al. 1986).

The overall consistency $\left(\frac{f_{\text {moon,cc }}}{f_{\text {model }}}\right)$ of the models with colour corrected PACS fluxes for all five epochs is $1.07 \pm 0.12$, $0.94 \pm 0.29$, and $2.09 \pm 0.48$ at 70,100 , and $160 \mu \mathrm{m}$, respectively. The high $160 \mu \mathrm{m}$ ratio of $\gtrsim 2$ is due to the fact that the measured values are all, except for one, far too low. Excluding the fourth and fifth epoch, where Ariel is at less than 6"apparent separation from Uranus, improves the consistency at 70 and $100 \mu \mathrm{m}$ considerably with ratios of $0.99 \pm 0.04,1.00 \pm 0.17$, respectively. However, due to the generally low $160 \mu \mathrm{m}$ fluxes, this ratio $(2.20 \pm 0.11)$ does not improve. An illustrated comparison for epoch 2 is shown in Fig. 10.

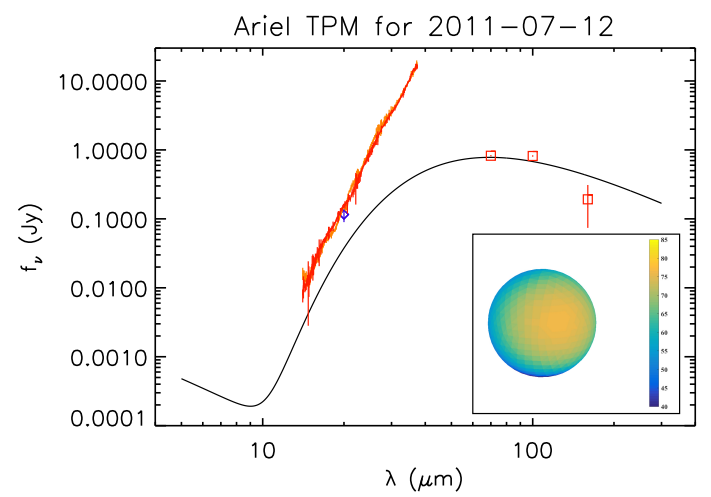

Fig. 10. Thermophysical model of Ariel between 5 and $300 \mu$ m (black line) for the second epoch (2011-07-12). Photometric measurements are PACS observations (red boxes), the IRTF observation (blue diamond). and the Spitzer-IRS CASSIS data (orange spectrum: leading hemisphere observation; red spectrum: trailing hemisphere data). The CASSIS spectra suffer from Uranus stray light over their full wavelength range. For an explanation of the IRTF and IRS data re-scale, see the text and caption of Fig. 7. The insert shows the resulting TPM surface temperature map of Ariel for the range between $40-85 \mathrm{~K}$.

\subsection{Miranda}

For Miranda we have only the PACS measurements, but neither IRTF nor Spitzer-IRS data. The object was always within $10^{\prime \prime}$ from Uranus and the contamination problems are severe. We eliminated all $160 \mu \mathrm{m}$ fluxes which are clearly completely off. In addition, we skipped the second- and fourth-epoch data, when Miranda was only at 3.'4 and 4". 4 apparent distance, respectively. For the last epoch, we also had to take out the $100 \mu \mathrm{m}$ data.

In the end, only very few data points remained and the required coverage (in aspect angles, wavelengths, leading and trailing geometries, etc.) is missing for a robust radiometric analysis. With the size $(474 \mathrm{~km})$ and albedo $\left(p_{\mathrm{V}}=0.45\right)$ we only obtained an upper limit of about $50 \mathrm{~J} \mathrm{~m}^{-2} \mathrm{~s}^{-0.5} \mathrm{~K}^{-1}$ for Miranda's thermal inertia. Larger values would force the TPM calculations to smaller fluxes which are not compatible with the highest $\mathrm{S} / \mathrm{N}$ detections by PACS (the upper limit goes down to $20 \mathrm{~J} \mathrm{~m}^{-2} \mathrm{~s}^{-0.5} \mathrm{~K}^{-1}$, if we consider only the best $70 \mu \mathrm{m}$ fluxes). The corresponding TPM calculations (with a thermal inertia below $20 \mathrm{~J} \mathrm{~m}^{-2} \mathrm{~s}^{-0.5} \mathrm{~K}^{-1}$ ) for the Voyager-2/IRIS measurements in January 1986 produce a maximum temperature of about $87 \mathrm{~K}$, in excellent agreement with the $86 \pm 1 \mathrm{~K}$ by Hanel et al. (1986).

\subsection{Discussion}

Table 7 provides an overview of the derived model parameters. Using these model SEDs, we also calculated the colour correction factors to be applied to the measured PACS fluxes (cf. Tables A.2-A.6).

How do the derived properties for the Uranian satellites compare with thermal inertias of other satellites and distant trans-neptunian objects (TNOs)? Lellouch et al. (2013) analysed a large sample of TNOs and found a $\Gamma=2.5 \pm 0.5 \mathrm{~J} \mathrm{~m}^{-2} \mathrm{~s}^{-1 / 2} \mathrm{~K}^{-1}$ for objects at heliocentric distances of $r_{\text {helio }}=20-50 \mathrm{AU}$ (decreasing values for increasing heliocentric distance). The Uranian system is at about $20 \mathrm{AU}$ and therefore one would expect (under the assumption of TNO-like surfaces) to find low values, maybe up to $5 \mathrm{~J} \mathrm{~m}^{-2} \mathrm{~s}^{-1 / 2} \mathrm{~K}^{-1}$.

However, looking at dwarf planets, these general TNOderived values are usually exceeded: Haumea is at $\mathrm{r}_{\text {helio }}=\sim 51 \mathrm{AU}$ and it was found to have a thermal inertia of around $10 \mathrm{~J} \mathrm{~m}^{-2} \mathrm{~s}^{-1 / 2} \mathrm{~K}^{-1}$ (Müller et al. 2019). The thermal inertias 
A\&A 641, A76 (2020)

Table 7. Overview of the best thermophysical model parameter ranges and the resulting PACS filter colour correction factors cc.

\begin{tabular}{lccccc}
\hline \hline Object & $\begin{array}{c}\Gamma \\
\left(\mathrm{J} \mathrm{m}^{-2} \mathrm{~s}^{-0.5} \mathrm{~K}^{-1}\right)\end{array}$ & $\begin{array}{c}\text { Surface roughness } \\
(\mathrm{rms})\end{array}$ & $\mathrm{cc}_{70}$ & $\mathrm{cc}_{100}$ & $\mathrm{cc}_{160}$ \\
\hline Titania (UIII) [703] & $7-11$ & 0.4 & 0.984 & 0.999 & 1.032 \\
Oberon (UIV) [704] & 20 & 0.5 & 0.984 & 0.999 & 1.032 \\
Umbriel (UII) [702] & $5-12$ & 0.3 & 0.984 & 0.999 & 1.032 \\
Ariel (UI) [701] & $5-13(\mathrm{LH}), 13-40(\mathrm{TH})$ & 0.5 & 0.983 & 0.997 & 1.030 \\
Miranda (UV) [705] & $<20$ & 0.5 & 0.983 & 0.998 & 1.030 \\
\hline
\end{tabular}

of Pluto and Charon (at $r_{\text {helio }}>30 \mathrm{AU}$ ) are even larger: $\Gamma_{\text {Pluto }}=$ $16-26 \mathrm{~J} \mathrm{~m}^{-2} \mathrm{~s}^{-1 / 2} \mathrm{~K}^{-1}$ and $\Gamma_{\text {Charon }}=9-14 \mathrm{~J} \mathrm{~m}^{-2} \mathrm{~s}^{-1 / 2} \mathrm{~K}^{-1}$ (Lellouch et al. 2011, 2016). And putting the Pluto-Charon system closer to the Sun would increase the values significantly (assuming that the $\mathrm{T}^{3}$ term dominates in the thermal conductivity, then the thermal inertia scales with $\propto r^{-3 / 4}$; see e.g. Delbo et al. 2015). In case of Pluto-Charon, the high $\Gamma$-values are attributed to a large diurnal skin depth due to their slow rotation $\left(\sim \mathrm{P}^{1 / 2}\right.$ dependence; see also discussion in Kiss et al. 2019). In summary, the Uranian satellites Oberon, Titania, Umbriel, Ariel, and Miranda have thermal inertias which are higher than the very low values found for TNOs and Centaurs at $30 \mathrm{AU}$ heliocentric distance. It seems that the thermal properties of the icy satellite surfaces are closer to the properties found for the TNO dwarf planets Pluto and Haumea.

\section{Conclusions}

In this study, we successfully demonstrate an image processing technique for PACS photometer data, allowing us to remove the bright central PSF of Uranus and reconstructing source fluxes of its five major satellites on the order of $10^{-3}$ of Uranus. We obtained reliable moon fluxes outside radii of $7 . .8,11^{\prime \prime} 1$, and 17 '. 8 at 70,100 , and $160 \mu \mathrm{m}$, respectively, which corresponds to $\approx 3 \times$ the half width at half maximum of the standard PSF $\left(F W H M_{\mathrm{PSF}}=5^{\prime \prime}\right.$.6, 6.' 8 and 10.'7, respectively $)$. For Titania and Oberon we have established full sets of 70, 100, and $160 \mu \mathrm{m}$ PSF photometry for all five observing epochs. For Umbriel, there are two epochs $(1 \& 5)$ with high quality 70 and $100 \mu$ m photometry and for Ariel, there are three epochs (1-3). The $160 \mu \mathrm{m}$ photometry of these two moons is either of low quality (Umbriel) or unreliable (Ariel). For Miranda, $70 \mu \mathrm{m}$ flux estimates could be obtained for two epochs ( $1 \& 3)$. This new FIR photometry, in combination with auxiliary data at shorter wavelengths from literature, has allowed us to establish TPM solutions for the five major Uranus satellites and to put constraints on their thermal inertia and surface roughness.

Acknowledgements.PACS has been developed by a consortium of institutes led by MPE (Germany) and including UVIE (Austria); KUL, CSL, IMEC (Belgium); CEA, OAMP (France); MPIA (Germany); IFSI, OAP/AOT, OAA/CAISMI, LENS, SISSA (Italy); IAC (Spain). This development has been supported by the funding agencies BMVIT (Austria), ESA-PRODEX (Belgium), CEA/CNES (France), DLR (Germany), ASI (Italy), and CICYT/MCYT (Spain). Z.B acknowledges funding by DLR for this work. T.M. has received funding from the European Union's Horizon 2020 Research and Innovation Programme, under Grant Agreement no. 687378, as part of the project "Small Bodies Near and Far" (SBNAF). We have made use of the JPL Horizons On-Line Ephemeris System to derive orbital parameters of the Uranian moons. The Combined Atlas of Sources with Spitzer IRS Spectra (CASSIS) is a product of the IRS instrument team, supported by NASA and JPL. CASSIS is supported by the "Programme National de Physique Stellaire" (PNPS) of CNRS/INSU co-funded by CEA and CNES and through the "Programme National Physique et Chimie du Milieu Interstellaire"
(PCMI) of CNRS/INSU with INC/INP co-funded by CEA and CNES. GM was supported by the Hungarian National Research, Development and Innovation Office (NKFIH) grant PD-128360. G.M. acknowledges partial support from the EC Horizon 2020 project OPTICON (730890) and the ESA PRODEX contract nr. 4000129910 . We thank the referee for constructive comments.

\section{References}

Balog, Z., Müller, T., Nielbock, M., et al. 2014, Exp. Astron., 37, 129 Brown, R. H., Cruikshank, D. P., \& Morrison, D. 1982, Nature, 300, 423 Cartwright, R. J., Emery, J. P., Rivkin, A. S., Trilling, D. E., \& Pinilla-Alonso, N. 2015, Icarus, 257, 428

Cartwright, R. J., Emery, J. P., Pinilla-Alonso, N., et al. 2018, Icarus, 314, 210

Chapin, E. L., Berry, D. S., Gibb, A. G., et al. 2013, MNRAS, 430, 2545

Cohen, M., Witteborn, F. C., Carbon, D. F., et al. 1996, AJ, 112, 2274

Delbo, M., Mueller, M., Emery, J. P., Rozitis, B., \& Capria, M. T. 2015, Asteroids IV (Tucson, AZ: University of Arizona Press), 107

Exter, K., Balog, Z., Calzoletti, L., et al. 2018, The Photodetector Array Camera and Spectrometer (PACS) Handbook, Herschel-HSC-DOC-2101, https://www. cosmos. esa.int/documents/12133/996891/PACS+ Explanatory+Supplement

Fruchter, A. S., \& Hook, R. N. 2002, PASP, 114, 144

Hanel, R., Conrath, B., Flasar, F. M., et al. 1986, Science, 233, 70

Harper, D. A., Runyan, M. C., Dowell, C. D., et al. 2018, J. Astron. Instrum., 7, 1840008

Houck, J. R., Roellig, T. L., van Cleve, J., et al. 2004, ApJS, 154, 18

Karkoschka, E. 2001, Icarus, 151, 51

Kiss, C., Marton, G., Parker, A. H., et al. 2019, Icarus, 334, 3

Klaas, U. 2016, Herschel Technical Report on Herschel telescope background in photometer bands, PICC-MA-TN-013, https://www. cosmos.esa.int/ documents/12133/996891/A+Herschel+telescope+background+ model+for+the+PACS+photometer

Kramer, C., Moreno, R., \& Greve, A. 2008, A\&A, 482, 359

Lagerros, J. S. V. 1996, A\&A, 310, 1011

Lagerros, J. S. V. 1997, A\&A, 325, 1226

Lagerros, J. S. V. 1998, A\&A, 332, 1123

Lebouteiller, V., Barry, D. J., Spoon, H. W. W., et al. 2011, ApJS, 196, 8

Lebouteiller, V., Barry, D. J., Goes, C., et al. 2015, ApJS, 218, 21

Lellouch, E., Stansberry, J., Emery, J., Grundy, W., \& Cruikshank, D. P. 2011, Icarus, 214, 701

Lellouch, E., Santos-Sanz, P., Lacerda, P., et al. 2013, A\&A, 557, A60

Lellouch, E., Santos-Sanz, P., Fornasier, S., et al. 2016, A\&A, 588, A2

Lemke, D., Klaas, U., Abolins, J., et al. 1996, A\&A, 315, L64

Lutz, D. 2015, Herschel Technical Report on PACS photometer point spread function, PICC-ME-TN-033, https://www. cosmos.esa.int/ documents/12133/996891/PACS+photometer+point+spread+ function

Morrison, D., \& Lebofsky, L. 1979, Asteroids, eds. T. Gehrels, \& M. S. Matthews (Tucson, AZ: University of Arizona Press), 184

Müller, T. G., \& Lagerros, J. S. V. 1998, A\&A, 338, 340

Müller, T. G., \& Lagerros, J. S. V. 2002, A\&A, 381, 324

Müller, T. G., Balog, Z., Nielbock, M., et al. 2016, A\&A, 588, A109

Müller, T. G., Kiss, C., Ali-Lagoa, V., et al. 2019, Icarus, 334, 39

Ott, S. 2010, ASP Conf. Ser., 434, 139

Pilbratt, G., Riedinger, J., Passvogel, T., et al. 2010, A\&A, 518, L1

Poglitsch, A., Waelkens, C., Geis, N., et al. 2010, A\&A, 518, id.L2

Szakáts, R., Müller, T., Alí-Lagoa, V., et al. 2020, A\&A, 635, A54

Veverka, J., Brown, R. H., \& Bell, J. F. 1991, Uranus satellites - Surface properties, eds. J. T. Bergstralh, E. D. Miner, \& M. S. Matthews (Tucson, AZ: University of Arizona Press), 528 


\section{Appendix A: PSF subtracted maps and photometry of individual maps}

\section{A.1. $70 \mu \mathrm{m}$ maps of Uranian moons}
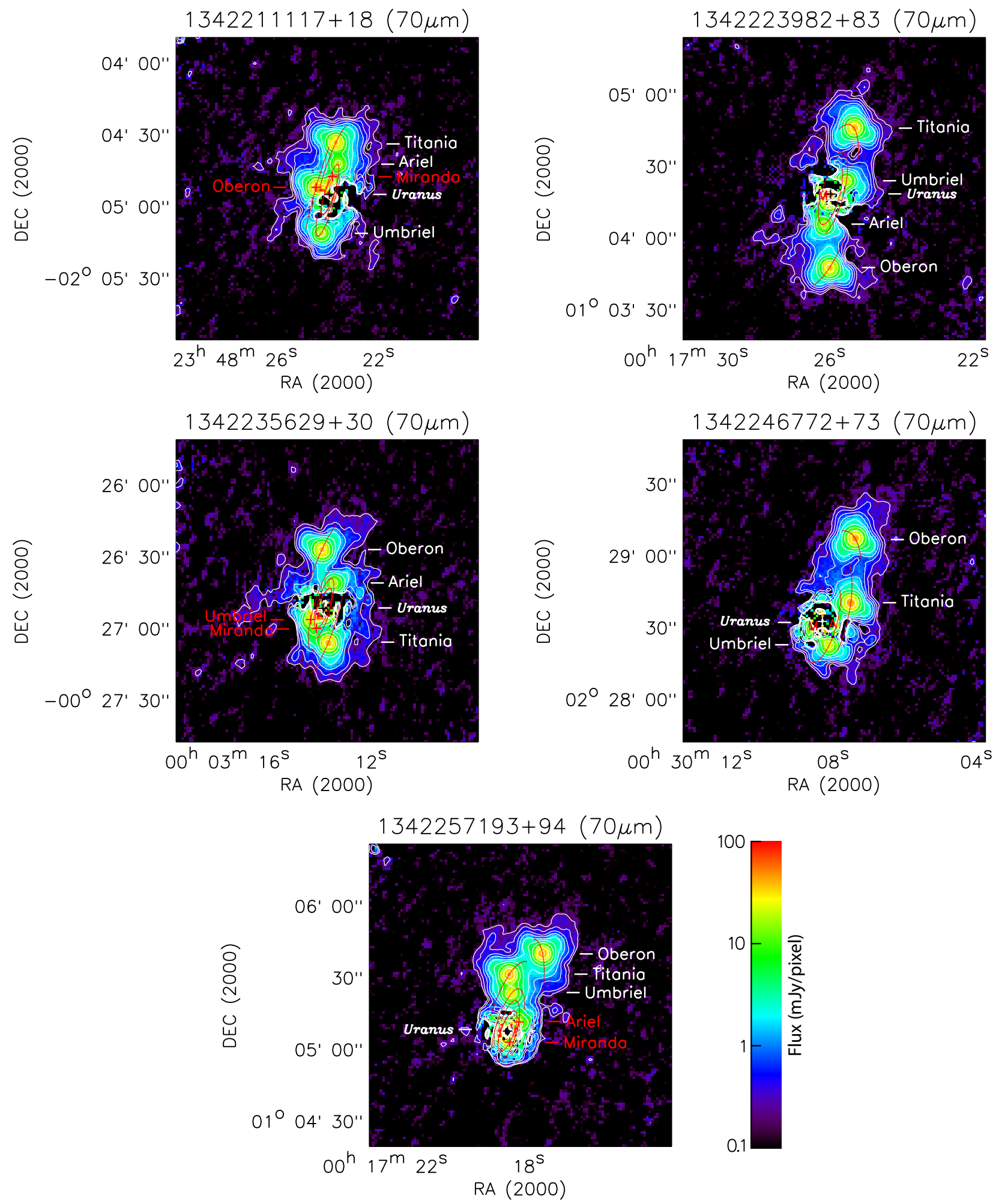

Fig. A.1. $70 \mu \mathrm{m}$ maps of the Uranus moons after subtraction of the Uranus PSF reference. Pixel scale is 1". 1 . Moons for which the PSF is not or only slightly affected by PSF subtraction residuals are labelled in white. The central positions of moons for which the PSF is more significantly affected by PSF subtraction residuals or which are located closely together are marked by a red cross. If only the initial of a moon is labelled, then its PSF peak is located inside the critical residual area. The central position of Uranus is marked by a black or white cross and labelled in italics. The positions of the moons relative to Uranus within plus minus one day of the observation are indicated by small red lines (we note that these refer to the Uranus position at the time of observation). Since Miranda has an orbital period of $P=1.413 \mathrm{~d}$, a closed orbit line is seen. 


\section{A.2. $100 \mu \mathrm{m}$ maps of Uranian moons}
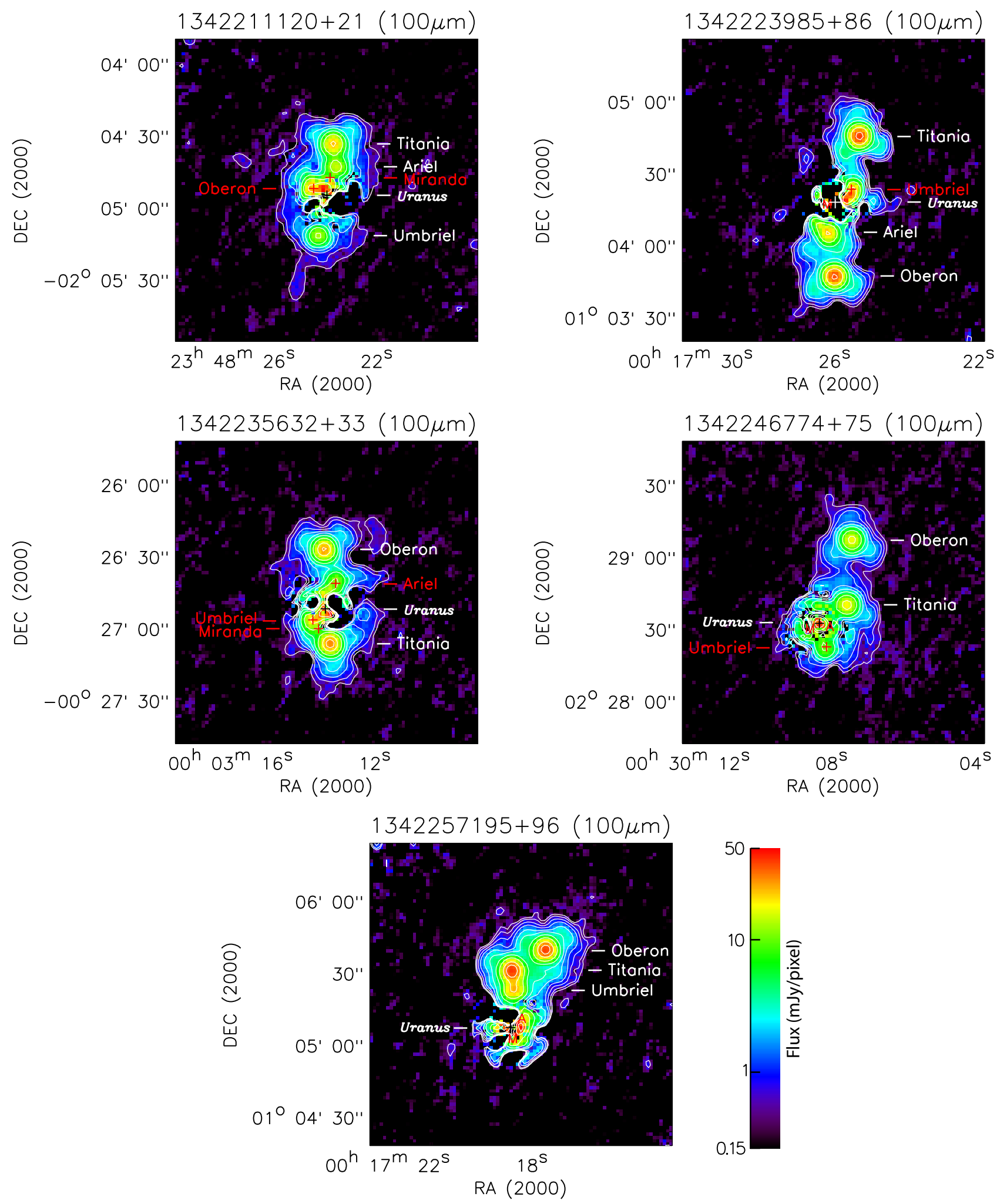

Fig. A.2. $100 \mu \mathrm{m}$ maps of the Uranus moons after subtraction of the Uranus PSF reference. Pixel scale is 1". 4 . Moons for which the PSF is not or only slightly affected by PSF subtraction residuals are labelled in white. The central positions of moons for which the PSF is more significantly affected by PSF subtraction residuals or which are located closely together are marked by a red cross. If only the initial of a moon is labelled, then its PSF peak is located inside the critical residual area. The central position of Uranus is marked by a black or white cross and labelled in italics. 


\section{A.3. $160 \mu m$ maps of Uranian moons}
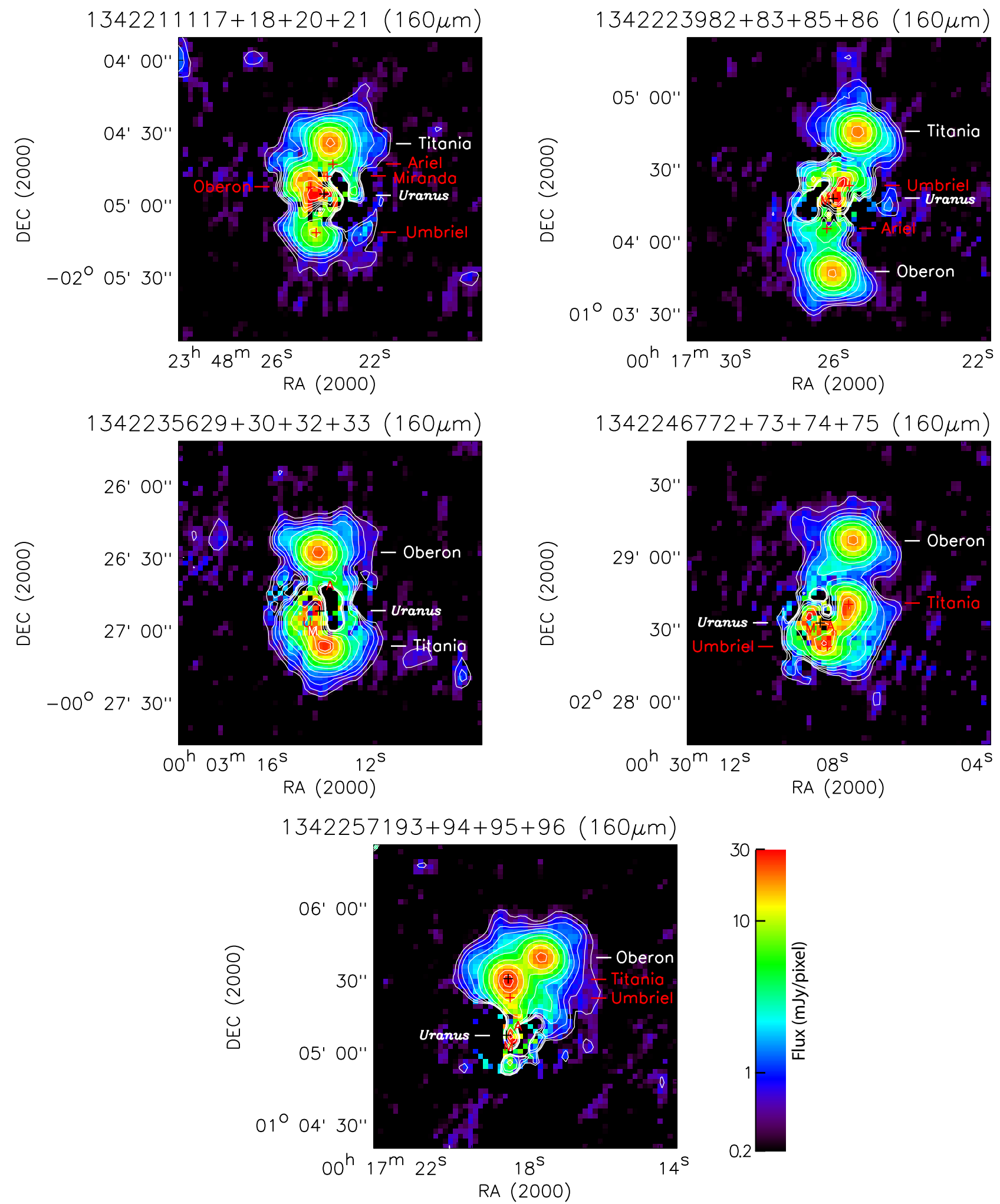

Fig. A.3. $160 \mu \mathrm{m}$ maps of the Uranus moons after subtraction of the Uranus PSF reference. Pixel scale is $22^{\prime \prime} 1$. Moons for which the PSF is not or only slightly affected by PSF subtraction residuals are labelled in white. The red cross marks the central positions of moons for which the PSF is more significantly affected by PSF subtraction residuals or which are located closely together are marked. If only the initial of a moon is labelled, then its PSF peak is located inside the critical residual area. The central position of Uranus is marked by a black or white cross and labelled in italics. 
A.4. Photometry of Uranus from individual maps

Table A.1. Photometry of Uranus.

\begin{tabular}{|c|c|c|c|c|c|c|c|c|c|c|c|}
\hline OBSID & OD & $\begin{array}{c}\text { MJD } \\
\text { mid-time obs. }\end{array}$ & $\begin{array}{l}\lambda_{\mathrm{ref}} \\
\mu \mathrm{m}\end{array}$ & $\begin{array}{l}f_{\text {total }} \\
(\mathrm{Jy})\end{array}$ & $\begin{array}{c}f_{\text {Uranus }}^{\text {measured }} \\
(\mathrm{Jy})\end{array}$ & $\begin{array}{l}\sigma_{\text {par }} \\
(\mathrm{Jy})\end{array}$ & $\begin{array}{l}\sigma_{\text {red }} \\
(\mathrm{Jy})\end{array}$ & $\begin{array}{l}\sigma_{\text {tot }} \\
(\mathrm{Jy})\end{array}$ & $\begin{array}{c}f_{\text {Uranus }}^{\text {distancecorrected }} \\
\text { (Jy) }\end{array}$ & $\begin{array}{l}r_{\text {helio }} \\
(\mathrm{AU})\end{array}$ & $\begin{array}{c}\Delta_{\text {obs }} \\
(\mathrm{AU})\end{array}$ \\
\hline \multirow[t]{2}{*}{1342211117} & 579 & 55543.72060 & 70.0 & 5 & 6 & 6 & 35 & 32 & & 20.090 & 19.975 \\
\hline & & & 160.0 & 667 & 665.241 & 0.420 & 0.453 & 0.617 & 7 & & \\
\hline \multirow[t]{2}{*}{1342211118} & 579 & 55543.72464 & 70.0 & 869.527 & 864.426 & 0.321 & 1.072 & 1.119 & 860 & 20.090 & 19.975 \\
\hline & & & 160.0 & 670 & 668.005 & 0.467 & 0.505 & 0.688 & 66 & & \\
\hline \multirow[t]{2}{*}{1342211120} & 579 & 55543.73128 & 100.0 & 888.362 & 884.066 & 0.317 & 0.354 & 0.475 & & 20.090 & 19.975 \\
\hline & & & 160.0 & 669.061 & 666.696 & 0.415 & 0.411 & 0.584 & 663.668 & & \\
\hline \multirow[t]{2}{*}{1342211121} & 579 & 55543.73532 & 100.0 & 887.348 & 882.873 & 0.322 & 0.302 & 0.441 & 878.867 & 20.090 & 19.975 \\
\hline & & & 160.0 & 670.997 & 668.587 & 0.475 & 0.742 & 0.881 & 665.555 & & \\
\hline \multirow[t]{2}{*}{1342223982} & 789 & 55754.05794 & 70.0 & 886 & 881. & 0.366 & 0.360 & .513 & 39 & 20.083 & 19.801 \\
\hline & & & 160.0 & & & 97 & & & & & \\
\hline \multirow[t]{2}{*}{1342223983} & 789 & 55754.06198 & 70.0 & 882.336 & 877.252 & 0.377 & 0.338 & 0.540 & & 20.083 & 19.801 \\
\hline & & & 160.0 & 681.133 & 678.773 & 0.432 & 0.380 & 0.575 & 82 & & \\
\hline \multirow[t]{2}{*}{1342223985} & 789 & 55754.06862 & 100.0 & 905.048 & 900.614 & 0.321 & 0.433 & 0.539 & 77 & 20.083 & 19.801 \\
\hline & & & 160.0 & & & 0.416 & 09 & 30 & & & \\
\hline \multirow[t]{2}{*}{1342223986} & 789 & 55754.07266 & 100.0 & 901.061 & 896.569 & 0.299 & 0.588 & 0.656 & 877.015 & 20.083 & 19.801 \\
\hline & & & 160.0 & 683.285 & 680.924 & 0.442 & 0.291 & 0.529 & 666.075 & & \\
\hline \multirow[t]{2}{*}{1342235629} & 957 & 55921 & 70.0 & & & & & & & 20.076 & 20.118 \\
\hline & & & 160.0 & & & 04 & 91 & 36 & & & \\
\hline \multirow[t]{2}{*}{1342235630} & 957 & 55921.95328 & 70.0 & 856.286 & 851. & 0.371 & 1.424 & 1.472 & 599 & 20.076 & 20.118 \\
\hline & & & 160.0 & & & 0.441 & 0.322 & & & & \\
\hline \multirow[t]{2}{*}{1342235632} & 957 & 55921.95992 & 100.0 & 31 & 87 & 0.307 & 0.405 & 09 & 540 & 20.076 & 20.118 \\
\hline & & & 160.0 & 20 & & 28 & 0.672 & 96 & 60 & & \\
\hline \multirow[t]{2}{*}{1342235633} & 957 & 55921.96396 & 100.0 & 872.182 & 867.978 & 0.318 & 0.512 & 0.603 & 876.465 & 20.076 & 20.118 \\
\hline & & & 160.0 & 661.010 & 658.770 & 0.470 & 0.366 & 0.596 & 665.212 & & \\
\hline \multirow[t]{2}{*}{1342246772} & 1121 & 56086.18500 & 70.0 & & & 4 & 10 & 50 & & 20.069 & 20.404 \\
\hline & & & 160.0 & & & & & & & & \\
\hline \multirow[t]{2}{*}{1342246773} & 1121 & 56086.18904 & 70.0 & 833.527 & 828.737 & 0.392 & 1.504 & 1.554 & 819 & 20.069 & 20.404 \\
\hline & & & 160.0 & 640.467 & 63 & 26 & 0.900 & 0.996 & 34 & & \\
\hline \multirow[t]{2}{*}{1342246774} & 1121 & 56086.19308 & 100.0 & 851.492 & 847.114 & 0.337 & 0.207 & 0.395 & 879.898 & 20.069 & 20.404 \\
\hline & & & & & & 0.413 & 0.709 & 0.820 & 662.352 & & \\
\hline \multirow[t]{2}{*}{1342246775} & 1121 & 56086.19712 & 100.0 & 849.254 & 844.839 & 0.332 & 0.554 & 0.646 & 877.528 & 20.069 & 20.404 \\
\hline & & & 160.0 & 640.252 & 637.971 & 0.482 & 0.340 & 0.590 & 662.657 & & \\
\hline \multirow[t]{2}{*}{1342257193} & 1310 & 56275.07361 & 70.0 & & & & 10 & & & 20.059 & 19.823 \\
\hline & & & 160.0 & & & & & & & & \\
\hline \multirow[t]{2}{*}{1342257194} & 1310 & 56275.07765 & 70.0 & 880.011 & 874.862 & 0.360 & 1.234 & 1.286 & 857.635 & 20.059 & 19.823 \\
\hline & & & 160.0 & 681.238 & 678.930 & 0.427 & 0.644 & 0.773 & 665.561 & & \\
\hline \multirow[t]{2}{*}{1342257195} & 1310 & 56275.08169 & 100.0 & 902.984 & 898.793 & 0.296 & 0.459 & 0.546 & 881.098 & 20.059 & 19.823 \\
\hline & & & & & & & & 0.619 & & & \\
\hline \multirow[t]{2}{*}{1342257196} & 1310 & 56275.08573 & 100.0 & 899.319 & 894.907 & 0.321 & 0.478 & 0.576 & 877.294 & 20.059 & 19.823 \\
\hline & & & 160.0 & 680.801 & 678.403 & 0.458 & 0.556 & 0.720 & 665.053 & & \\
\hline
\end{tabular}

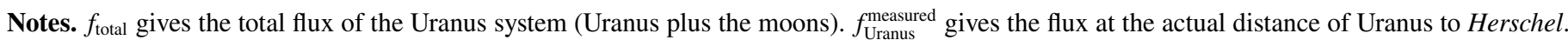
$f_{\text {Uranus }}^{\text {distancecorrected }}$ gives the flux corrected to the same mean distance $\left(\Delta_{\text {obs,mean }}=20.024 \mathrm{AU}\right)$ by scaling $f_{\text {Uranus }}^{\text {distancecorrected }}=\left(\frac{\Delta_{\text {obs }}}{\Delta_{\text {obs,mean }}}\right)^{2} \times f_{\text {Uranus }}^{\text {measured }} . \Delta_{\text {obs }}$ is the range of target centre wrt. the observer, i.e. Herschel, $r_{\text {helio }}$ is the light-time corrected heliocentric range. $\sigma_{\text {par }}$ is the uncertainty in the PSF fitting parameters, $\sigma_{\text {red }}$ is the uncertainty due to the reduction method (dependence on the map parameter selection), $\sigma_{\text {tot }}$ is the geometrical mean of the latter two uncertainties. The five epochs with 4 observations each are separated by horizontal lines. 


\section{A.5. PSF photometry of moons from individual maps}

Table A.2. PSF photometry of Titania.

\begin{tabular}{|c|c|c|c|c|c|c|c|c|c|c|c|c|c|c|c|c|}
\hline OBSID & OD & $\begin{array}{c}\text { MJD } \\
\text { mid-time obs. }\end{array}$ & $\begin{array}{l}\lambda_{\mathrm{ref}} \\
\mu \mathrm{m}\end{array}$ & $\begin{array}{l}\frac{f_{\text {moon }}}{f_{\text {Uranus }}} \\
\left(10^{-3}\right)\end{array}$ & $\begin{array}{c}f_{\text {moon }} \\
(\mathrm{Jy})\end{array}$ & $\begin{array}{l}\sigma_{\mathrm{par}} \\
(\mathrm{Jy})\end{array}$ & $\begin{array}{r}\sigma_{\text {red }} \\
(\mathrm{Jy})\end{array}$ & $\begin{array}{l}\sigma_{\text {tot }} \\
(\mathrm{Jy})\end{array}$ & $\begin{array}{c}f_{\text {moon,cc }} \\
(\mathrm{Jy})\end{array}$ & $\begin{array}{c}f_{\text {model }} \\
(\mathrm{Jy})\end{array}$ & $\frac{f_{\text {moon,cc }}}{f_{\text {model }}}$ & $\begin{array}{l}r_{\text {helio }} \\
\text { (AU) }\end{array}$ & $\begin{array}{l}\Delta_{\mathrm{obs}} \\
(\mathrm{AU})\end{array}$ & $\begin{array}{c}\alpha \\
(\operatorname{deg})\end{array}$ & $\begin{array}{c}\theta_{\mathrm{U}-\mathrm{O}} \\
\left({ }^{\prime \prime}\right)\end{array}$ & $\frac{\dot{r}_{\text {helio }}}{\left|\dot{r}_{\text {helia }}^{\text {hax }}\right|}$ \\
\hline \multirow[t]{2}{*}{1342211117} & 579 & 55543.72060 & 70.0 & 1.926 & 1.669 & 0.0011 & 0.0164 & 0.0165 & 1.696 & 1.650 & 1.028 & 38 & 73 & 26 & 21.86 & +0.7 \\
\hline & & & 160.0 & 1.315 & 0.875 & 0.0061 & 0.0094 & 0.0112 & 0.848 & 0.839 & 1.010 & & & & & \\
\hline \multirow[t]{2}{*}{1342211118} & 579 & 55543.72464 & 70.0 & 1.943 & 1.680 & 0.0011 & 0.0181 & 0.0181 & 1.707 & 1.650 & 1.034 & 20.088 & 19.973 & 2.826 & 21.92 & -0. \\
\hline & & & 160.0 & 1.282 & 0.856 & 0.0061 & 0.0149 & 0.0161 & 0.830 & 0.839 & 0.989 & & & & & \\
\hline \multirow[t]{2}{*}{1342211120} & 579 & 55543.73128 & 100.0 & 1.597 & 1.412 & 0.0018 & 0.0058 & 0.0061 & 1.413 & 1.385 & 1.020 & 20.088 & 19.973 & L 2.826 & 22.01 & $+0.72(\mathrm{TH})$ \\
\hline & & & 160.0 & 1.296 & 0.864 & 0.0060 & 0.0082 & 0.0102 & 0.837 & 0.839 & 0.998 & & & & & \\
\hline \multirow[t]{2}{*}{1342211121} & 579 & 55543.73532 & 100.0 & 1.583 & 1.398 & 0.0019 & 0.0056 & 0.0059 & 1.399 & 1.385 & 1.010 & 20.088 & 19.973 & L 2.826 & 22.07 & +0.7 \\
\hline & & & 160.0 & 1.277 & 0.854 & 0.0058 & 0.0125 & 0.0138 & 0.827 & 0.839 & 0.986 & & & & & \\
\hline \multirow[t]{2}{*}{1342223982} & 789 & 5575 & 70.0 & 1.934 & 1.705 & 0.0012 & 0.0151 & 0.0152 & 1.732 & 1.697 & 1.021 & 20.084 & 19.802 & Т 2.837 & 29.52 & +0. \\
\hline & & & 160.0 & 1.307 & 0.884 & 0.0031 & 0.0087 & 0.0093 & 0.856 & 0.860 & 0.996 & & & & & \\
\hline \multirow[t]{2}{*}{1342223983} & 789 & 55754.06198 & 70.0 & 1.949 & 1.710 & 0.0012 & 0.0163 & 0.0164 & 1.737 & 1.697 & 1.024 & 20.084 & 19.802 & Т 2.837 & 29.50 & $+0.97(\mathrm{TH})$ \\
\hline & & & 160.0 & 1.336 & 0.907 & 0.0033 & 0.0064 & 0.0072 & 0 . & 0.860 & 1.022 & & & & & \\
\hline \multirow[t]{2}{*}{1342223985} & 789 & 5575 & 100.0 & 1.631 & 1.469 & 0.0015 & 0.0059 & 0.0061 & 1.471 & 1.422 & 1.034 & 84 & 2 & 37 & 29.47 & $+0.97(\mathrm{TH})$ \\
\hline & & & 160.0 & 1.285 & 0.868 & 0.0032 & 0.0152 & 0.0156 & & 0.859 & 0.979 & & & & & \\
\hline \multirow[t]{2}{*}{1342223986} & 789 & 557 & 100.0 & 1.623 & 1.455 & 0.0014 & 0.0051 & 0.0053 & 1. & 1.422 & 1.024 & 20 & 2 & & 45 & + \\
\hline & & & 160.0 & 1.2 & 0.883 & 0.0033 & 0.0086 & 0.0092 & & 59 & 0.996 & & & & & \\
\hline \multirow[t]{2}{*}{1342235629} & 957 & 5592 & 70.0 & 1.9 & 1.630 & 022 & 83 & & 1.656 & 335 & 1.013 & 20.079 & 20.121 & L 2.828 & 14.56 & -0.4 \\
\hline & & & 160.0 & 1.326 & 0.868 & 0.0243 & 0.0151 & 0.0286 & 0.841 & 0.829 & 1.015 & & & & & \\
\hline \multirow[t]{2}{*}{1342235630} & 957 & 55921. & 70.0 & 1.978 & 1.684 & 0.0022 & 0.0207 & 0.0209 & 1.711 & 1.635 & 1.047 & 20.079 & 20.121 & L 2.828 & 14.63 & -0.4 \\
\hline & & & 160.0 & 1.276 & 0.838 & 0.0234 & 0.0449 & 0.0507 & 0 & 0.829 & 0.980 & & & & & \\
\hline \multirow[t]{2}{*}{1342235632} & 957 & 5592 & 100.0 & 1.666 & 1.449 & 0.0057 & 0.0058 & 0.0082 & 1.451 & 1.371 & 1.058 & 20.079 & 20.121 & L 2.828 & 14.74 & 3H) \\
\hline & & & 160.0 & 1.269 & 0.832 & 0.0246 & 0.0155 & 0.0291 & 0.806 & 0.829 & 0.972 & & & & & \\
\hline \multirow[t]{2}{*}{1342235633} & 957 & 55 & 100.0 & 1.689 & 1.466 & 0.0067 & 0.0093 & 0.0114 & 1.468 & 1.371 & 1.070 & 20.079 & 21 & 28 & 4.81 & BH) \\
\hline & & & 160.0 & 1.340 & 0.883 & 0.0233 & 0.0113 & 0.0259 & 0.8 & 0.829 & 1.032 & & & & & \\
\hline \multirow[t]{2}{*}{1342246772} & 1121 & 56086 & 70.0 & 1.895 & 1.573 & & & & & & 0.994 & 20.071 & 20.407 & Т 2.744 & 14.34 & \\
\hline & & & 160.0 & 1.349 & 0.858 & 0.0134 & 075 & 0.0154 & & 813 & 1.023 & & & & & \\
\hline \multirow[t]{2}{*}{1342246773} & 1121 & 56086.18904 & 70.0 & 1.967 & 1.630 & 0.0014 & 0.0382 & 0.0382 & 1.657 & 1.608 & 1.030 & 20.071 & 20.407 & Т 2.744 & 14.28 & $+0.35(\mathrm{BH})$ \\
\hline & & & 160.0 & 1.276 & 0.815 & 0.0138 & 0.0293 & 0.0324 & 0.789 & 0.813 & 0.971 & & & & & \\
\hline \multirow[t]{2}{*}{1342246774} & 1121 & 56086 & 100.0 & 1.556 & 1.318 & 0.0031 & 0.0094 & 0.0099 & 1.319 & 1.346 & 0.980 & 20.071 & 20.407 & Т 2.744 & 14.23 & +0.3 \\
\hline & & & 160.0 & 1.327 & 0.847 & 0.0143 & 0.0262 & 0.0299 & 0.820 & 0.813 & 1.009 & & & & & \\
\hline \multirow[t]{2}{*}{1342246775} & 1121 & 56086.19712 & 100.0 & 1.590 & 1.343 & 0.0026 & 0.0072 & 0.0077 & 1.344 & 1.346 & 0.999 & 20.071 & 20.407 & Т 2.744 & 14.18 & $+0.35(\mathrm{BH})$ \\
\hline & & & 160.0 & 1.274 & 0.813 & 0.0139 & 0.0205 & 0.0248 & 0.788 & 0.813 & 0.969 & & & & & \\
\hline \multirow[t]{2}{*}{1342257193} & 1310 & 56275.07361 & 70.0 & 1.9 & 1.724 & 1 & 0 & 0.0175 & & 5 & 034 & 20.057 & & & 23.80 & \\
\hline & & & 160.0 & 1.379 & 0.931 & 0.0047 & 0.0161 & 0.0168 & 0.902 & 0.857 & 1.052 & & & & & \\
\hline \multirow[t]{2}{*}{1342257194} & 1310 & 56275.07765 & 70.0 & 1.977 & 1.729 & 0.0012 & 0.0137 & 0.0138 & 1.757 & 1.695 & 1.037 & 20.057 & 19.821 & L 2.774 & 23.85 & $+0.76(\mathrm{TH})$ \\
\hline & & & 160.0 & 1.374 & 0.933 & 0.0048 & 0.0157 & 0.0165 & 0.904 & 0.857 & 1.055 & & & & & \\
\hline \multirow[t]{2}{*}{1342257195} & 1310 & 56275.08169 & 100.0 & 1.654 & 1.487 & 0.0014 & 0.0144 & 0.0145 & 1.488 & 1.419 & 1.048 & 20.057 & 19.821 & L 2.774 & 23.90 & $+0.76(\mathrm{TH})$ \\
\hline & & & 160.0 & 1.412 & 0.955 & 0.0047 & 0.0243 & 0.0248 & 0.925 & 0.857 & 1.079 & & & & & \\
\hline \multirow[t]{2}{*}{1342257196} & 1310 & 56275.08573 & 100.0 & 1.642 & 1.470 & 0.0017 & 0.0046 & 0.0049 & 1.471 & 1.419 & 1.037 & 20.057 & 19.821 & L 2.774 & 23.95 & $+0.76(\mathrm{TH})$ \\
\hline & & & 160.0 & 1.404 & 0.952 & 0.0047 & 0.0265 & 0.0269 & 0.923 & 0.857 & 1.077 & & & & & \\
\hline
\end{tabular}

Notes. Applied colour correction factors cc to derive colour corrected fluxes $f_{\text {moon,cc }}$, are listed in Table 7. $r_{\text {helio }}$ is the light-time corrected heliocentric range, $\Delta_{\mathrm{obs}}$ is the range of target centre wrt. the observer, i.e. Herschel, $\alpha$ is the phase angle with indication of $\mathrm{L}(\mathrm{eading})$ or $\mathrm{T}(\mathrm{railing})$ the Sun and $\theta_{\mathrm{U}-\mathrm{O}}$ is the angular separation from Uranus. $\dot{r}_{\text {helio }}$ is the heliocentric range rate and $\frac{\dot{r}_{\text {helio }}}{\left|\dot{r}_{\text {heli }}\right|} \mid$ indicates $\mathrm{L}$ (eading) $\mathrm{H}(\mathrm{emisphere})$ or T(railing) $\mathrm{H}$ (emisphere), if the absolute value of the ratio is greater than $\frac{2}{3}$, or $\mathrm{B}$ (oth) $\mathrm{H}$ (emispheres) otherwise. All five figures have been computed with the JPL Horizons On-Line Ephemeris System. The five epochs with 4 observations each are separated by horizontal lines. 
Table A.3. PSF photometry of Oberon.

\begin{tabular}{|c|c|c|c|c|c|c|c|c|c|c|c|c|c|c|c|c|}
\hline OBSID & OD & $\begin{array}{c}\text { MJD } \\
\text { mid-time obs. }\end{array}$ & $\begin{array}{l}\lambda_{\mathrm{ref}} \\
\mu \mathrm{m}\end{array}$ & $\begin{array}{l}\frac{f_{\text {moon }}}{f_{\text {Uranus }}} \\
\left(10^{-3}\right)\end{array}$ & $\begin{array}{c}f_{\text {moon }} \\
(\mathrm{Jy})\end{array}$ & $\begin{array}{l}\sigma_{\text {par }} \\
(\mathrm{Jy})\end{array}$ & $\begin{array}{l}\sigma_{\text {red }} \\
(\mathrm{Jy})\end{array}$ & $\begin{array}{l}\sigma_{\text {tot }} \\
(\mathrm{Jy})\end{array}$ & $\begin{array}{c}f_{\text {moon,cc }} \\
(\mathrm{Jy})\end{array}$ & $\begin{array}{c}f_{\text {model }} \\
(\mathrm{Jy})\end{array}$ & $\frac{f_{\text {moon, }, \mathrm{cc}}}{f_{\text {model }}}$ & $\begin{array}{l}r_{\text {helio }} \\
\text { (AU) }\end{array}$ & $\begin{array}{l}\Delta_{\mathrm{obs}} \\
(\mathrm{AU})\end{array}$ & $\begin{array}{c}\alpha \\
(\mathrm{deg})\end{array}$ & $\begin{array}{c}\theta_{\mathrm{U}-\mathrm{O}} \\
\left({ }^{\prime \prime}\right) \\
\end{array}$ & $\frac{\frac{h}{h \text { enlio }}}{\left|h_{\text {helio }}^{\text {max }}\right|}$ \\
\hline \multirow[t]{2}{*}{1342211117} & 579 & 55543.72060 & 70.0 & 1.892 & 1.639 & 0.0229 & 0.1034 & 0.1059 & 1.666 & 1.529 & 1.090 & 20.086 & 19.971 & L 2.826 & 6.31 & $+0.03(\mathrm{BH})$ \\
\hline & & & 160.0 & 1.212 & 0.806 & 0.1168 & 0.0594 & 0.1310 & 0.781 & 0.782 & 0.999 & & & & & \\
\hline \multirow[t]{2}{*}{1342211118} & 579 & 55543.72464 & 70.0 & 1.796 & 1.553 & 0.0241 & 0.0561 & 0.0610 & 1.578 & 1.529 & 1.032 & 20.086 & 19.971 & L 2.826 & 6.32 & $+0.03(\mathrm{BH})$ \\
\hline & & & 160.0 & 1.266 & 0.846 & 0.1205 & 0.0469 & 0.1293 & 0.819 & 0.782 & 1.048 & & & & & \\
\hline \multirow[t]{2}{*}{1342211120} & 579 & 55543.73128 & 100.0 & 1.612 & 1.425 & 0.0316 & 0.1059 & 0.1105 & 1.426 & 1.288 & 1.107 & 20.086 & 19.971 & L 2.826 & 6.35 & $+0.03(\mathrm{BH})$ \\
\hline & & & 160.0 & 1.226 & 0.817 & 0.1143 & 0.0902 & 0.1456 & 0.792 & 0.782 & 1.013 & & & & & \\
\hline \multirow[t]{2}{*}{1342211121} & 579 & 55543.73532 & 100.0 & 1.776 & 1.568 & 0.0319 & 0.0324 & 0.0454 & 1.569 & 1.288 & 1.218 & 20.086 & 19.971 & L 2.826 & 6.37 & $+0.03(\mathrm{BH})$ \\
\hline & & & 160.0 & 1.216 & 0.813 & 0.1161 & 0.0586 & 0.1300 & 0.788 & 0.782 & 1.007 & & & & & \\
\hline \multirow[t]{2}{*}{1342223982} & 789 & 55754.05794 & 70.0 & 1.828 & 1.611 & 0.0010 & 0.0077 & 0.0078 & 1.637 & 1.578 & 1.038 & 20.086 & 19.804 & Т 2.837 & 30.90 & -0.74 \\
\hline & & & 160.0 & 1.193 & 0.807 & 0.0029 & 0.0069 & 0.0075 & 0.781 & 0.803 & 0.973 & & & & & \\
\hline \multirow[t]{2}{*}{1342223983} & 789 & 55754.06198 & 70.0 & 1.867 & 1.638 & 0.0011 & 0.0116 & 0.0117 & 1.664 & 1.578 & 1.055 & 20.086 & 19.804 & Т 2.837 & 30.95 & $-0.74(\mathrm{LH})$ \\
\hline & & & 160.0 & 1.232 & 0.836 & 0.0028 & 0.0113 & 0.0116 & 0.810 & 0.803 & 1.009 & & & & & \\
\hline \multirow[t]{2}{*}{1342223985} & 789 & 55754.06862 & 100.0 & 1.526 & 1.374 & 0.0012 & 0.0114 & 0.0115 & 1.376 & 1.326 & 1.037 & 20.086 & 19.804 & Т 2.837 & 31.02 & $-0.74(\mathrm{LH})$ \\
\hline & & & 160.0 & 1.223 & 0.826 & 0.0029 & 0.0044 & 0.0052 & 0.801 & 0.803 & 0.997 & & & & & \\
\hline \multirow[t]{2}{*}{1342223986} & 789 & 55754.07266 & 100.0 & 1.559 & 1.398 & 0.0015 & 0.0127 & 0.0128 & 1.400 & 1.326 & 1.055 & 20.086 & 19.804 & Т 2.837 & 31.07 & $-0.74(\mathrm{LH})$ \\
\hline & & & 160.0 & 1.232 & 0.839 & 0.0028 & 0.0076 & 0.0081 & 0.813 & 0.803 & 1.012 & & & & & \\
\hline \multirow[t]{2}{*}{1342235629} & 957 & 55921.94924 & 70.0 & 1.842 & 1.573 & 0.0011 & 0.0138 & 0.0138 & 1.599 & 1.519 & 1.053 & 20.073 & 20.115 & L 2.829 & 24.69 & $+0.58(\mathrm{BH})$ \\
\hline & & & 160.0 & 1.251 & 0.819 & 0.0038 & 0.0106 & 0.0113 & 0.794 & 0.774 & 1.025 & & & & & \\
\hline \multirow[t]{2}{*}{1342235630} & 957 & 55921.95328 & 70.0 & 1.851 & 1.576 & 0.0012 & 0.0154 & 0.0154 & 1.601 & 1.519 & 1.054 & 20.073 & 20.115 & L 2.829 & 24.75 & $+0.58(\mathrm{BH})$ \\
\hline & & & 160.0 & 1.219 & 0.800 & 0.0038 & 0.0043 & 0.0058 & 0.776 & 0.774 & 1.002 & & & & & \\
\hline \multirow[t]{2}{*}{1342235632} & 957 & 55921.95992 & 100.0 & 1.517 & 1.320 & 0.0014 & 0.0089 & 0.0090 & 1.321 & 1.277 & 1.035 & 20.073 & 20.115 & L 2.829 & 24.84 & $+0.59(\mathrm{BH})$ \\
\hline & & & 160.0 & 1.241 & 0.814 & 0.0037 & 0.0082 & 0.0090 & 0.789 & 0.774 & 1.019 & & & & & \\
\hline \multirow[t]{2}{*}{1342235633} & 957 & 55921.96396 & 100.0 & 1.558 & 1.353 & 0.0019 & 0.0086 & 0.0088 & 1.354 & 1.277 & 1.060 & 20.073 & 20.115 & L 2.829 & 24.89 & $+0.59(\mathrm{BH})$ \\
\hline & & & 160.0 & 1.244 & 0.819 & 0.0038 & 0.0125 & 0.0130 & 0.794 & 0.774 & 1.026 & & & & & \\
\hline \multirow[t]{2}{*}{1342246772} & 1121 & 56086.18500 & 70.0 & 1.923 & 1.597 & 0.0012 & 0.0334 & 0.0335 & 1.623 & 1.500 & 1.082 & 20.070 & 20.406 & Т 2.744 & 37.58 & $+0.95(\mathrm{TH})$ \\
\hline & & & 160.0 & 1.246 & 0.792 & 0.0031 & 0.0089 & 0.0095 & 0.768 & 0.761 & 1.009 & & & & & \\
\hline \multirow[t]{2}{*}{1342246773} & 1121 & 56086.18904 & 70.0 & 1.890 & 1.566 & 0.0012 & 0.0328 & 0.0328 & 1.591 & 1.500 & 1.061 & 20.070 & 20.406 & Т 2.744 & 37.56 & $+0.95(\mathrm{TH})$ \\
\hline & & & 160.0 & 1.215 & 0.776 & 0.0034 & 0.0081 & 0.0088 & 0.752 & 0.761 & 0.988 & & & & & \\
\hline \multirow[t]{2}{*}{1342246774} & 1121 & 56086.19308 & 100.0 & 1.535 & 1.300 & 0.0014 & 0.0057 & 0.0059 & 1.301 & 1.258 & 1.034 & 20.070 & 20.406 & Т 2.744 & 37.54 & $+0.95(\mathrm{TH})$ \\
\hline & & & 160.0 & 1.225 & 0.781 & 0.0031 & 0.0043 & 0.0053 & 0.757 & 0.761 & 0.995 & & & & & \\
\hline \multirow[t]{2}{*}{1342246775} & 1121 & 56086.19712 & 100.0 & 1.525 & 1.289 & 0.0015 & 0.0074 & 0.0075 & 1.290 & 1.258 & 1.025 & 20.070 & 20.405 & Т 2.744 & 37.52 & $+0.95(\mathrm{TH})$ \\
\hline & & & 160.0 & 1.195 & 0.762 & 0.0034 & 0.0070 & 0.0077 & 0.739 & 0.761 & 0.971 & & & & & \\
\hline \multirow[t]{2}{*}{1342257193} & 1310 & 56275.07361 & 70.0 & 1.850 & 1.625 & 0.0011 & 0.0129 & 0.0129 & 1.651 & 1.579 & 1.046 & 20.060 & 19.824 & L 2.773 & 35.88 & $+0.86(\mathrm{TH})$ \\
\hline & & & 160.0 & 1.201 & 0.811 & 0.0032 & 0.0054 & 0.0063 & 0.785 & 0.802 & 0.979 & & & & & \\
\hline \multirow[t]{2}{*}{1342257194} & 1310 & 56275.07765 & 70.0 & 1.866 & 1.632 & 0.0011 & 0.0176 & 0.0177 & 1.659 & 1.579 & 1.051 & 20.060 & 19.824 & L 2.773 & 35.84 & $+0.86(\mathrm{TH})$ \\
\hline & & & 160.0 & 1.202 & 0.816 & 0.0033 & 0.0036 & 0.0049 & 0.791 & 0.802 & 0.986 & & & & & \\
\hline \multirow[t]{2}{*}{1342257195} & 1310 & 56275.08169 & 100.0 & 1.553 & 1.396 & 0.0013 & 0.0148 & 0.0149 & 1.397 & 1.325 & 1.055 & 20.060 & 19.824 & L 2.773 & 35.81 & $+0.86(\mathrm{TH})$ \\
\hline & & & 160.0 & 1.224 & 0.828 & 0.0031 & 0.0044 & 0.0054 & 0.802 & 0.802 & 1.000 & & & & & \\
\hline \multirow[t]{2}{*}{1342257196} & 1310 & 56275.08573 & 100.0 & 1.544 & 1.382 & 0.0013 & 0.0128 & 0.0128 & 1.383 & 1.325 & 1.044 & 20.061 & 19.825 & L 2.773 & 35.78 & $+0.86(\mathrm{TH})$ \\
\hline & & & 160.0 & 1.178 & 0.799 & 0.0033 & 0.0097 & 0.0103 & 0.775 & 0.802 & 0.966 & & & & & \\
\hline
\end{tabular}

Notes. Applied colour correction factors cc to derive colour corrected fluxes $\mathrm{f}_{\text {moon,cc }}$, are listed in Table 7. $\mathrm{r}_{\text {helio }}$ is the light-time corrected heliocentric range, $\Delta_{\mathrm{obs}}$ is the range of target centre wrt. the observer, i.e. Herschel, $\alpha$ is the Phase angle with indication of $\mathrm{L}$ (eading) or T(railing) the Sun, and $\theta_{\mathrm{U}-\mathrm{O}}$ is the angular separation from Uranus. $\dot{r}_{\text {helio }}$ is the heliocentric range rate and $\frac{\dot{i}_{\text {helio }}}{\left|\dot{r}_{\text {heli }}^{\text {mal }}\right|}$ indicates $\mathrm{L}$ (eading) $\mathrm{H}$ (emisphere) or T(railing) $\mathrm{H}$ (emisphere), if the absolute value of the ratio is greater than $\frac{2}{3}$, or $\mathrm{B}$ (oth) $\mathrm{H}$ (emispheres) otherwise. All five figures have been computed with the JPL Horizons On-Line Ephemeris System. The five epochs with 4 observations each are separated by horizontal lines. 
Table A.4. PSF photometry of Umbriel.

\begin{tabular}{|c|c|c|c|c|c|c|c|c|c|c|c|c|c|c|c|c|}
\hline OBSID & OD & $\begin{array}{c}\text { MJD } \\
\text { mid-time obs. }\end{array}$ & $\begin{array}{l}\lambda_{\text {ref }} \\
\mu \mathrm{m}\end{array}$ & $\begin{array}{c}\frac{f_{\text {moon }}}{f \text { Uranus }} \\
\left(10^{-3}\right)\end{array}$ & $\begin{array}{c}f_{\text {moon }} \\
(\mathrm{Jy})\end{array}$ & $\begin{array}{l}\sigma_{\mathrm{par}} \\
(\mathrm{Jy})\end{array}$ & $\begin{array}{l}\sigma_{\text {red }} \\
(\mathrm{Jy})\end{array}$ & $\begin{array}{l}\sigma_{\text {tot }} \\
(\mathrm{Jy})\end{array}$ & $\begin{array}{c}f_{\text {moon,cc }} \\
(\mathrm{Jy})\end{array}$ & $\begin{array}{c}f_{\text {model }} \\
(\mathrm{Jy})\end{array}$ & $\frac{f_{\text {moon,cc }}}{f_{\text {model }}}$ & $\begin{array}{l}r_{\text {helio }} \\
(\mathrm{AU})\end{array}$ & $\begin{array}{l}\Delta_{\mathrm{obs}} \\
(\mathrm{AU})\end{array}$ & $\begin{array}{c}\alpha \\
(\mathrm{deg})\end{array}$ & $\begin{array}{c}\theta_{\mathrm{U}-\mathrm{O}} \\
\left({ }^{\prime \prime}\right)\end{array}$ & $\frac{\dot{r}_{\text {helio }}}{\left|\dot{r}_{\text {hellio }}^{\text {max }}\right|}$ \\
\hline 17 & 5 & 543.72060 & 70.0 & 0.962 & 0.833 & 0.0035 & 0.0182 & 0.0185 & 0.847 & 0.915 & 0.926 & 20.091 & .976 & 25 & 16.59 & -0.90 \\
\hline 1342211118 & 579 & 55543.72464 & $\begin{array}{r}10.0 \\
70.0 \\
160.0\end{array}$ & $\begin{array}{l}0.062 \\
1.017 \\
0.666\end{array}$ & $\begin{array}{l}0.440 \\
0.880 \\
0.445\end{array}$ & $\begin{array}{l}0.0380 \\
0.0034 \\
0.0374\end{array}$ & $\begin{array}{l}0.0313 \\
0.0116 \\
0.0304\end{array}$ & $\begin{array}{l}0.0494 \\
0.0121 \\
0.0482\end{array}$ & $\begin{array}{l}0.427 \\
0.894 \\
0.431\end{array}$ & $\begin{array}{l}0.463 \\
0.915 \\
0.463\end{array}$ & $\begin{array}{l}0.921 \\
0.977 \\
0.931\end{array}$ & 20.091 & 9.976 & L 2.825 & 16.64 & $-0.90(\mathrm{LH})$ \\
\hline 342211120 & 579 & 55543.73128 & $\begin{array}{l}100.0 \\
160.0\end{array}$ & $\begin{array}{l}0.812 \\
0.657\end{array}$ & $\begin{array}{l}0.718 \\
0.438\end{array}$ & $\begin{array}{l}0.0059 \\
0.0380\end{array}$ & $\begin{array}{l}0.0076 \\
0.0235\end{array}$ & $\begin{array}{l}0.0096 \\
0.0446\end{array}$ & $\begin{array}{l}0.719 \\
0.425\end{array}$ & $\begin{array}{l}0.766 \\
0.463\end{array}$ & $\begin{array}{l}0.938 \\
0.917\end{array}$ & 20.091 & 19.976 & L 2.825 & 16.71 & $-0.91(\mathrm{LH})$ \\
\hline 1342211121 & 579 & 55543.73532 & $\begin{array}{l}100.0 \\
160.0\end{array}$ & $\begin{array}{l}0.863 \\
0.700\end{array}$ & $\begin{array}{l}0.762 \\
0.468\end{array}$ & $\begin{array}{l}0.0059 \\
0.0378\end{array}$ & $\begin{array}{l}0.0051 \\
0.0311\end{array}$ & $\begin{array}{l}0.0078 \\
0.0489\end{array}$ & $\begin{array}{l}0.763 \\
0.453\end{array}$ & $\begin{array}{l}0.766 \\
0.463\end{array}$ & $\begin{array}{l}0.996 \\
0.979\end{array}$ & 20.091 & 19.976 & L 2.826 & 6.76 & $-001 / \mathrm{LH}$ \\
\hline 13422 & 789 & 55754.057 & $\begin{array}{r}70.0 \\
160.0\end{array}$ & $\begin{array}{l}1.043 \\
0.707\end{array}$ & $\begin{array}{l}0.919 \\
0.478\end{array}$ & $\begin{array}{l}0.0107 \\
0.0942\end{array}$ & $\begin{array}{l}0.0157 \\
0.0687\end{array}$ & $\begin{array}{l}0.0189 \\
0.1166\end{array}$ & & $\begin{array}{l}0.941 \\
0.474\end{array}$ & $\begin{array}{l}0.992 \\
0.977\end{array}$ & 20.085 & 19.803 & Т 2.837 & 8.62 & $\mathrm{BH})$ \\
\hline 1342223983 & 789 & 55754.06198 & $\begin{array}{r}70.0 \\
160.0\end{array}$ & $\begin{array}{l}1.062 \\
0.638\end{array}$ & $\begin{array}{l}0.932 \\
0.433\end{array}$ & $\begin{array}{l}0.0080 \\
0.0911\end{array}$ & $\begin{array}{l}0.0200 \\
0.0345\end{array}$ & $\begin{array}{l}0.0215 \\
0.0974\end{array}$ & & & & 20.085 & 19.803 & Т 2.837 & 8.54 & $+0.37(\mathrm{BH})$ \\
\hline 885 & 789 & 5575 & $\begin{array}{l}100.0 \\
160.0\end{array}$ & & & $\begin{array}{l}0.0235 \\
0.1010\end{array}$ & $\begin{array}{l}0.0144 \\
0.0956\end{array}$ & $\begin{array}{l}0.0275 \\
0.1391\end{array}$ & & & & 20.085 & .803 & Т 2.836 & .42 & $+0.36(\mathrm{BH})$ \\
\hline 13 & 789 & 557 & $\begin{array}{l}100.0 \\
160.0\end{array}$ & $\begin{array}{l}0.884 \\
0.715\end{array}$ & $\begin{array}{l}0.793 \\
0.487\end{array}$ & $\begin{array}{l}0.0196 \\
0.0954\end{array}$ & $\begin{array}{l}0.0125 \\
0.0522\end{array}$ & $\begin{array}{l}0.0232 \\
0.1088\end{array}$ & & & & 85 & 33 & Т 2.836 & 34 & $-0.36(\mathrm{BH})$ \\
\hline 13 & 957 & 24 & $\begin{array}{r}70.0 \\
160.0\end{array}$ & & $\begin{array}{l}0.773 \\
0.417\end{array}$ & & $\begin{array}{l}0.0502 \\
0.0313\end{array}$ & & & & $\begin{array}{l}0.866 \\
0.884\end{array}$ & 20.075 & 20.116 & 2.828 & 7.09 & (BH) \\
\hline 13 & 957 & 559 & $\begin{array}{r}70.0 \\
160.0\end{array}$ & $\begin{array}{l}0.991 \\
0.646\end{array}$ & $\begin{array}{l}0.843 \\
0.425\end{array}$ & $\begin{array}{l}0.0199 \\
0.1646\end{array}$ & $\begin{array}{l}0.0250 \\
0.1205\end{array}$ & $\begin{array}{l}0.0320 \\
0.2040\end{array}$ & & & & 20.075 & 0.116 & L 2.828 & .01 & $-0.33(\mathrm{BH})$ \\
\hline 1342235632 & 05. & 5 & $\begin{array}{l}100.0 \\
160.0\end{array}$ & $\begin{array}{l}0.747 \\
0.742\end{array}$ & $\begin{array}{l}0.650 \\
0.486\end{array}$ & $\begin{array}{l}0.0463 \\
0.1785\end{array}$ & $\begin{array}{l}0.0437 \\
0.0488\end{array}$ & $\begin{array}{l}0.0636 \\
0.1850\end{array}$ & & & & 75 & 20.117 & L 2.828 & 60 & $-0.32(\mathrm{BH})$ \\
\hline & 957 & & $\begin{array}{l}100.0 \\
160.0\end{array}$ & $\begin{array}{l}0.815 \\
0.584\end{array}$ & $\begin{array}{l}0.708 \\
0.385\end{array}$ & $\begin{array}{l}0.0425 \\
0.1688\end{array}$ & $\begin{array}{l}0.0293 \\
0.0519\end{array}$ & & & & & 75 & 17 & & 8 & (BH) \\
\hline 72 & 1121 & 0 & $\begin{array}{r}70.0 \\
160.0\end{array}$ & $\begin{array}{l}1.022 \\
0.616\end{array}$ & $\begin{array}{l}0.848 \\
0.392\end{array}$ & $\begin{array}{l}0.0063 \\
0.0724\end{array}$ & $\begin{array}{l}0.0173 \\
0.0611\end{array}$ & $\begin{array}{l}0.0184 \\
0.0947\end{array}$ & & $\begin{array}{l}0.892 \\
0.448\end{array}$ & $\begin{array}{l}0.966 \\
0.847\end{array}$ & 20.070 & 0.406 & 2.744 & 10.30 & $\overline{\mathrm{BH})}$ \\
\hline 134 & 1121 & 5608 & $\begin{array}{r}70.0 \\
160.0\end{array}$ & $\begin{array}{l}1.072 \\
0.646\end{array}$ & $\begin{array}{l}0.889 \\
0.412\end{array}$ & $\begin{array}{l}0.0082 \\
0.0787\end{array}$ & $\begin{array}{l}0.0275 \\
0.0508\end{array}$ & $\begin{array}{l}0.0287 \\
0.0936\end{array}$ & $\begin{array}{l}0.903 \\
0.399\end{array}$ & $\begin{array}{l}0.892 \\
0.448\end{array}$ & $\begin{array}{l}1.013 \\
0.892\end{array}$ & 20.070 & .406 & Г 2.744 & 10.37 & $-0.49(\mathrm{BH})$ \\
\hline 13 & 1121 & 5 & $\begin{array}{l}100.0 \\
160.0\end{array}$ & $\begin{array}{l}0.778 \\
0.570\end{array}$ & $\begin{array}{l}0.659 \\
0.363\end{array}$ & $\begin{array}{l}0.0132 \\
0.0773\end{array}$ & $\begin{array}{l}0.0086 \\
0.0651\end{array}$ & $\begin{array}{l}0.0157 \\
0.1011\end{array}$ & $\begin{array}{l}0.660 \\
0.352\end{array}$ & $\begin{array}{l}0.744 \\
0.448\end{array}$ & $\begin{array}{l}0.887 \\
0.786\end{array}$ & 70 & 06 & 44 & .44 & H) \\
\hline 13 & 1121 & 56 & $\begin{array}{l}100.0 \\
160.0\end{array}$ & $\begin{array}{l}0.736 \\
0.442\end{array}$ & $\begin{array}{l}0.622 \\
0.282\end{array}$ & $\begin{array}{l}0.0162 \\
0.0734\end{array}$ & $\begin{array}{l}0.0308 \\
0.0477\end{array}$ & $\begin{array}{l}0.0348 \\
0.0876\end{array}$ & $\begin{array}{l}0.623 \\
0.273\end{array}$ & $\begin{array}{l}0.744 \\
0.448\end{array}$ & $\begin{array}{l}0.837 \\
0.610\end{array}$ & 20.070 & 20.406 & Т 2.744 & 10.51 & H) \\
\hline 1342257193 & 1310 & 56275.07361 & $\begin{array}{r}70.0 \\
160.0\end{array}$ & $\begin{array}{l}1.078 \\
0.510\end{array}$ & $\begin{array}{l}0.947 \\
0.344\end{array}$ & $\begin{array}{l}0.0029 \\
0.0344\end{array}$ & $\begin{array}{l}.0075 \\
.0396\end{array}$ & $\begin{array}{l}0.0081 \\
0.0524\end{array}$ & 0.333 & $\begin{array}{l}0.940 \\
0.473\end{array}$ & $\begin{array}{l}1.024 \\
0.705\end{array}$ & 58 & 22 & 774 & .83 & H) \\
\hline 94 & 1310 & 56275.07765 & $\begin{array}{r}70.0 \\
160.0\end{array}$ & $\begin{array}{l}1.102 \\
0.573\end{array}$ & $\begin{array}{l}0.964 \\
0.389\end{array}$ & $\begin{array}{l}0.0034 \\
0.0314\end{array}$ & $\begin{array}{l}0.0104 \\
0.0435\end{array}$ & $\begin{array}{l}0.0110 \\
0.0536\end{array}$ & $\begin{array}{l}0.980 \\
0.377\end{array}$ & $\begin{array}{l}0.940 \\
0.473\end{array}$ & $\begin{array}{l}1.042 \\
0.797\end{array}$ & 20.058 & 9.822 & 2.774 & 15.88 & $+0.85(\mathrm{TH})$ \\
\hline 1342257195 & 1310 & 56275.08169 & $\begin{array}{l}100.0 \\
160.0\end{array}$ & $\begin{array}{l}0.905 \\
0.523\end{array}$ & $\begin{array}{l}0.814 \\
0.354\end{array}$ & $\begin{array}{l}0.0064 \\
0.0373\end{array}$ & $\begin{array}{l}.0042 \\
.0469\end{array}$ & $\begin{array}{l}0.0077 \\
0.0599\end{array}$ & $\begin{array}{l}0.815 \\
0.343\end{array}$ & $\begin{array}{l}0.785 \\
0.473\end{array}$ & $\begin{array}{l}1.038 \\
0.725\end{array}$ & 20.058 & 19.822 & 2.774 & 15.94 & $+0.85(\mathrm{TH})$ \\
\hline 1342257196 & 1310 & 56275.08573 & $\begin{array}{l}100.0 \\
160.0\end{array}$ & $\begin{array}{l}0.896 \\
0.445\end{array}$ & $\begin{array}{l}0.802 \\
0.302\end{array}$ & $\begin{array}{l}0.0056 \\
0.0380\end{array}$ & $\begin{array}{l}0.0038 \\
0.0625\end{array}$ & $\begin{array}{l}0.0068 \\
0.0731\end{array}$ & $\begin{array}{l}0.803 \\
0.293\end{array}$ & $\begin{array}{l}0.785 \\
0.473\end{array}$ & $\begin{array}{l}1.023 \\
0.618\end{array}$ & 20.058 & 19.822 & L 2.774 & 15.99 & $+0.86(\mathrm{TH})$ \\
\hline
\end{tabular}

Notes. Applied colour correction factors cc to derive colour corrected fluxes $f_{\text {moon,cc }}$, are listed in Table 7. $r_{\text {helio }}$ is the light-time corrected heliocentric range, $\Delta_{\mathrm{obs}}$ is the range of target centre wrt. the observer, i.e. Herschel, $\alpha$ is the Phase angle with indication of $\mathrm{L}(\mathrm{eading})$ or $\mathrm{T}(\mathrm{railing})$ the Sun, and $\theta_{\mathrm{U}-\mathrm{O}}$ is the angular separation from Uranus. $\dot{r}_{\text {helio }}$ is the heliocentric range rate and $\frac{\dot{r}_{\text {helio }}}{\left|\dot{r}_{\text {helio }}\right|}$ indicates $\mathrm{L}$ (eading) $\mathrm{H}(\mathrm{emisphere})$ or T(railing) $\mathrm{H}$ (emisphere), if the absolute value of the ratio is greater than $\frac{2}{3}$, or $\mathrm{B}(\mathrm{oth}) \mathrm{H}$ (emispheres) otherwise. All five figures have been computed with the JPL Horizons On-Line Ephemeris System. The five epochs with 4 observations each are separated by horizontal lines. 
Table A.5. PSF photometry of Ariel.

\begin{tabular}{|c|c|c|c|c|c|c|c|c|c|c|c|c|c|c|c|c|}
\hline OBSID & OD & $\begin{array}{c}\text { MJD } \\
\text { mid-time obs. }\end{array}$ & $\begin{array}{l}\lambda_{\mathrm{ref}} \\
\mu \mathrm{m}\end{array}$ & $\begin{array}{l}\frac{f_{\text {moon }}}{f f_{\text {Uranus }}} \\
\left(10^{-3}\right)\end{array}$ & $\begin{array}{c}f_{\text {moon }} \\
(\mathrm{Jy})\end{array}$ & $\begin{array}{l}\sigma_{\mathrm{par}} \\
(\mathrm{Jy})\end{array}$ & $\begin{array}{c}\sigma_{\text {red }} \\
(\mathrm{Jy})\end{array}$ & $\begin{array}{l}\sigma_{\text {tot }} \\
(\mathrm{Jy})\end{array}$ & $\begin{array}{c}f_{\text {moon,cc }} \\
(\mathrm{Jy})\end{array}$ & $\begin{array}{c}f_{\text {model }} \\
(\mathrm{Jy})\end{array}$ & $\frac{f_{\text {moon,cc }}}{f_{\text {model }}}$ & $\begin{array}{l}r_{\text {helio }} \\
\text { (AU) }\end{array}$ & $\begin{array}{l}\Delta_{\mathrm{obs}} \\
(\mathrm{AU})\end{array}$ & $\begin{array}{c}\alpha \\
(\operatorname{deg})\end{array}$ & $\begin{array}{c}\theta_{\mathrm{U}-\mathrm{O}} \\
\left(^{\prime \prime}\right)\end{array}$ & $\frac{\dot{h}_{\text {helio }}}{\left|\dot{r i m}_{\text {helio }}^{\text {max }}\right|}$ \\
\hline 422 & 9 & 55543.72060 & $\begin{array}{r}70.0 \\
160.0\end{array}$ & $\begin{array}{l}0.859 \\
0.278\end{array}$ & $\begin{array}{l}0.745 \\
0.185\end{array}$ & $\begin{array}{l}0.0063 \\
0.1387\end{array}$ & $\begin{array}{l}0.0093 \\
0.0797\end{array}$ & $\begin{array}{l}0.0112 \\
0.1600\end{array}$ & $\begin{array}{l}0.758 \\
0.179\end{array}$ & $\begin{array}{l}0.751 \\
0.410\end{array}$ & $\begin{array}{l}1.009 \\
0.438\end{array}$ & 20.090 & 19.975 & 26 & 13.15 & $+1.00(\mathrm{TH})$ \\
\hline 342211118 & 579 & 55543.72464 & $\begin{array}{r}70.0 \\
160.0\end{array}$ & $\begin{array}{l}0.845 \\
0.364\end{array}$ & $\begin{array}{l}0.730 \\
0.243\end{array}$ & $\begin{array}{l}0.0069 \\
0.1345\end{array}$ & $\begin{array}{l}0.0236 \\
0.0906\end{array}$ & $\begin{array}{l}0.0246 \\
0.1621\end{array}$ & $\begin{array}{l}0.743 \\
0.236\end{array}$ & $\begin{array}{l}0.751 \\
0.410\end{array}$ & $\begin{array}{l}0.989 \\
0.576\end{array}$ & 20.090 & 19.975 & L 2.826 & 13.14 & $-100 / \mathrm{TH}$ \\
\hline 342211120 & 579 & 55543.73128 & $\begin{array}{l}100.0 \\
160.0\end{array}$ & $\begin{array}{l}0.728 \\
0.333\end{array}$ & $\begin{array}{l}0.644 \\
0.222\end{array}$ & $\begin{array}{l}0.0130 \\
0.1316\end{array}$ & $\begin{array}{l}0.0285 \\
0.0938\end{array}$ & $\begin{array}{l}0.0313 \\
0.1616\end{array}$ & $\begin{array}{l}0.645 \\
0.216\end{array}$ & $\begin{array}{l}0.656 \\
0.410\end{array}$ & $\begin{array}{l}0.984 \\
0.526\end{array}$ & 20.090 & 19.975 & L 2.826 & 13.12 & +100 (TH \\
\hline 42211121 & 579 & 55543.73532 & $\begin{array}{l}100.0 \\
160.0\end{array}$ & $\begin{array}{l}0.660 \\
0.413\end{array}$ & $\begin{array}{l}0.582 \\
0.276\end{array}$ & $\begin{array}{l}0.0148 \\
0.1188\end{array}$ & $\begin{array}{l}0.0201 \\
0.0816\end{array}$ & $\begin{array}{l}0.0249 \\
0.1442\end{array}$ & $\begin{array}{l}0.584 \\
0.268\end{array}$ & $\begin{array}{l}0.656 \\
0.410\end{array}$ & $\begin{array}{l}0.890 \\
0.654\end{array}$ & 20.090 & 19.975 & L 2.826 & 3.11 & (TL \\
\hline 13 & 789 & 5575 & $\begin{array}{r}70.0 \\
160.0\end{array}$ & $\begin{array}{l}0.932 \\
0.345\end{array}$ & $\begin{array}{l}0.821 \\
0.233\end{array}$ & $\begin{array}{l}0.0052 \\
0.0974\end{array}$ & $\begin{array}{l}0.0107 \\
0.0681\end{array}$ & $\begin{array}{l}0.0119 \\
0.1188\end{array}$ & & & & 20.083 & 9.802 & 2.837 & 3.05 & H) \\
\hline 13422 & 789 & 55754 & $\begin{array}{r}70.0 \\
160.0\end{array}$ & & & & & $\begin{array}{l}0.0232 \\
0.1286\end{array}$ & & & & 20.083 & 19.802 & Т 2.837 & 13.07 & $-0.98(\mathrm{LH})$ \\
\hline 13422 & 789 & 557 & $\begin{array}{l}100.0 \\
160.0\end{array}$ & & & & & & & & & 20.083 & .801 & 2.837 & 3.11 & -098(L H) \\
\hline 13 & 789 & 5 & $\begin{array}{l}100.0 \\
160.0\end{array}$ & $\begin{array}{l}0.943 \\
0.224\end{array}$ & $\begin{array}{l}0.846 \\
0.153\end{array}$ & $\begin{array}{l}0.0122 \\
0.0886\end{array}$ & $\begin{array}{l}0.0153 \\
0.0798\end{array}$ & $\begin{array}{l}0.0196 \\
0.1193\end{array}$ & $\begin{array}{l}0.848 \\
0.148\end{array}$ & $\begin{array}{l}0.678 \\
0.422\end{array}$ & $\begin{array}{l}1.251 \\
0.351\end{array}$ & 33 & 01 & 37 & .13 & $000 \Omega$ \\
\hline $13 \quad \mathrm{~s}$ & 957 & 559 & $\begin{array}{r}70.0 \\
160.0\end{array}$ & $\begin{array}{l}0.850 \\
0.250\end{array}$ & 0.726 & & & $\begin{array}{l}0.0343 \\
0.1434\end{array}$ & & & & 20.077 & 20.119 & 29 & 11.59 & TH) \\
\hline 13 & 957 & 5 & $\begin{array}{r}70.0 \\
160.0\end{array}$ & & & & & & & & & .077 & 19 & 2.829 & 53 & $-0.87(\mathrm{TH})$ \\
\hline 1342235632 & 9 & 5 & $\begin{array}{l}100.0 \\
160.0\end{array}$ & & & & & & & & & 7 & 19 & & 43 & TTI \\
\hline 33 & 957 & 55 & $\begin{array}{l}100.0 \\
160.0\end{array}$ & $\begin{array}{l}0.683 \\
0.232\end{array}$ & $\begin{array}{l}0.593 \\
0.153\end{array}$ & & $\begin{array}{l}0.0206 \\
0.0450\end{array}$ & $\begin{array}{l}0.0310 \\
0.1404\end{array}$ & & & & 7 & 19 & 28 & .37 & (TH) \\
\hline 1342246772 & 21 & 5 & $\begin{array}{r}70.0 \\
160.0\end{array}$ & $\begin{array}{l}0.852 \\
0.072\end{array}$ & $\begin{array}{l}0.708 \\
0.046\end{array}$ & $\begin{array}{l}0.0811 \\
1.6721\end{array}$ & $\begin{array}{l}0.1371 \\
0.0569\end{array}$ & $\begin{array}{l}0.1593 \\
1.6731\end{array}$ & & $\begin{array}{l}0.743 \\
0.401\end{array}$ & $\begin{array}{c}0.969 \\
0.111\end{array}$ & 70 & 06 & 44 & 47 & $\mathrm{BH})$ \\
\hline 13 & 1121 & 5 & $\begin{array}{r}70.0 \\
160.0\end{array}$ & $\begin{array}{l}0.633 \\
0.200\end{array}$ & $\begin{array}{l}0.525 \\
0.128\end{array}$ & $\begin{array}{l}0.0778 \\
0.6343\end{array}$ & $\begin{array}{l}0.1864 \\
0.1172\end{array}$ & $\begin{array}{l}0.2020 \\
0.6450\end{array}$ & & $\begin{array}{l}0.743 \\
0.401\end{array}$ & $\begin{array}{l}0.718 \\
0.309\end{array}$ & 0 & 6 & 44 & 46 & (BH) \\
\hline 13 & 1121 & 5 & $\begin{array}{l}100.0 \\
160.0\end{array}$ & $\begin{array}{l}1.077 \\
0.303\end{array}$ & $\begin{array}{l}0.912 \\
0.193\end{array}$ & $\begin{array}{l}0.1160 \\
0.6355\end{array}$ & $\begin{array}{l}0.0960 \\
0.0828\end{array}$ & $\begin{array}{l}0.1506 \\
0.6409\end{array}$ & $\begin{array}{l}0.915 \\
0.188\end{array}$ & $\begin{array}{l}0.645 \\
0.401\end{array}$ & $\begin{array}{l}1.418 \\
0.468\end{array}$ & 70 & 06 & 44 & 45 & H) \\
\hline 75 & 1121 & 5608 & $\begin{array}{l}100.0 \\
160.0\end{array}$ & $\begin{array}{l}1.215 \\
0.663\end{array}$ & $\begin{array}{l}1.027 \\
0.423\end{array}$ & $\begin{array}{l}0.1131 \\
0.7415\end{array}$ & $\begin{array}{l}0.0851 \\
0.3194\end{array}$ & $\begin{array}{l}0.1415 \\
0.8073\end{array}$ & $\begin{array}{l}1.030 \\
0.410\end{array}$ & $\begin{array}{l}0.645 \\
0.401\end{array}$ & $\begin{array}{l}1.597 \\
1.024\end{array}$ & 20.070 & 20.405 & 44 & 4.45 & (BH) \\
\hline 13 & 1310 & 5 & $\begin{array}{r}70.0 \\
160.0\end{array}$ & $\begin{array}{l}0.663 \\
0.499\end{array}$ & $\begin{array}{l}0.582 \\
0.337\end{array}$ & $\begin{array}{l}0.0510 \\
0.3590\end{array}$ & $\begin{array}{l}.1044 \\
.1382\end{array}$ & $\begin{array}{l}0.1162 \\
0.3847\end{array}$ & 0.327 & $\begin{array}{l}0.781 \\
0.422\end{array}$ & 0.775 & 60 & 24 & 774 & 13 & $3 \mathrm{H})$ \\
\hline 1342 & 1310 & 5627 & $\begin{array}{r}70.0 \\
160.0\end{array}$ & $\begin{array}{l}0.751 \\
0.246\end{array}$ & $\begin{array}{l}0.657 \\
0.167\end{array}$ & $\begin{array}{l}0.0442 \\
0.3202\end{array}$ & $\begin{array}{l}0.0321 \\
0.1194\end{array}$ & $\begin{array}{l}0.0546 \\
0.3418\end{array}$ & $\begin{array}{l}0.668 \\
0.162\end{array}$ & $\begin{array}{l}0.781 \\
0.422\end{array}$ & $\begin{array}{l}0.856 \\
0.384\end{array}$ & 20.060 & 19.824 & 2.774 & 6.03 & $5(\mathrm{BH})$ \\
\hline 1342257195 & 1310 & 56275.08169 & $\begin{array}{l}100.0 \\
160.0\end{array}$ & $\begin{array}{l}0.503 \\
0.214\end{array}$ & $\begin{array}{l}0.452 \\
0.144\end{array}$ & $\begin{array}{l}0.0673 \\
0.3606\end{array}$ & $\begin{array}{l}0.0493 \\
0.0476\end{array}$ & $\begin{array}{l}0.0834 \\
0.3637\end{array}$ & $\begin{array}{l}0.453 \\
0.140\end{array}$ & $\begin{array}{l}0.678 \\
0.422\end{array}$ & $\begin{array}{l}0.668 \\
0.332\end{array}$ & 20.060 & 19.824 & L 2.774 & 5.94 & $+0.34(\mathrm{BH})$ \\
\hline 13422 & 1310 & 56275.08573 & $\begin{array}{l}100.0 \\
160.0\end{array}$ & $\begin{array}{l}0.678 \\
0.473\end{array}$ & $\begin{array}{l}0.607 \\
0.321\end{array}$ & $\begin{array}{l}0.0604 \\
0.3281\end{array}$ & $\begin{array}{l}0.0215 \\
0.1268\end{array}$ & $\begin{array}{l}0.0641 \\
0.3517\end{array}$ & $\begin{array}{l}0.609 \\
0.312\end{array}$ & $\begin{array}{l}0.678 \\
0.422\end{array}$ & $\begin{array}{l}0.898 \\
0.739\end{array}$ & 20.060 & 19.824 & L 2.774 & 5.85 & $+0.33(\mathrm{BH})$ \\
\hline
\end{tabular}

Notes. Applied colour correction factors cc to derive colour corrected fluxes $f_{\text {moon,cc }}$, are listed in Table 7. $r_{\text {helio }}$ is the light-time corrected heliocentric range, $\Delta_{\mathrm{obs}}$ is the range of target centre wrt. the observer, i.e. Herschel, $\alpha$ is the Phase angle with indication of $\mathrm{L}(\mathrm{eading})$ or $\mathrm{T}(\mathrm{railing})$ the Sun, and $\theta_{\mathrm{U}-\mathrm{O}}$ is the angular separation from Uranus. $\dot{r}_{\text {helio }}$ is the heliocentric range rate and $\frac{\dot{r}_{\text {helio }}}{\left|\dot{r}_{\text {helio }}\right|}$ indicates $\mathrm{L}$ (eading) $\mathrm{H}(\mathrm{emisphere})$ or T(railing) $\mathrm{H}$ (emisphere), if the absolute value of the ratio is greater than $\frac{2}{3}$, or $\mathrm{B}$ (oth) $\mathrm{H}$ (emispheres) otherwise. All five figures have been computed with the JPL Horizons On-Line Ephemeris System. The five epochs with 4 observations each are separated by horizontal lines. 
Table A.6. PSF photometry of Miranda.

\begin{tabular}{|c|c|c|c|c|c|c|c|c|c|c|c|c|c|c|c|c|}
\hline OBSID & OD & $\begin{array}{c}\text { MJD } \\
\text { mid-time obs. }\end{array}$ & $\begin{array}{r}\lambda_{\mathrm{ref}} \\
\mu \mathrm{m}\end{array}$ & $\begin{array}{l}\frac{f_{\text {moon }}}{f_{\text {Uranus }}} \\
\left(10^{-3}\right)\end{array}$ & $\begin{array}{c}f_{\text {moon }} \\
(\mathrm{Jy})\end{array}$ & $\begin{array}{l}\sigma_{\mathrm{par}} \\
(\mathrm{Jy})\end{array}$ & $\begin{array}{l}\sigma_{\text {red }} \\
(\mathrm{Jy})\end{array}$ & $\begin{array}{l}\sigma_{\text {tot }} \\
(\mathrm{Jy})\end{array}$ & $\begin{array}{c}f_{\mathrm{moon}, \mathrm{cc}} \\
(\mathrm{Jy})\end{array}$ & $\begin{array}{c}f_{\text {model }} \\
(\mathrm{Jy})\end{array}$ & $\frac{f_{\text {moon,ce }}}{f_{\text {model }}}$ & $\begin{array}{l}r_{\text {helio }} \\
(\mathrm{AU})\end{array}$ & $\begin{array}{l}\Delta_{\mathrm{obs}} \\
(\mathrm{AU})\end{array}$ & $\begin{array}{c}\alpha \\
(\mathrm{deg})\end{array}$ & $\begin{array}{c}\theta_{\mathrm{U}-\mathrm{O}} \\
\left({ }^{\prime \prime}\right)\end{array}$ & $\frac{\dot{r}_{\text {helio }}}{\left|\dot{r}_{\text {helio }}^{\text {max }}\right|}$ \\
\hline 42211117 & 579 & 55543.72060 & 70.0 & 0.141 & 0.122 & 0.0996 & 0.0650 & 0.1189 & 0.124 & 0.129 & 0.964 & 20.089 & 19.974 & L 2.826 & 7.56 & $+0.84(\mathrm{TH})$ \\
\hline 2211118 & 579 & 55543.72464 & 70.0 & 0.299 & 0.258 & 0.1219 & 0.0253 & 0.1244 & 0.263 & 0.129 & 2.038 & 20.089 & 19.975 & L 2.826 & 7.65 & $+0.85(\mathrm{TH})$ \\
\hline 1342211120 & 579 & 55543.73128 & 100.0 & 0.110 & 0.097 & 0.2645 & 0.0254 & 0.2657 & 0.097 & 0.112 & 0.870 & 20.090 & 19.975 & L 2.826 & 7.78 & $+0.86(\mathrm{TH})$ \\
\hline 1342211121 & 579 & 55543.73532 & 100.0 & 0.187 & 0.165 & 0.2922 & 0.0810 & 0.3032 & 0.166 & 0.112 & 1.480 & 20.090 & 19.975 & L 2.826 & 7.85 & $+0.87(\mathrm{TH})$ \\
\hline 42223982 & 789 & 55754.05794 & 70.0 & 0.102 & 0.090 & 0.3742 & 0.0720 & 0.3810 & 0.092 & 0.134 & 0.684 & 20.082 & 19.801 & T 2.837 & 3.50 & $-0.21(\mathrm{BH})$ \\
\hline 1342223983 & 789 & 55754.06198 & 70.0 & 0.042 & 0.037 & 0.6195 & 0.0482 & 0.6213 & 0.037 & 0.134 & 0.279 & 20.082 & 19.800 & T 2.837 & 3.43 & $-0.19(\mathrm{BH})$ \\
\hline 1342223985 & 789 & 55754.06862 & 100.0 & - & - & - & - & - & - & 0.115 & - & 20.082 & 19.800 & Т 2.837 & 3.33 & $-0.17(\mathrm{BH})$ \\
\hline 1342223986 & 789 & 55754.07266 & 100.0 & - & - & - & - & - & - & 0.116 & - & 20.082 & 19.800 & T 2.837 & 3.27 & $-0.15(\mathrm{BH})$ \\
\hline 629 & 957 & 924 & 70.0 & 0.435 & 0.371 & 0.1109 & 0.0656 & 0.1289 & 0.378 & 0.128 & 2.954 & 20.077 & 20.118 & L 2.828 & 8.91 & $-1.00(\mathrm{LH})$ \\
\hline 2235630 & 957 & 55921.95328 & 70.0 & 0.228 & 0.194 & 0.0926 & 0.0744 & 0.1188 & 0.197 & 0.128 & 1.542 & 20.076 & 20.118 & L 2.828 & 8.91 & $-1.00(\mathrm{LH})$ \\
\hline 1342235632 & 957 & 55921.95992 & 100.0 & 0.158 & 0.137 & 0.2457 & 0.0765 & 0.2573 & 0.137 & 0.111 & 1.238 & 20.076 & 20.118 & L 2.828 & 8.91 & $-1.00(\mathrm{LH})$ \\
\hline 1342235633 & 957 & 55921.96396 & 100.0 & 0.098 & 0.085 & 0.2618 & 0.0242 & 0.2629 & 0.085 & 0.111 & 0.767 & 20.076 & 20.118 & L 2.828 & 8.91 & $-1.00(\mathrm{LH})$ \\
\hline 16772 & 1121 & & 70.0 & & U & 037 & 0 . & 98 & & & & 68 & & & 4.53 & -0.3 \\
\hline 1342246773 & 1121 & 56086.18 & 70.0 & 0. & 0. & 0.4240 & 0.0450 & 0.4264 & 0 & 0 & 1. & 20. & 20 & 45 & 4.44 & $\mathrm{BH})$ \\
\hline 1342246774 & 1121 & 5608 & 100.0 & & & 0.6144 & 0.0148 & 0.6 & & 10 & 4 & 20 & & & 4.35 & $\mathrm{BH})$ \\
\hline 1342246775 & 1121 & 56086.19712 & 100.0 & 0.160 & 0.135 & 0.5923 & 0.0373 & 0.5934 & 0.135 & 0.110 & 1.231 & 20.068 & 20.404 & T 2.745 & 4.26 & $-0.34(\mathrm{BH})$ \\
\hline 1342257193 & 1310 & $8 / 501$ & 70.0 & 0.329 & 0.289 & 2983 & 0.0739 & 0.3073 & 0.294 & 0.134 & 2.193 & 20.059 & 19.823 & L 2.773 & 4.74 & $-0.46(\mathrm{BH})$ \\
\hline 1342257194 & 1310 & 56275.07765 & 70.0 & 0.191 & 0.167 & 0.2981 & 0.0610 & 0.3042 & 0.170 & 0.134 & 1.266 & 20.059 & 19.823 & L 2.773 & 4.85 & $-0.48(\mathrm{BH})$ \\
\hline 1342257195 & 1310 & 56275.08169 & 100.0 & 0.048 & 0.044 & 0.4470 & 0.0228 & 0.4476 & 0.044 & 0.116 & 0.376 & 20.059 & 19.823 & L 2.773 & 4.97 & $-0.49(\mathrm{BH})$ \\
\hline 1342257196 & 1310 & 56275.08573 & 100.0 & 0.170 & 0.152 & 0.4948 & 0.0481 & 0.4971 & 0.153 & 0.116 & 1.315 & 20.059 & 19.823 & L 2.773 & 5.08 & $-0.51(\mathrm{BH})$ \\
\hline
\end{tabular}

Notes. Applied colour correction factors cc to derive colour corrected fluxes $f_{\text {moon,cc }}$, are listed in Table 7. $r_{\text {helio }}$ is the light-time corrected heliocentric range, $\Delta_{\mathrm{obs}}$ is the range of target centre wrt. the observer, i.e. Herschel, $\alpha$ is the Phase angle with indication of $\mathrm{L}(\mathrm{eading})$ or $\mathrm{T}(\mathrm{railing})$ the Sun, and $\theta_{\mathrm{U}-\mathrm{O}}$ is the angular separation from Uranus. $\dot{r}_{\text {helio }}$ is the heliocentric range rate and $\frac{\dot{r}_{\text {helio }}}{\left|\dot{r}_{\text {heli }}\right|}$ indicates L(eading) $\mathrm{H}(\mathrm{emisphere})$ or T(railing) $\mathrm{H}$ (emisphere), if the absolute value of the ratio is greater than $\frac{2}{3}$, or $\mathrm{B}$ (oth) $\mathrm{H}$ (emispheres) otherwise. All five figures have been computed with the JPL Horizons On-Line Ephemeris System.

\section{A.6. Comparison of PSF photometry with aperture photometry for selected measurements}

Table A.7. Aperture photometry of Uranian moons for selected measurements when the moon image was well separated from Uranus (minimum aperture edge distance from Uranus was 8.'77, 10.'79, and 16.'57 at 70, 100, and $160 \mu \mathrm{m}$, respectively) and any PSF residuals and comparison with the corresponding PSF photometry.

\begin{tabular}{|c|c|c|c|c|c|c|c|c|c|c|c|}
\hline OBSID & OD & $\begin{array}{c}\text { MJD } \\
\text { mid-time obs. }\end{array}$ & Object & $\begin{array}{l}\lambda_{\text {ref }} \\
\mu \mathrm{m}\end{array}$ & $\begin{array}{c}r^{\text {aperture }} \\
\left({ }^{\prime \prime}\right)\end{array}$ & $\begin{array}{c}f_{\text {moon }}^{\text {aperture }} \\
(\mathrm{Jy})\end{array}$ & $\begin{array}{c}\sigma_{\text {aper }} \\
(\mathrm{Jy})\end{array}$ & $\begin{array}{c}f_{\text {moon }}^{\mathrm{PSF}} \\
(\mathrm{Jy})\end{array}$ & $\begin{array}{l}\sigma_{\text {tot }} \\
(\mathrm{Jy})\end{array}$ & $\frac{f^{\mathrm{PSF}}}{f^{\text {aper }}}$ & $\sigma_{\text {ratio }}$ \\
\hline \multirow[t]{3}{*}{1342211117} & 579 & 55543.72060 & Titania & 70.0 & 5.6 & 1.635 & 0.012 & 1.669 & 0.017 & 1.021 & 0.013 \\
\hline & & & & 160.0 & 10.7 & 0.827 & 0.030 & 0.875 & 0.011 & 1.058 & 0.041 \\
\hline & & & Umbriel & 70.0 & 5.6 & 0.807 & 0.017 & 0.833 & 0.019 & 1.032 & 0.032 \\
\hline \multirow[t]{3}{*}{1342211118} & 579 & 55543.72464 & Titania & 70.0 & 5.6 & 1.622 & 0.012 & 1.680 & 0.018 & 1.036 & 0.013 \\
\hline & & & & 160.0 & 10.7 & 0.833 & 0.029 & 0.856 & 0.016 & 1.028 & 0.041 \\
\hline & & & Umbriel & 70.0 & 5.6 & 0.844 & 0.017 & 0.880 & 0.012 & 1.043 & 0.025 \\
\hline \multirow[t]{3}{*}{1342211120} & 579 & 55543.73128 & Titania & 100.0 & 6.8 & 1.397 & 0.009 & 1.412 & 0.006 & 1.011 & 0.008 \\
\hline & & & & 160.0 & 10.7 & 0.813 & 0.045 & 0.864 & 0.010 & 1.063 & 0.060 \\
\hline & & & Umbriel & 100.0 & 6.8 & 0.592 & 0.043 & 0.718 & 0.010 & 1.213 & 0.090 \\
\hline \multirow[t]{3}{*}{1342211121} & 579 & 55543.73532 & Titania & 100.0 & 6.8 & 1.370 & 0.013 & 1.398 & 0.006 & 1.020 & 0.011 \\
\hline & & & & 160.0 & 10.7 & 0.838 & 0.031 & 0.854 & 0.014 & 1.019 & 0.041 \\
\hline & & & Umbriel & 100.0 & 6.8 & 0.704 & 0.028 & 0.762 & 0.008 & 1.082 & 0.045 \\
\hline \multirow[t]{5}{*}{1342223982} & 789 & 55754.05794 & Titania & 70.0 & 5.6 & 1.655 & 0.025 & 1.705 & 0.015 & 1.030 & 0.018 \\
\hline & & & & 160.0 & 10.7 & 0.838 & 0.012 & 0.884 & 0.009 & 1.055 & 0.019 \\
\hline & & & Oberon & 70.0 & 5.6 & 1.549 & 0.017 & 1.611 & 0.008 & 1.040 & 0.013 \\
\hline & & & & 160.0 & 10.7 & 0.779 & 0.016 & 0.807 & 0.008 & 1.036 & 0.024 \\
\hline & & & Arie & 70.0 & 5.6 & 0.833 & 0.045 & 0.821 & 0.012 & 0.986 & 0.055 \\
\hline \multirow[t]{2}{*}{1342223983} & 789 & 55754.06198 & Titania & 70.0 & 5.6 & 1.641 & 0.023 & 1.710 & 0.016 & 1.042 & 0.018 \\
\hline & & & & 160.0 & 10.7 & 0.869 & 0.011 & 0.907 & 0.007 & 1.044 & 0.015 \\
\hline
\end{tabular}

Notes. The aperture photometry has been corrected for the finite aperture size according to the description in the PACS Handbook (Exter et al. 2018), Sect. 7.5.2. 
Table A.7. continued.

\begin{tabular}{|c|c|c|c|c|c|c|c|c|c|c|c|}
\hline OBSID & OD & $\begin{array}{c}\text { MJD } \\
\text { mid-time obs. }\end{array}$ & Object & $\begin{array}{l}\lambda_{\text {ref }} \\
\mu \mathrm{m}\end{array}$ & $\begin{array}{c}r^{\text {aperture }} \\
\left({ }^{\prime \prime}\right)\end{array}$ & $\begin{array}{c}f_{\text {moon }}^{\text {aperture }} \\
(\mathrm{Jy})\end{array}$ & $\begin{array}{c}\sigma_{\text {aper }} \\
(\mathrm{Jy})\end{array}$ & $\begin{array}{c}f_{\text {moon }}^{\mathrm{PSF}} \\
(\mathrm{Jy})\end{array}$ & $\begin{array}{l}\sigma_{\text {tot }} \\
(\mathrm{Jy})\end{array}$ & $\frac{f^{\mathrm{PSF}}}{f^{\mathrm{aper}}}$ & $\sigma_{\text {ratio }}$ \\
\hline & & & Oberon & 70.0 & 5.6 & 1.581 & 0.014 & 1.638 & 0.012 & 1.036 & 0.012 \\
\hline & & & & 160.0 & 10.7 & 0.836 & 0.014 & 0.836 & 0.012 & 1.000 & 0.022 \\
\hline & & & Ariel & 70.0 & 5.6 & 0.681 & 0.061 & 0.798 & 0.023 & 1.172 & 0.110 \\
\hline \multirow[t]{4}{*}{1342223985} & 789 & 55754.06862 & Titania & 100.0 & 6.8 & 1.418 & 0.010 & 1.455 & 0.005 & 1.026 & 0.008 \\
\hline & & & & 160.0 & 10.7 & 0.862 & 0.015 & 0.868 & 0.016 & 1.007 & 0.026 \\
\hline & & & Oberon & 100.0 & 6.8 & 1.331 & 0.011 & 1.374 & 0.012 & 1.032 & 0.012 \\
\hline & & & & 160.0 & 10.7 & 0.810 & 0.011 & 0.826 & 0.005 & 1.020 & 0.015 \\
\hline \multirow[t]{4}{*}{1342223986} & 789 & 55754.07266 & Titania & 100.0 & 6.8 & 1.434 & 0.013 & 1.455 & 0.005 & 1.015 & 0.010 \\
\hline & & & & 160.0 & 10.7 & 0.833 & 0.015 & 0.883 & 0.009 & 1.060 & 0.022 \\
\hline & & & Oberon & 100.0 & 6.8 & 1.347 & 0.016 & 1.398 & 0.013 & 1.038 & 0.016 \\
\hline & & & & 160.0 & 10.7 & 0.838 & 0.015 & 0.839 & 0.008 & 1.001 & 0.020 \\
\hline \multirow[t]{4}{*}{1342235629} & 957 & 55921.94924 & Titania & 70.0 & 5.6 & 1.592 & 0.044 & 1.630 & 0.097 & 1.024 & 0.067 \\
\hline & & & Oberon & 70.0 & 5.6 & 1.557 & 0.013 & 1.573 & 0.014 & 1.010 & 0.012 \\
\hline & & & & 160.0 & 10.7 & 0.785 & 0.012 & 0.819 & 0.011 & 1.043 & 0.021 \\
\hline & & & Ariel & 70.0 & 5.6 & 0.644 & 0.129 & 0.726 & 0.034 & 1.127 & 0.232 \\
\hline \multirow[t]{4}{*}{1342235630} & 957 & 55921.95328 & Titania & 70.0 & 5.6 & 1.550 & 0.041 & 1.684 & 0.021 & 1.086 & 0.032 \\
\hline & & & Oberon & 70.0 & 5.6 & 1.556 & 0.023 & 1.576 & 0.015 & 1.013 & 0.018 \\
\hline & & & & 160.0 & 10.7 & 0.758 & 0.011 & 0.800 & 0.006 & 1.055 & 0.017 \\
\hline & & & Ariel & 70.0 & 5.6 & 0.651 & 0.062 & 0.702 & 0.017 & 1.078 & 0.106 \\
\hline \multirow[t]{3}{*}{1342235632} & 957 & 55921.95992 & Titania & 100.0 & 6.8 & 1.388 & 0.019 & 1.449 & 0.008 & 1.083 & 0.016 \\
\hline & & & Oberon & 100.0 & 6.8 & 1.296 & 0.012 & 1.320 & 0.009 & 1.019 & 0.012 \\
\hline & & & & 160.0 & 10.7 & 0.762 & 0.011 & 0.814 & 0.009 & 1.068 & 0.019 \\
\hline \multirow[t]{3}{*}{1342235633} & 957 & 55921.96396 & Titania & 100.0 & 6.8 & 1.398 & 0.040 & 1.466 & 0.011 & 1.049 & 0.031 \\
\hline & & & Oberon & 100.0 & 6.8 & 1.306 & 0.019 & 1.353 & 0.009 & 1.036 & 0.017 \\
\hline & & & & 160.0 & 10.7 & 0.785 & 0.019 & 0.819 & 0.013 & 1.043 & 0.030 \\
\hline \multirow[t]{3}{*}{1342246772} & 1121 & 56086.18500 & Titania & 70.0 & 5.6 & 1.554 & 0.017 & 1.573 & 0.012 & 1.012 & 0.013 \\
\hline & & & Oberon & 70.0 & 5.6 & 1.558 & 0.028 & 1.597 & 0.034 & 1.025 & 0.029 \\
\hline & & & & 160.0 & 10.7 & 0.768 & 0.014 & 0.792 & 0.010 & 1.031 & 0.023 \\
\hline \multirow[t]{3}{*}{1342246773} & 1121 & 56086.18904 & Titania & 70.0 & 5.6 & 1.581 & 0.030 & 1.630 & 0.038 & 1.031 & 0.031 \\
\hline & & & Oberon & 70.0 & 5.6 & 1.517 & 0.019 & 1.566 & 0.033 & 1.032 & 0.025 \\
\hline & & & & 160.0 & 10.7 & 0.768 & 0.007 & 0.776 & 0.009 & 1.010 & 0.015 \\
\hline \multirow[t]{3}{*}{1342246774} & 1121 & 56086.19308 & Titania & 100.0 & 6.8 & 1.283 & 0.011 & 1.318 & 0.010 & 1.027 & 0.012 \\
\hline & & & Oberon & 100.0 & 6.8 & 1.273 & 0.014 & 1.300 & 0.006 & 1.021 & 0.012 \\
\hline & & & & 160.0 & 10.7 & 0.751 & 0.018 & 0.781 & 0.005 & 1.040 & 0.026 \\
\hline \multirow[t]{3}{*}{1342246775} & 1121 & 56086.19712 & Titania & 100.0 & 6.8 & 1.316 & 0.028 & 1.343 & 0.008 & 1.021 & 0.023 \\
\hline & & & Oberon & 100.0 & 6.8 & 1.242 & 0.008 & 1.289 & 0.008 & 1.038 & 0.009 \\
\hline & & & & 160.0 & 10.7 & 0.739 & 0.011 & 0.762 & 0.008 & 1.031 & 0.019 \\
\hline \multirow[t]{5}{*}{1342257193} & 1310 & 56275.07361 & Titania & 70.0 & 5.6 & 1.681 & 0.016 & 1.724 & 0.018 & 1.026 & 0.014 \\
\hline & & & & 160.0 & 10.7 & 0.894 & 0.027 & 0.931 & 0.017 & 1.041 & 0.037 \\
\hline & & & Oberon & 70.0 & 5.6 & 1.575 & 0.012 & 1.625 & 0.013 & 1.032 & 0.011 \\
\hline & & & & 160.0 & 10.7 & 0.791 & 0.011 & 0.811 & 0.006 & 1.025 & 0.016 \\
\hline & & & Umbriel & 70.0 & 5.6 & 0.922 & 0.017 & 0.947 & 0.008 & 1.027 & 0.021 \\
\hline \multirow[t]{5}{*}{1342257194} & 1310 & 56275.07765 & Titania & 70.0 & 5.6 & 1.656 & 0.019 & 1.729 & 0.014 & 1.044 & 0.015 \\
\hline & & & & 160.0 & 10.7 & 0.916 & 0.019 & 0.933 & 0.017 & 1.019 & 0.028 \\
\hline & & & Oberon & 70.0 & 5.6 & 1.556 & 0.022 & 1.632 & 0.018 & 1.049 & 0.019 \\
\hline & & & & 160.0 & 10.7 & 0.772 & 0.008 & 0.816 & 0.005 & 1.057 & 0.013 \\
\hline & & & Umbriel & 70.0 & 5.6 & 0.920 & 0.012 & 0.964 & 0.011 & 1.048 & 0.018 \\
\hline \multirow[t]{5}{*}{1342257195} & 1310 & 56275.08169 & Titania & 100.0 & 6.8 & 1.449 & 0.013 & 1.487 & 0.015 & 1.026 & 0.014 \\
\hline & & & & 160.0 & 10.7 & 0.915 & 0.037 & 0.955 & 0.025 & 1.044 & 0.050 \\
\hline & & & Oberon & 100.0 & 6.8 & 1.326 & 0.017 & 1.396 & 0.015 & 1.053 & 0.018 \\
\hline & & & & 160.0 & 10.7 & 0.797 & 0.012 & 0.828 & 0.005 & 1.039 & 0.017 \\
\hline & & & Umbriel & 100.0 & 6.8 & 0.736 & 0.026 & 0.814 & 0.008 & 1.106 & 0.041 \\
\hline \multirow{5}{*}{1342257196} & 1310 & 56275.08573 & Titania & 100.0 & 6.8 & 1.420 & 0.014 & 1.470 & 0.005 & 1.035 & 0.011 \\
\hline & & & & 160.0 & 10.7 & 0.958 & 0.031 & 0.952 & 0.027 & 0.994 & 0.043 \\
\hline & & & Oberon & 100.0 & 6.8 & 1.324 & 0.008 & 1.382 & 0.013 & 1.044 & 0.012 \\
\hline & & & & 160.0 & 10.7 & 0.766 & 0.013 & 0.799 & 0.010 & 1.043 & 0.022 \\
\hline & & & Umbriel & 100.0 & 6.8 & 0.703 & 0.009 & 0.802 & 0.007 & 1.141 & 0.018 \\
\hline
\end{tabular}




\section{Appendix B: Relation of PACS photometer detector response with the telescope background power in the 70,100 , and $160 \mu \mathrm{m}$ PACS filters}

Table B.1. Telescope background correction factors for the Uranus observations derived from Eqs. (B.3)-( B.5).

\begin{tabular}{rcccc}
\hline \hline OD & $c_{\text {telbg }}^{70}$ & $c_{\text {telbg }}^{100}$ & $c_{\text {telbg }}^{160}$ & $c_{\text {dist }}$ \\
\hline 579 & 1.0001 & 0.9989 & 1.0009 & 1.0046 \\
789 & 1.0020 & 1.0007 & 1.0038 & 1.0223 \\
957 & 0.9939 & 0.9965 & 0.9979 & 0.9903 \\
1121 & 0.9976 & 0.9990 & 1.0020 & 0.9627 \\
1310 & 0.9892 & 0.9947 & 0.9959 & 1.0201 \\
\hline
\end{tabular}

Notes. The last column gives the distance correction (dc) factor to bring the Uranus photometry to a mean distance.

According to Exter et al. (2018), Sect. 7.4.2, the monochromatic PACS flux density is inversely proportional to the detector response $R$ :

$f_{v, 1}\left(\lambda_{0}\right)[\mathrm{Jy}]=U_{\text {sig }} \frac{C_{\text {conv }}}{R}=\frac{U_{\text {sig }}}{R_{v, 1}}$

with

$R_{v, 1}[\mathrm{~V} / \mathrm{Jy}]=\frac{R[\mathrm{~V} / \mathrm{W}]}{C_{\text {conv }}[\mathrm{Jy} / \mathrm{pW}]}$.

$R_{v, 1}$ is actually not a constant. It depends on the operational temperature of the bolometers and the IR total flux load, hence $R_{v, 1}=f\left(T, B_{\text {totalflux }}\right)$. $B_{\text {totalflux }}$ is dominated by the background of the only passively cooled telescope $B_{\text {telescope }}$. A first description of this detector response effect by the telescope background was given by Balog et al. (2014). In that study the telescope background was described as flux/per spectrometer pixel. Klaas (2016) describes a telescope background model, from which a telescope background per photometer pixel can be calculated for each Herschel Operational Day (OD). In Sect. 6 there detector response relations with regard to this calculated telescope background are shown which are based on observations of standard stars. In particular at $160 \mu \mathrm{m}$ the stars are already quite faint $(<3 \mathrm{Jy})$ and no significant correlation could be derived due to the uncertainties of the measured fluxes and hence a large scatter of the data points.

However, the Uranus observations offer high $\mathrm{S} / \mathrm{N}$ data points for all three filters. The only prerequisite is to scale all observations to the same distance $(\mathrm{dc})$. Figure B.1 shows the derived relations for the correction factors $c_{\text {telbg }}\left(B_{\text {telescope }}\right)=$ $\left.\left(\frac{f_{\text {meas }}^{\text {Uranus }}\left(B_{\text {telescope }}\right)}{f_{\text {model }}^{\text {Uranus }}}\right)\right)_{\mathrm{dc}}$. These are (from PSF photometry):

$$
\begin{aligned}
& 70 \mu \mathrm{m}: c_{\text {telbg }}=1.2445-0.1041 \times B_{\text {telescope }}(p W) \\
& 100 \mu \mathrm{m}: c_{\text {telbg }}=1.1859-0.1496 \times B_{\text {telescope }}(p W) \\
& 160 \mu \mathrm{m}: c_{\text {telbg }}=1.3678-0.2015 \times B_{\text {telescope }}(p W)
\end{aligned}
$$

For our Uranus observations the following correction factors in Table B.1 are applied to the fluxes (by division, since $\mathrm{R}$ is inversely proportional with $\mathrm{f}_{v, 1}\left(\lambda_{0}\right)$ ).

While the effect of the distance correction $\left(\frac{c_{\text {dist }}^{\max }}{c_{\text {dist }}^{\text {min }}}\right)$ is in the order of $6 \%$, the effect of the detector response change with
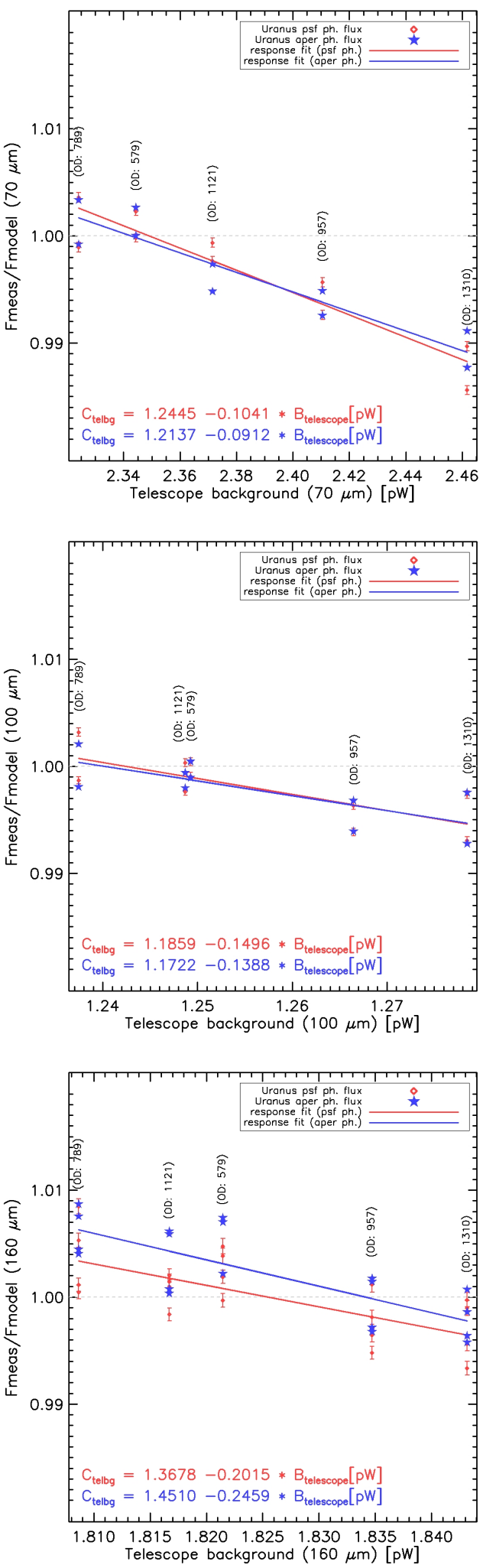

Fig. B.1. Relation of PACS photometer detector response, as indicated by a normalised flux level, with the telescope background power for the 70,100 , and $160 \mu \mathrm{m}$ filters. The fits were done both for PSF photometry (red) and aperture photometry (blue).

telescope background $\left(\frac{c_{\text {telbg }}^{\max }}{c_{\text {telbg }}^{\min }}\right)$ is in the order of $1.3 \%, 0.6 \%$, and $0.8 \%$ at 70,100 , and $160 \mu \mathrm{m}$, respectively, for the data set of Uranus and its satellites. 